NASA TECHNICAL REPORT

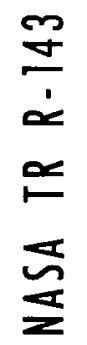

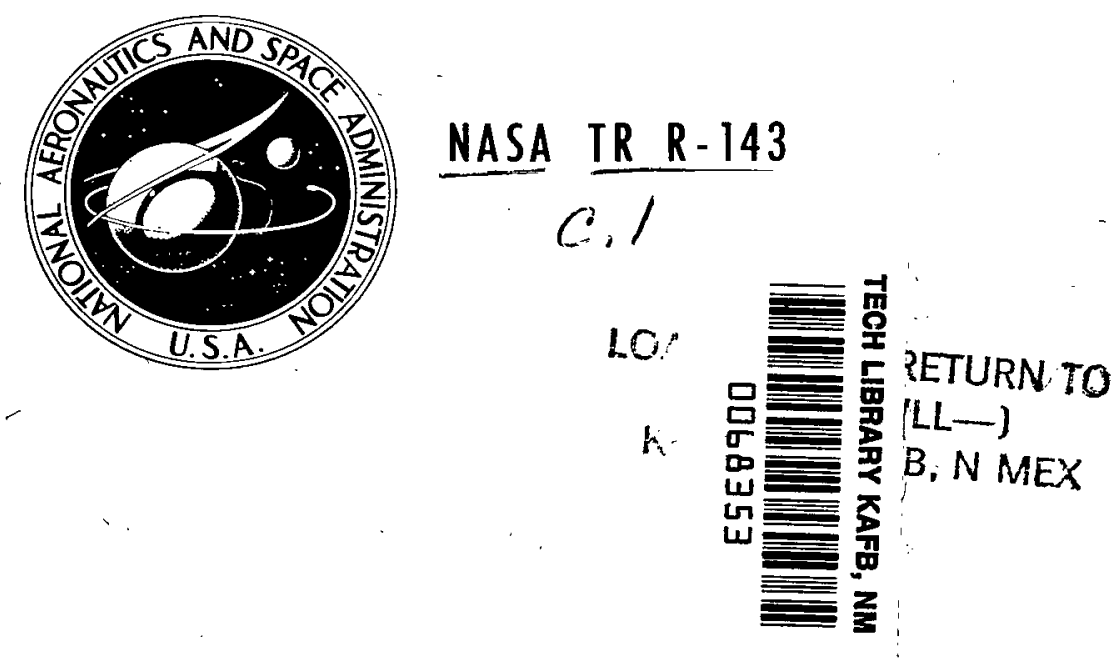

SPECTRUM OF HYDROMAGNETIC WAVES

IN THE EXOSPHERE

by Gordon J. F. MacDonald

Goddard'Space Flight Center

Greenbelt, Maryland

national aeronautics and SPACE administration - Washington, D. C. DECEMber 1962 


\section{SPECTRUM OF}

\section{HYDROMAGNETIC WAVES IN THE EXOSPHERE}

By Gordon J. F. MacDonald

Goddard Space Flight Center

Greenbelt, Maryland

NATIONAL AERONAUTICS AND SPACE ADMINISTRATION

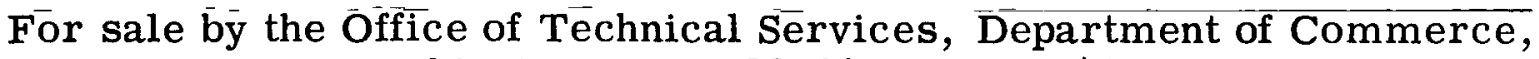
Washington, D.C. 20230 -- Price $\$ 1.75$ 


\title{
SPECTRUM OF \\ HYDROMAGNETIC WAVES IN THE EXOSPHERE
}

\author{
by \\ Gordon J. F. MacDonald \\ Goddard Space Flight Center
}

\section{SUMMARY}

\begin{abstract}
A disturbance in the exosphere generates waves in three partially separable modes. These modes are described by considering the vorticity about a line of force, the two-dimensional divergence of velocity in the plane perpendicular to the line of force, and the component of velocity along the line of force. The propagation of vorticity is one-dimensional and there is no geometrical attenuation; energy is lost only through the finite conductivity of the medium. The propagation of the longitudinal velocity component is almost one-dimensional but is heavily damped at high frequencies. In a gravitational field, the medium is no longer uniform and at low frequencies the modes are coupled in a complicated way. For parallel magnetic and gravitational fields, the vorticity mode is still separable and gravity leads to anisotropic dispersion in the other modes.
\end{abstract}




\section{CONTENTS}

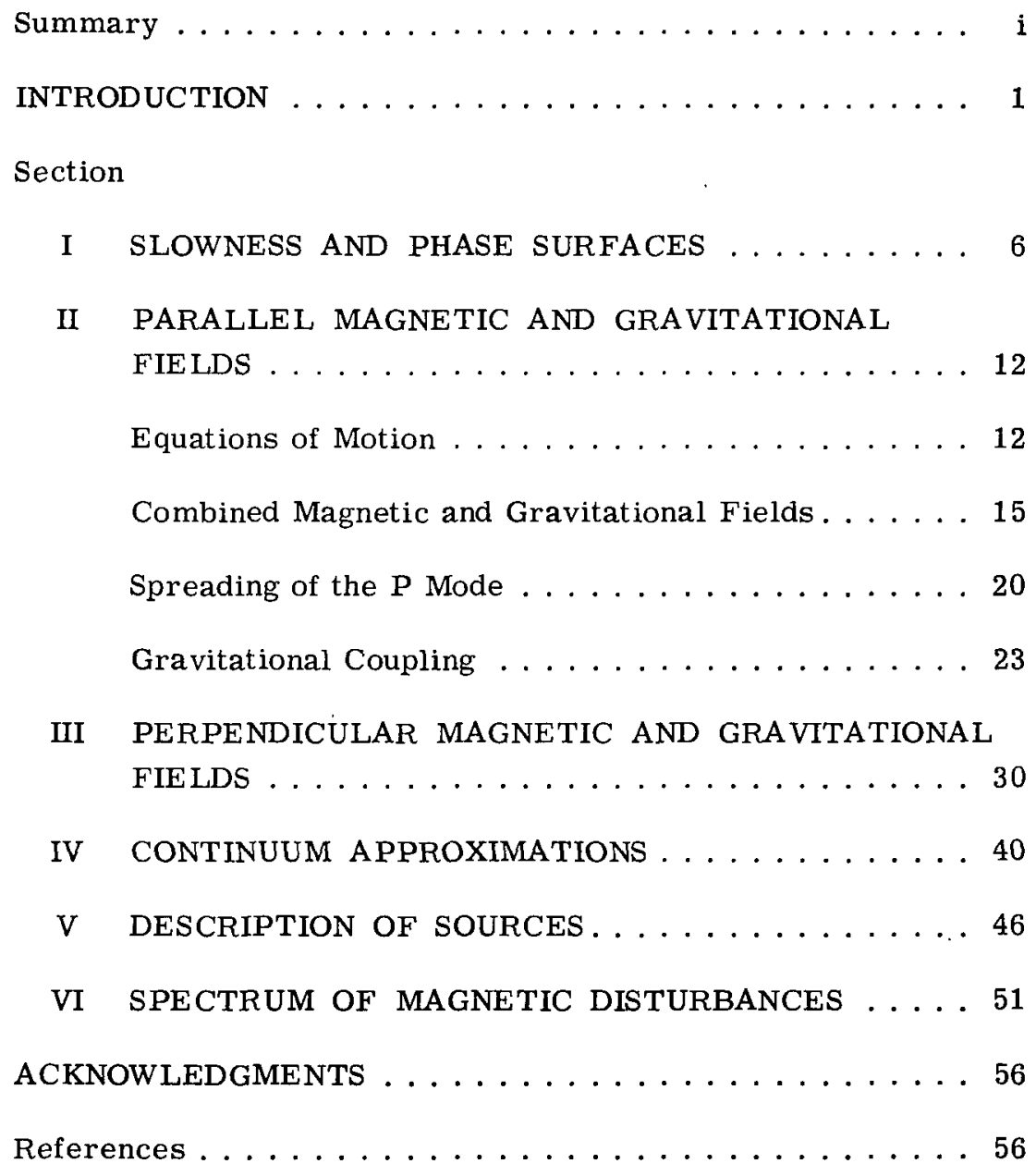




\title{
SPECTRUM OF \\ HYDROMAGNETIC WAVES IN THE EXOSPHERE*
}

\author{
by \\ Gordon J. F. MacDonald $\uparrow$ \\ Goddard Space Flight Center
}

\section{INTRODUCTION}

The theory of magnetic storms has undergone a remarkable development in the past few years. As early as 1896 Birkeland proposed that magnetic storms were the result of streams of particles emitted from the sun and guided towards the earth by the earth's magnetic field. Chapman and Ferraro (References 1, 2, and 3) elabnrated on this solar particle theory, which presumes that the space between the earth and the sun is a vacuum and that the induced currents in the plasma are propagated with the speed of light.

Investigations of whistlers by Storey (Reference 4) and of zodiacal light by Behr and Siedentopf (Reference 5) indicated that interplanetary space contains a resident ionized gas with a density of a few hundred electrons $/ \mathrm{cm}^{3}$. A recent investigation of the zodiacal light by Blackwell and Ingham (Reference 6) limits the density to less than 120 electrons $/ \mathrm{cm}^{3}$ in the outer corona, while further whistler studies suggest a comparable value for the upper regions of the exosphere. The presence of a resident interplanetary ionized gas requires that the propagation of a solar stream into the earth's field be treated as a problem in hydromagnetics (Reference 7). Dessler and Parker (Reference 8) have undertaken a detailed investigation of a hydromagnetic theory of geomagnetic storms [an alternative development is due to Piddington (References 9, 10, and 11)]: The magnetic storm results from fluctuations in the intensity of particles streaming from the sun, and disturbances are propagated through the exosphere as hydromagnetic waves. On reaching the base of the ionosphere, the hydromagnetic signals are transmitted through the atmosphere as ordinary electromagnetic waves at the speed of light. In the lower ionosphere, with its high concentration of neutral particles, the propagation is complicated and may take the form of a diffusing electromagnetic wave. The hydromagnetic

*Also published in J. Geophys. Res. 66(11):3639-3670, November 1961.

†Now at the In'stitute of Geophysics and Planetary Physics, University of California. 
theory of geomagnetic storms thus proposes that the magnetic signal is transferred through the exosphere at the local Alfvén wave velocity and through the un-ionized atmosphere at the speed of light.

Dessler and Parker, in their development of the hydromagnetic theory, emphasize the characteristic times associated with various events in a magnetic storm. Dessler, Francis and Parker (Reference 12) show that the time taken for hydromagnetic waves to pass from the solar plasma front to ground level is on the order of a few minutes. This prediction is in accord with the observed rise time of a sudden commencement. [The simultaneity of the world-wide increase in intensity of the horizontal component is open to question because varying standards of timing are used by geomagnetic observatories. Williams (Reference 13) finds that sudden commencements are registered first in the high and middle latitudes.]

While much progress has been made in determining the time characteristics of geomagnetic storms, less effort has been expended in studying the frequency characteristics of geomagnetic variations. A number of peridiogram, or zero-crossing, analyses have been made on geomagnetic records. Because of the noise level and the complicated spectra, neither method can lead to reliable spectral estimates. Perhaps the principal reason for the lack of spectral estimates is the analog recording usually used with magnetometers and earth current coils, and the difficulty in converting an analog record to a digital one.

The sharp nature of the onset of a magnetic storm indicates a spectrum relatively rich at high (cycles per second to cycles per minute) frequencies. The spectrum associated with the sharp onset may contain evidence of the source. Dessler and Parker (Reference 8) suggest that there is a high frequency cutoff fixed by the Larmor frequency at the source. The long time scale associated with the main phase of the magnetic storm suggests that the spectrum will also contain energy at much lower (cycles per hour) frequencies. Furthermore, instruments of sufficient sensitivity establish the existence of micropulsations in the geomagnetic field. These are commonly associated with the onset of magnetic storms, and show sinusoidal time variations with periods ranging from a few tenths of a second to a hundred seconds (References 14, 15, and 16). It is tempting to infer, from the narrow band of frequencies associated with the low-frequency micropulsations, that they represent a resonant mode of the exosphere. This interpretation is strengthened by their presence during periods of relative magnetic quiet.

The present study is an attempt to provide a partial background for the separation of source effects from those of the medium as a prelude to the spectral analysis of magnetic records. A major limitation is the neglect of all dissipation. The techniques and methodology have a close resemblance to those of acoustics and seismology. 
In the presence of a magnetic field a disturbance is propagated through a uniform, electrically conducting fluid by three wave fronts traveling at three different speeds (References 17-30). These three modes are illustrated by the base in which the Alfven wave velocity is large compared to the velocity of sound: The vorticity about the lines of force is propagated one-dimensionally at the Alfven wave velocity; the velocity component along the line of force is propagated ai nearly the velocity of sound and the propagation is almost one-dimensional; the two-dimensional divergence in the plane perpendicular to the lines of force, together with the longitudinal component of the magnetic field, is propagated almost spherically at the Alfvén wave velocity. For convenience we will label the one-dimensional vorticity mode as the $\mathrm{V}$ mode. The nearly one-dimensional sound wave is termed the pressure or P mode, while the spherically spreading mode is termed the transverse or $\mathbf{T}$ mode. If the Alfvén wave velocity is much less than the velocity of sound, then the $\mathrm{V}$ mode remains one-dimensional, the $\mathrm{P}$ mode is spherically spreading, and $\mathrm{T}$ mode is nearly one-dimensional. It should be emphasized that, in general, the solutions of the equations cannot be resolved into three modes characterized by a velocity, and that the wave fronts may interact to give a complicated waveform.

Part I of this paper presents a review of the theory of the propagation of waves in an anisotropic inhomogeneous medium; dissipation is ignored. The dynamical equations lead to dispersion relations which, for a fixed frequency, determine slowness surfaces in wave number space. The slowness surface, in turn, fixes the geometry of the propagating wave. The concept of group velocity is particularly important, since the direction of propagation of the phase of the wave need not coincide with the direction of the energy propagation. The J.W.K.B. approximation in the propagation of waves in an inhomogeneous medium is considered, and the limitations of the method are stressed. The method yields results which are valid only if the wavelength of the disturbance is short compared with the scale length of the variable properties. For many problems in hydromagnetic wave propagation in the exosphere this holds true, but the approximation is not as good for the interesting low frequency waves. Despite the limitations of the approximation, a qualitative picture of the low frequency waves may be obtained.

Parts II and III treat the propagation of the low frequency hydromagnetic waves with the aid of the diagnostic diagrams introduced by Eckart (Reference 21) in his study of classical gravity waves. Such a diagnostic diagram is a projection, in the wave numberfrequency space, of the regions of allowed propagation modes. At low frequencies it is possible for gravity to modify the various hydromagnetic modes. In Part II, the case of the parallel magnetic and gravitational fields is considered, and the slowness surfaces and surfaces of constant phase are determined for various frequencies. Gravity leads to anisotropic dispersion; but its influence is negligible at high frequencies (periods much less than several minutes). 
There is a balance of particle and magnetic pressure in the $\mathrm{P}$ mode propagation: As the particle pressure decreases, suction is created and a magnetic field develops. In other words, an increase in the magnetic field is accompanied by a transfer of energy from the acoustic field, and the particle pressure decreases. The ratio of the variation in particle pressure to the variation in magnetic pressure is determined by the square of the ratio of the velocity of the sound wave to the Alfven wave velocity in the $T$ mode. The variation of particle pressure during the passage of a $T$ wave in the exosphere is small, while the $\mathrm{V}$ wave does not alter the thermodynamic state of the medium at all.

Part III considers the case of perpendicular gravitational and magnetic fields. The propagation is not cylindrically symmetrical if the lines of force are at an arbitrary angle to the direction of gravity. A detailed investigation of the influence of the gravitational field on hydromagnetic wave propagation is carried out in the case where the sound wave velocity is small compared to the Alfvén wave velocity, and this is used as an introduction to the general case.

The equations of hydromagnetics are continuum equations and imply that the constituent particles collectively interact. The limiting conditions in the exosphere are considered in Part IV. The generation of macroscopic hydromagnetic waves depends on whether the disturbances have a length greater than some characteristic length of the medium. For the propagation of $\mathrm{V}$ and $\mathrm{T}$ waves, this characteristic length is the Larmor radius of the positive ions. If the disturbance has a characteristic length that is short compared to the Larmor radius but small compared to the mean free path, then collisionless hydromagnetic waves are propagated. The critical length involved in the propagation of the $\mathrm{P}$ mode is the distance a particle must travel before exchanging an appreciable fraction of its momentum. Because of the long-range nature of the Coulomb forces, the particle may traverse short distances compared to the free path and still exchange a substantial portion of its momentum. For representative models of the exosphere, disturbances at distances of several earth radii must have length scales that are large compared to several kilometers in order that acoustic waves be propagated. The $\mathrm{V}$ and $\mathrm{T}$ waves are propagated if the length scale is large compared to some hundreds of meters in the outer regions of the exosphere.

The characterization of sources is taken up in Part V. The V mode is represented by a line in the diagnostic diagram, the $\mathrm{T}$ mode by a region. The $\mathrm{V}$ mode should be char acterized by a narrow spectrum, the $\mathrm{T}$ mode by a much broader spectrum. The $\mathrm{V}$ mode, of course, is generated only if the source produces vorticity; examples of such sources include the exhaust of a rocket motor, the wake of a satellite in the lower exosphere, and the turbulent region in the vicinity of a finite amplitude wave. The $\mathbf{P}$ and $\mathbf{T}$ modes are generated by pressure pulses; a typical source might be the influx of ionizing radiation into the upper ionosphere, giving a pressure pulse of charged particle gas. Such a pressure pulse would generate primarily spherically spreading $\mathrm{T}$ waves and one-dimensional 
acoustic waves; but if the discontinuity were sharp enough, vorticity might result. The proportion of $T$ and $P$ waves generated depends on a comparison of the characteristic times and length scales of the source with the regularity and wavelength of allowed waves. In the exosphere, waves of a given frequency will be predominantly $P$ waves if the source has a small length scale (meters), and $\mathrm{T}$ waves if the source has a larger length scale (kilometers). A satellite traveling in the lower exosphere will generate $\mathbf{P}$ waves and possibly $\mathrm{V}$ waves, but will not excite the $\mathrm{T}$ mode.

Part VI treats the free oscillations of the separable V mode. These are analogous to the oscillations of a weighted spring, and have periods dependent on the geomagnetic latitude of the line of force. Their free periods are comparable to the periods observed in the low frequency micropulsations. The pearl type of micropulsation is interpreted as the excitation of a $\mathrm{V}$ mode with a narrow spectrum. The time representation of this narrow band is then a modulated sine wave characteristic of the pearl micropulsation. The propagation of acoustic waves down the lines of force is analogous to the propagation of sound waves in an ear trumpet because of the convergence of the lines of force. As a result of this convergence, sound waves of finite amplitude should develop along the lines of force. The high viscosity in the outer exosphere controls the diffusion of the wave in this region. The breakup of the finite-amplitude viscosity-controlled wave as it enters the ionosphere may be important in auroral phenomena. 


\section{PART I}

\section{SLOWNESS AND PHASE SURFACES}

The propagation of magnetohydrodynamic waves is anisotropic. In addition, even in the absence of dissipation, waves are dispersed at low frequencies because of the action of gravity. The description of propagation in an anisotropic medium is aided by the construction of slowness surfaces in wave-number space at a fixed frequency and the inversion of these surfaces to form surfaces of constant phase. In this section certain of the geometrical features of the slowness surface and the surface of constant phase are reviewed.

The propagation of a wave can be described in terms of a phase function $\phi\left(x_{i}, t\right)$. The surface

$$
\phi\left(\mathbf{x}_{i}, t\right)=\text { constant }
$$

at a given time might correspond to a wave crest or trough. The phase velocity $C_{i}$ is the velocity of the surface; the wave number $k_{i}$ is defined as the normal to the phase surface,

$$
k_{i}=\frac{\partial \phi}{\partial x_{i}}
$$

and is in the direction of the phase velocity. The magnitude of the phase velocity is

$$
C_{i}=-\frac{\frac{\partial \phi}{\partial t}}{\frac{\partial \phi}{\partial x_{i}}}=\frac{\omega}{k_{i}} \text {, }
$$

where the frequency $\omega$ is defined by $\omega=-\partial \phi / \partial t$. The wave crests and troughs are at right angles to the vector $k_{i}$ and the wavelength is $2 \pi /\left|k_{i}\right| \cdot$

The equations for a given problem define a dispersion relation, a relation between $\mathrm{k}_{\mathbf{i}}, \mathbf{x}_{\mathbf{i}}$, and $\omega$. For convenience, the dispersion relation is written in the form

$$
W\left(\omega, k_{i}, x_{i}\right)=0 .
$$


The group velocity $u_{i}=d x_{i} / d t$ is determined from

$$
\mathbf{U}_{i}=-\frac{\frac{\partial W}{\partial k_{i}}}{\frac{\partial W}{\partial \omega}} .
$$

The existence of a scalar function $\phi$ corresponds to the conservation of the number of waves, or equivalent of $k_{i}$. The existence of $\phi$ implies

$$
\frac{\partial^{2} \phi}{\partial x_{i} \partial t}-\frac{\partial^{2} \phi}{\partial t \partial x_{i}}=0
$$

or

$$
\frac{\partial k_{i}}{\partial t}+\frac{\partial \omega}{\partial x_{i}}=0 .
$$

When Equations 2 and 3 are combined, the equation for the conservation of wave number takes the form

$$
-\frac{\partial k_{i}}{\partial t}+U_{j} \frac{\partial k_{i}}{\partial x_{j}}=-\frac{\frac{\partial W}{\partial x_{i}}}{\frac{\partial W}{\partial \omega}} .
$$

in which use has been made of the fact that $k_{i}$ is an irrotational vector (Equation 1). Equation 4 can be regarded as a form of Hamilton's equation (References 22 and 23). The left-hand side is the rate of change of the wave number following a point moving with velocity $U_{j}$, the velocity of a group of waves having the wave number $k_{1}$. The number of waves is conserved, provided the dispersion relation is independent of the spatial coordinate; the right-hand side of Equation 4 vanishes. The coefficients of the partial differential equation describing the general physical situation will be a function of position, and the right-hand side will be finite. The wave number and frequency must then be interpreted as the local wave propagation vector and frequency. These quantities can be determined by local arguments, and their propagation in space is then fixed by Equation 4. Once $k_{i}$ is determined in space by the dispersion relations and Equation 4 , it is possible to determine the phase function from Equation 1.

The foregoing approach yields a rigorous description of wave propagation, provided that a phase function exists, while the usual treatment of wave propagation in an inhomogeneous medium involves two kinds of approximations. 
The field variable can be written as the product of an amplitude function $\mathrm{F}$ and a phase function $\mathrm{G}$ :

$$
V=F\left(x_{j}, t\right) G\left[\phi\left(x_{i}, t\right)\right] \text {. }
$$

The variation of the field in time and space is

$$
\begin{aligned}
& \frac{\partial V}{\partial t}=\frac{\partial F}{\partial t} G-F \frac{\partial G}{\partial \phi} \omega, \\
& \frac{\partial \mathbf{V}}{\partial \mathbf{x}_{i}}=\frac{\partial F}{\partial \mathbf{x}_{i}} G+k_{i} F \frac{\partial G}{\partial \phi} .
\end{aligned}
$$

The J.W.K.B. approximation neglects the first term in comparison to the second term on the right-hand side of these two equations. This implies that the typical length scale associated with the spatial inhomogeneity is large compared to the wavelength. For an infinite medium the phase function $G$ can be taken as $G=\exp (i \phi)$ with the result that the operations of differentiation with respect to time and space yield

$$
\begin{aligned}
& \frac{\partial \mathrm{V}}{\partial \mathbf{t}}=-\mathbf{i} \omega \mathrm{V}, \\
& \frac{\partial \mathrm{V}}{\partial \mathbf{x}_{\mathbf{i}}}=i \mathrm{k}_{\mathbf{i}} \mathrm{V} .
\end{aligned}
$$

Under a similar but not identical approximation, the phase function is assumed linear in $\mathbf{k}_{\mathbf{i}}$ and $\omega$. Let $\mathrm{L}$ be the length scale in a given direction. Then for times limited by Ut $\ll L$ the phase function is approximately $\phi=k_{i} x_{i}-\omega t$.

In the problems treated next, the dispersion relation will be a function of a single coordinate, say $x_{3}$, and then the phase function will be of the form

$$
\phi=\mathrm{k}_{1} \mathrm{x}_{1}+\mathrm{k}_{2} \mathrm{x}_{2}+\int \mathrm{k}_{3} \mathrm{ax}_{3}-\omega t .
$$

At a given frequency and point in space it is possible to construct a three-dimensional surface in wave number space, determined by

$$
W\left(\omega, k_{i}, x_{i}\right)=0 .
$$

This surface will be termed the slowness surface (Reference 24). Every point on the slowness surface corresponds to a wave. Some of these waves, however, are disallowed 
because of the radiation condition, which allows only the propagation of energy outwards from the source in an initial-value problem.

The surface of constant phase, which can be constructed from the slowness surface, is the envelope at a given time of waves originating from a source at time $t=0$. The direction of the wave resulting from a point on the slowness surface is along the normal to the slowness surface. It should be noted that the direction of the wave number $k_{i}$ and the normal to the slowness surface need not be the same. The crests and troughs of the waves are then not necessarily at right angles to the direction of propagation. Since the normal to the slowness surface is parallel to the direction $x_{i}$ we have

$$
\mathbf{x}_{\mathbf{i}}=\operatorname{constant} \frac{\partial \mathrm{W}}{\partial \mathrm{k}_{\mathrm{i}}},
$$

where, at unit time,

$$
k_{i} x_{i}=\omega .
$$

Combining Equations 5 and 6 gives the surface of constant phase at unit time:

$$
\mathbf{x}_{i}=-\frac{\frac{\partial W}{\partial k_{i}}}{\frac{\partial W}{\partial \omega}} .
$$

At unit time the surface of constant phase is, then, a representation of the group velocity. This interpretation runs into difficulty, however, when the group velocity is a function of the spatial coordinate; in that case only the initial waveform can be calculated, and the waveform is distorted as the wave progresses through the medium. A further complication arises from dispersion. Waves are dispersed if $w\left(k_{i}, x_{j}, \omega\right)$ is not homogeneous in $\mathrm{k}_{\mathrm{i}}$ and $\omega$; and the phase surface for different frequencies will spread in time. A pulse contains a range of frequencies, and there will be a range of phase velocities for a fixed direction.

Once the slowness surface is determined, the surface of constant phase can be calculated by using Equation 7. A geometrical interpretation of Equation 7 is that the vector $\mathbf{x}_{i}$ is in the direction of a perpendicular dropped from the tangent plane of the slowness surface to the origin; and the magnitude of $x_{i}$ is proportional to the frequency and inversely proportional to the length of the perpendicular. The vector $x_{i}$ is the pole, with respect to a sphere of radius $\omega^{1 / 2}$ centered at the origin, of the tangent plane to the slowness surface; and this pole of the tangent plane is then the reciprocal pole of the slowness surface with respect to the sphere of radius $\omega^{1 / 2}$. The description of the wave 
number surface as a slowness surface results from the fact that the phase surface represents the progress of the wave front in time, and its reciprocal is the surface in wave number space.

The geometrical characteristics of the slowness surface determine the geometrical configuration of the surface of constant phase. A qualitative picture of the surface of constant phase can be obtained from the slowness surface without detailed calculations if certain geometrical correspondences are noted. Each tangent plane to the slowness surface corresponds to a point on the surface of constant phase. Reciprocally, to every tangent plane on the surface of constant phase there corresponds a point on the slowness surface. A tangent plane at a point $P$ of the slowness surface with $\mathrm{N}$ contacts corresponds to a point on the wave surface with $\mathrm{N}$ tangent planes. A parabolic point - i.e., a point at which one or both of the principal curvatures vanish (References 25 and 26) - on the slowness surface is reciprocal to a point on an edge of regression on the surface of constant phase. Further, if a tangent plane on the slowness surface touches the surface on a curve of tangency, all points on the curve correspond to a single point (conical point) on the constant phase surface. As an illustration of these geometrical correspondences, consider a plane with a circular bump in it. In the region away from the bump, the tangent plane to the slowness surface is in contact at innumerable points. This tangent plane corresponds to a single point on the surface of constant phase. Associated with the bump will be a circle of parabolic points which maps into a circular cusp in the constant phase surface. As the bump is reduced and approaches a plane, the surface of constant phase approaches a point. If the slowness surface is a plane, then the propagation is one-dimensional.

A picture of the relative amplitudes of waves traveling in different directions can also be obtained from a consideration of the slowness surface. Each tangent plane to the slowness surface corresponds to a wave, and a tangent plane with many points of contact represents the sum of these waves. The amplitude of that part of the phase surface corresponding to the tangent plane with many points of contact should exceed the amplitude of that part of the phase surface resulting from a tangent plane with a single point of contact to the slowness surface. As an example, the conical point on the surface of constant phase corresponding to a curve of tangency on the slowness surface should have a larger amplitude after a given distance of propagation than have the corresponding points on the constant phase surface with only a single tangent point. If the slowness surface is a sphere, then the corresponding surface of constant phase is also a sphere, and the amplitude decays as $1 / \mathrm{r}$. If, on the other hand, the surface is a plane, there is no geometrical attenuation. These two cases provide limits for the geometrical attenuation of waves. Lighthill (Reference 27), in a detailed investigation using the saddle point method in the asymptotic evaluations, finds that points on the cuspidal edge decay as $r^{-5 / 6}$ while conical points decay as $\mathbf{r}^{-1 / 2}$. 
The determination of the surface of constant phase can be similarly made for dispersive waves, but the spread in time must be considered. If the surface of constant phase is a sphere, the disturbance grows as a hollow spherical shell and the geometric attenuation is $\mathrm{r}^{-3 / 2}$.

The geometrical correspondences between the slowness surface and the surface of constant phase will be illustrated in detail in the treatment of hydromagnetic waves perturbed by gravity. 


\section{PART II}

\section{PARALLEL MAGNETIC AND GRAVITATIONAL FIELDS}

\section{Equations of Motion}

The simplest description of the motion of an electrically conducting fluid in the presence of a gravitational field with a potential $g z$ is

$$
\begin{aligned}
& \frac{d v_{i}}{d t}=-g \frac{\partial z}{\partial \mathbf{x}_{i}}-\frac{1}{\rho} \frac{\partial p}{\partial \mathbf{x}_{i}}-\frac{1}{4 \pi \mu \rho}\left(B_{j} \frac{\partial B_{j}}{\partial x_{i}}-B_{j} \frac{\partial B_{i}}{\partial x_{j}}\right), \\
& \frac{d B_{i}}{d t}=B_{j} \frac{v_{i}}{\partial x_{j}}-B_{i} \frac{\partial v_{j}}{\partial x_{j}}, \\
& \frac{d \rho}{d t}+\rho \frac{\partial v_{i}}{\partial \mathbf{x}_{i}}=0, \\
& \frac{d s}{d t}=-\frac{q}{T},
\end{aligned}
$$

where $\mathrm{g}$ is the gravitational constant at some reference altitude and $\mathrm{z}\left(\mathrm{x}_{\mathbf{i}}\right)$ is the gravitational altitude. A source term $q$, the net acession of heat, is included in the equation of entropy conservation (Equation 11).

The description yields a good approximation, provided a number of restrictions are met:

1. There exists a scalar fluid pressure $p$ which is a function of the entropy $s$ and density $\rho$.

2. Ohm's law is taken in the form $B_{i}=\epsilon_{i j k} B_{j} v_{k}$.

3. The usual assumptions of magnetohydrodynamics are made in that the displacement current is neglected and all velocities are small compared with the velocity of light.

4. Dissipation due to the finite viscosity and thermal conductivity of the fluid is neglected. In addition, Coriolis forces are neglected, though 
the effects of rotation on the gravitational field can be included by appropriate choice of the gravitational altitude.

The equation of the conservation of mass (Equation 10) can be combined with the thermodynamic relations to relate pressure and density variations. Thus,

$$
\frac{\mathrm{dp}}{\mathrm{dt}}-\mathrm{C}^{2} \frac{\mathrm{d} \rho}{\mathrm{dt}}=\frac{\rho(\gamma-1)}{a} \frac{\mathrm{q}}{\mathrm{T}}
$$

where $\mathrm{C}$ is the velocity of sound while $y=c_{p} / c_{v}$ is the ratio of the specific heats.

The set of non-linear equations (Equations 8 through 12) can be reduced to a tractable linear form by the perturbation scheme

$$
\begin{array}{ll}
\rho=\rho_{0}+\rho_{1}+\cdots & \mathrm{q}=\mathrm{q}_{1}+\cdots \\
\mathbf{s}=\mathrm{s}_{0}+\mathrm{s}_{1}+\cdots & \mathrm{B}_{\mathrm{i}}=\mathrm{B}_{\mathrm{i}}^{0}+\mathrm{B}_{\mathrm{i}}^{-1}+\cdots \\
\mathrm{p}=\mathrm{p}_{0}+\mathrm{p}_{1}+\cdots & \mathrm{v}_{\mathrm{i}}=\mathrm{v}_{\mathrm{i}}^{0}+\cdots
\end{array}
$$

where the quantities $\mathrm{p}_{0}, \mathrm{~B}_{\dot{i}}^{0}$, etc., represent the initial state of the fluid and field. Maxwell's equations (Equation 9) are satisfied identically to zero order.

Equation 8 yields

$$
\frac{\partial \mathrm{p}_{0}}{\partial \mathbf{x}_{\mathrm{i}}}=-\rho_{0} \mathrm{~g}\left(\frac{\partial \mathbf{z}}{\partial \mathbf{x}_{i}}\right)-\frac{1}{4 \pi \mu}\left(\frac{1}{2} \frac{\partial\left(\mathrm{B}_{\mathrm{j}}^{0}\right)^{2}}{\partial \mathbf{x}_{\mathrm{i}}}-\mathrm{B}_{\mathrm{j}}^{0} \frac{\partial \mathrm{B}_{\mathrm{i}}^{0}}{\partial \mathbf{x}_{\mathrm{j}}}\right)
$$

The relative magnitude of the two terms on the right-hand side of Equation 13 determines whether the initial particle distribution is gravitationally or magnetically controlled. For the present purposes the usual assumption that gravity determines the particle distribution over most of the exosphere is made. This need not hold true, and the particle distribution may be influenced greatly by the magnetic field.

The equations, to the first order, are then

$$
\begin{aligned}
& \frac{\partial B_{1}}{\partial t}=B_{j}^{0} \frac{\partial v_{i}}{\partial x_{j}}+B_{i}^{0} \frac{\partial v_{j}}{\partial x_{i}}-v_{j} \frac{\partial B_{i}^{0}}{\partial x_{j}}
\end{aligned}
$$

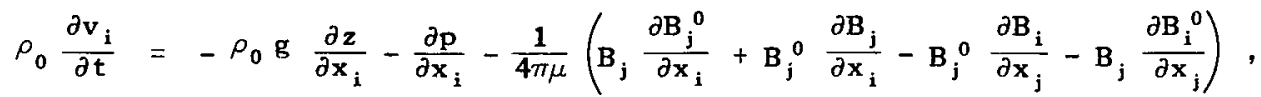


14

$$
\begin{aligned}
& \frac{\partial p}{\partial t}+v_{i} \frac{\partial p_{0}}{\partial x_{i}}=-\rho_{0} C_{0}^{2} \frac{\partial v_{i}}{\partial x_{i}}, \\
& \frac{\partial s}{\partial t}+\frac{\partial s_{0}}{\partial x_{i}} v_{i}=\frac{q_{i}}{T_{0}},
\end{aligned}
$$

where the unprimed variables represent the perturbed quantities. The initial pressure and entropy gradients, which appear in these first order equations, are not independent. Using the standard thermodynamic relations

$$
\begin{aligned}
& \frac{\partial \mathrm{T}_{0}}{\partial \mathbf{x}_{\mathrm{i}}}=\frac{\left(\gamma_{0}-1\right)}{a_{0}} \frac{\partial \rho_{0}}{\partial \mathbf{x}_{\mathrm{i}}}+\frac{\mathrm{T}_{0}}{\mathbf{c}_{\mathrm{v}}{ }^{0}} \frac{\partial \mathrm{s}_{0}}{\partial \mathbf{x}_{1}}, \\
& \frac{\partial \mathrm{p}_{0}}{\partial \mathbf{x}_{\mathrm{i}}}=\mathrm{C}_{0}{ }^{2} \frac{\partial \rho_{0}}{\partial \mathbf{x}_{\mathrm{i}}}+\frac{\left(\gamma_{0}-1\right)}{a_{0}} \rho_{0} \frac{\partial \mathbf{s}_{0}}{\partial \mathbf{x}_{\mathbf{i}}},
\end{aligned}
$$

we obtain for the entropy gradient

$$
\frac{\partial \mathrm{s}_{0}}{\partial \mathrm{x}_{i}}=\frac{\mathrm{c}_{\mathrm{p}}{ }^{0}}{\mathrm{~T}_{0}}\left(\frac{\partial \mathrm{T}_{0}}{\partial \mathrm{x}_{\mathrm{i}}}+\frac{\mathrm{g}_{0}{ }^{a} \mathrm{~T}_{0}}{\mathrm{c}_{\mathrm{p}}{ }^{0}} \frac{\partial z}{\partial \mathrm{x}_{\mathrm{i}}}\right),
$$

where the adiabatic temperature gradient is

$$
\frac{\partial T_{A}}{\partial x_{i}}=\frac{g a_{0} T_{0}}{c_{p}^{0}} \frac{\partial z}{\partial x_{i}},
$$

and $a_{0}$ is the coefficient of thermal expansion in the initial state. The velocity of sound, coefficient of thermal expansion, and heat capacity at constant pressure satisfy the simple relations

$$
\mathrm{C}_{0}^{2}=\gamma_{0} \mathrm{RT}_{0}, \quad \mathrm{c}_{\mathrm{v}}{ }^{0}=\frac{\mathrm{R}}{\left(\gamma_{0}-1\right)}, \quad a_{0}=\mathrm{T}_{0}^{-1},
$$

in an ideal gas. An ionized gas composed equally of electrons and singly ionized atoms has an effective value $\gamma_{0}=4 / 3$, since the value of $\gamma_{0}$ for the ions is $5 / 3$ and the electrons will behave more or less isothermally corresponding to $\gamma_{0}=1$. 


\section{Combined Magnetic and Gravitational Fields}

A marked simplification occurs in the case of a constant magnetic field with lines of force parallel to the gravitational field: Since the fields and motions are cylindrically symmetrical, the initial magnetic field is taken along the $x_{3}$ axis and the effects of curvature of the equipotential surfaces are neglected. Thus,

$$
\begin{aligned}
\mathbf{B}_{\mathbf{i}}{ }^{0} & =\left(0,0, \mathbf{B}^{0}\right), \\
\frac{\partial \mathbf{z}}{\partial \mathbf{x}_{\mathbf{i}}} & =\left(0,0, \frac{\partial \mathbf{z}}{\partial \mathbf{x}_{3}}\right) .
\end{aligned}
$$

The first order perturbation equations reduce to

$$
\begin{aligned}
& \frac{\partial \mathrm{b}_{1}}{\partial t}=\frac{\partial \mathrm{v}_{1}}{\partial \mathrm{x}_{3}}, \quad \frac{\partial \mathrm{b}_{2}}{\partial \mathrm{t}}=\frac{\partial \mathrm{v}_{2}}{\partial \mathrm{x}_{3}}, \quad \frac{\partial \mathrm{b}_{3}}{\partial \mathrm{t}}=-\left(\frac{\partial \mathrm{v}_{1}}{\partial \mathrm{x}_{1}}+\frac{\partial \mathrm{v}_{2}}{\partial \mathrm{x}_{2}}\right) \text {, } \\
& \frac{\partial v_{1}}{\partial \mathrm{t}}+\frac{1}{\rho_{0}} \cdot \frac{\partial \mathrm{p}}{\partial \mathrm{x}_{\mathrm{i}}}+\mathrm{C}_{\mathrm{A}}^{2}\left(\frac{\partial \mathrm{b}_{3}}{\partial \mathrm{x}_{1}}-\frac{\partial \mathrm{b}_{1}}{\partial \mathrm{x}_{3}}\right)=0 \\
& \frac{\partial v_{2}}{\partial t}+\frac{1}{\rho_{0}} \frac{\partial \mathrm{p}}{\partial \mathrm{x}_{2}}+\mathrm{C}_{\mathrm{A}}^{2}\left(\frac{\partial \mathrm{b}_{3}}{\partial \mathrm{x}_{2}}-\frac{\partial \mathrm{b}_{2}}{\partial \mathrm{x}_{3}}\right)=0 \\
& \frac{\partial \mathrm{v}_{3}}{\partial \mathrm{t}}+\frac{1}{\rho_{0}} \frac{\partial \mathrm{p}}{\partial \mathrm{x}_{3}}+\frac{1}{\rho_{0}} \frac{\mathrm{g}}{\mathrm{C}_{0}^{2}} \mathrm{p}-\mathrm{N}^{2} \mathrm{\rho}=0 \\
& \frac{\partial \mathrm{p}_{3}}{\partial t}+\mathrm{g} \rho_{0} \mathrm{v}_{3}+\rho_{0} \mathrm{C}_{0}^{2}\left(\frac{\partial \mathrm{v}_{1}}{\partial \mathrm{x}_{1}}+\frac{\partial \mathrm{v}_{2}}{\partial \mathrm{x}_{2}}+\frac{\partial \mathrm{v}_{3}}{\partial \mathrm{x}_{3}}\right)=0 \\
& \frac{\partial Q}{\partial \mathrm{t}}+\mathbf{v}_{3}=0
\end{aligned}
$$

where

$$
b_{i}=\frac{B_{i}}{\left|B_{j}{ }^{0}\right|}
$$

is the normalized fluctuation in the magnetic field; 


$$
\mathbf{C}_{\mathbf{A}}=\frac{\left|\mathbf{B}_{\mathbf{i}}^{0}\right|}{\left(4 \pi \mu \rho_{0}\right)^{\frac{1}{2}}}
$$

is the Alfvén wave velocity in the undisturbed medium;

$$
Q=S \frac{\frac{\partial z}{\partial x_{i}}}{\frac{d s_{0}}{d x_{i}}}
$$

is the normalized fluctuation in entropy; and $N$ is the Väisälä frequency (Reference 21) given by

$$
\mathbf{N}^{2}=g \frac{\partial \mathbf{s}_{0}}{\partial \mathbf{x}_{i}} \frac{\gamma_{0}-1}{a_{0} c_{0}{ }^{2} \frac{\partial z}{\partial \mathbf{x}_{i}}}
$$

The Väisälä frequency $N$ is interpreted here as the frequency of oscillation of a small mass of fluid when displaced from its zero-order position in the gravitational field and allowed to move freely. As will be shown, motions with frequencies that are large compared to $\mathrm{N}$ are not influenced by gravity. In a perfect gas the Väisälä frequency takes the form

$$
N^{2}=g \frac{\frac{\mathrm{dT}_{0}}{\mathrm{~d} z}+\frac{\mathrm{g}(\gamma-1)}{\gamma \mathrm{R}}}{\mathrm{T}_{0}}
$$

and, if the fluid is isothermal, then

$$
\mathrm{N}^{2}=\frac{\mathrm{g}^{2}}{\mathrm{C}_{0}^{2}}\left(\gamma_{0}-1\right)
$$

Equations 14, 15, 16, and 17 form a coupled set for $\mathrm{v}_{i}$. Their complexity is reduced, for a homogeneous fluid, by choosing a new set of variables; and more importantly, one mode of motion is separable (References 20, 28, and 29). A similar separation is possible in the presence of a gravitational field, provided that gravity and the lines of force are parallel. Consider a motion in which $p=0, v_{3}=0, b_{3}=0$. The pressure equation then implies

$$
\frac{\partial v_{1}}{\partial x_{2}}+\frac{\partial v_{2}}{\partial x_{2}}=0
$$


and there is no variation of entropy, i.e.,

$$
\frac{\partial Q}{\partial t}=0 ; \quad 0=0
$$

Equations 14 and 15 combine to give

$$
\frac{\partial \xi}{\partial t}=C_{A}^{2} \frac{\partial_{j}}{\partial x_{3}}, \quad \frac{\partial j}{\partial t}=\frac{\partial \xi}{\partial x_{3}}
$$

where $\xi$ is the vorticity, or spin, of the field about the line of force and $j$ is the normalized current density:

$$
\xi=\frac{\partial \mathrm{v}_{2}}{\partial \mathrm{x}_{1}}-\frac{\partial \mathrm{v}_{1}}{\partial \mathrm{x}_{2}}, \quad \quad \mathrm{j}=\frac{\partial \mathrm{b}_{2}}{\partial \mathrm{x}_{1}}-\frac{\partial \mathrm{b}_{1}}{\partial \mathrm{x}_{2}} .
$$

Equations 18 yield

$$
\frac{\partial^{2} \xi}{\partial t^{2}}=C_{A}^{2} \frac{\partial^{2} \xi}{\partial x_{3}^{2}}, \quad \frac{\partial^{2} j}{\partial t^{2}}=\frac{\partial}{\partial x_{3}}\left(C_{A}^{2} \frac{\partial j}{\partial x_{3}}\right)
$$

The vorticity about the line of force is propagated along the line of force at the Alfvén wave velocity as a one-dimensional wave. The thermodynamic state of the system remains constant, since the variations in entropy and pressure vanish. The wave velocity varies with the density of the fluid.

The $\mathbf{P}$ and $\mathbf{T}$ modes are not separable. The variation of pressure, longitudinal component of the magnetic field, component of velocity along the magnetic field, and entropy in these modes are governed by the system of equations

$$
\begin{aligned}
\left.\frac{\partial^{2} \mathrm{~b}_{3}}{\partial \mathrm{t}^{2}}-\frac{1}{\rho_{0}}\left(\frac{\partial^{2} \mathrm{p}}{\partial \mathrm{x}_{1}{ }^{2}}+\frac{\partial^{2} \mathrm{p}}{\partial \mathrm{x}_{2}{ }^{2}}\right)-\frac{\partial^{2} \mathrm{~b}_{3}}{\partial \mathrm{x}_{3}^{2}}\right) & =0, \\
\frac{\partial \mathrm{v}_{3}}{\partial \mathrm{t}}+\frac{1}{\rho_{0}}\left(\frac{\partial}{\partial \mathrm{x}_{3}}+\frac{\mathrm{g}}{\mathrm{C}_{0}{ }^{2}}\right) \mathrm{p}-\mathrm{N}^{2} \mathrm{Q} & =0, \\
\frac{\partial \mathrm{p}}{\partial \mathrm{t}}+\rho_{0} \mathrm{C}_{0}^{2}\left(\frac{\partial}{\partial \mathrm{x}_{3}}-\frac{\mathrm{g}}{\mathrm{C}_{0}{ }^{2}}\right) \mathrm{v}_{3}-\rho_{0} \mathrm{C}_{0}^{2} \frac{\partial \mathrm{b}_{3}}{\partial \mathrm{t}} & =0, \\
\frac{\partial \mathrm{Q}}{\partial \mathrm{t}}+\mathrm{v}_{3} & =0 .
\end{aligned}
$$

We note that Equation 19 reduces to a spherically spreading Alfvén wave if $p=0, g=0$. 
As a preliminary to the consideration of wave propagation in an inhomogeneous medium, consider Equation 19 for $\mathrm{g}=0$. The dispersion relation is

$$
k^{2}=\frac{\left(\omega^{2}-\mathrm{C}_{0}{ }^{2} \mathrm{k}_{3}^{2}\right)\left(\omega^{2}-\mathrm{C}_{\mathrm{A}}{ }^{2} \mathrm{k}_{3}{ }^{2}\right)}{\left(\mathrm{C}_{\mathrm{A}}{ }^{2}+\mathrm{C}_{0}{ }^{2}\right)^{2} \omega^{2}-\mathrm{C}_{\mathrm{A}}{ }^{2} \mathrm{C}_{0}{ }^{2} \mathrm{k}_{3}{ }^{2}},
$$

where

$$
\kappa^{2}=\mathrm{k}_{1}^{2}+\mathrm{k}_{2}^{2}
$$

The propagation is thus symmetrical about the $\mathrm{x}_{3}$ axis.

Given values of the wave number $k_{3}$ and frequency $\omega$, Equation 20 determines $k^{2}$. The value thus determined might be negative; but $\kappa$ must be real, since the system is conservative and is assumed stable. The values of frequency and wave number resulting in a negative $k^{2}$ do not give an allowed eigen solution. The regions in the $\omega-k_{3}$ plane of permitted waves are shown in Figure 1. The upper left-hand region of the diagram is

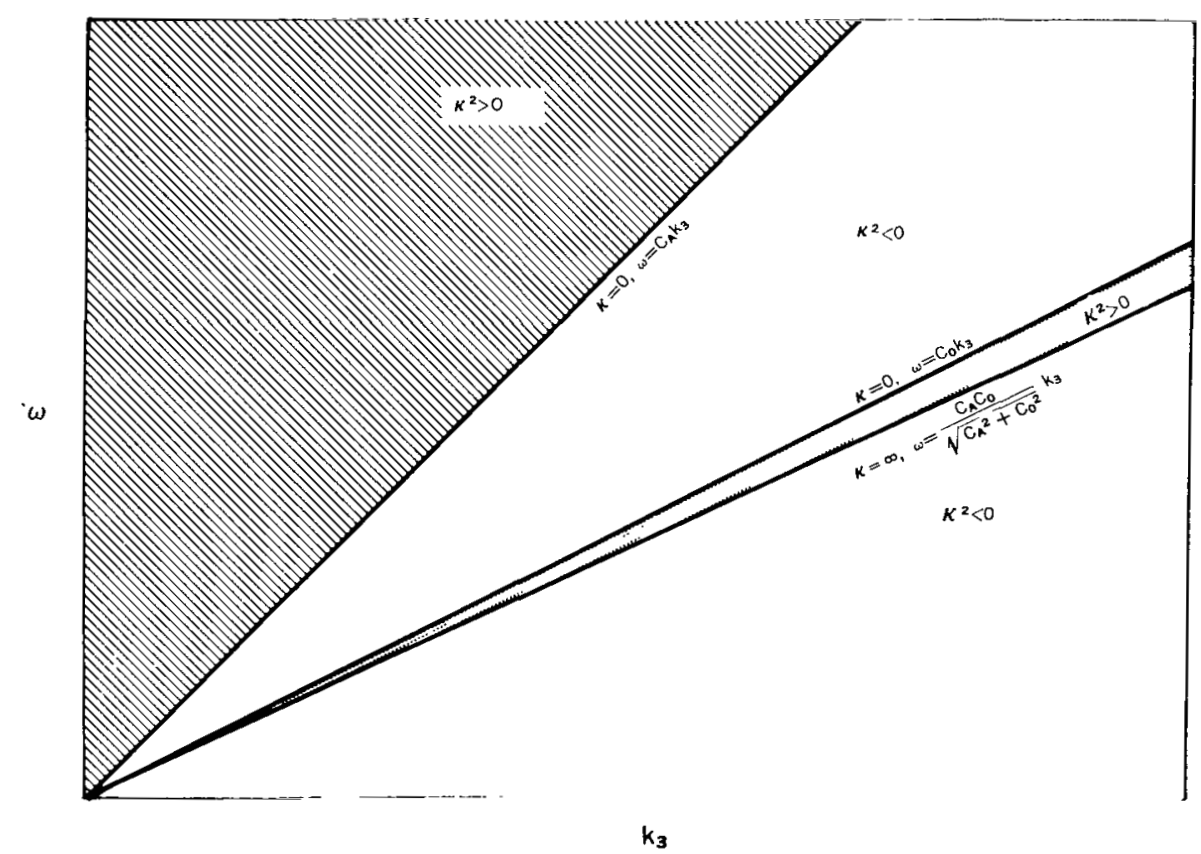

Figure 1 - Diagnostic diagram for hydromagnetic wave propagation in a uniform fluid. The diagram is scaled so that $C_{0} / C_{A}=0.5$. The upper region is a region of $T$ mode propagation. The narrow stippled region is the $P$ mode region. 
bounded by the line $\omega=\mathrm{C}_{\mathrm{A}} \mathrm{k}_{3}$. Over this region, $\kappa^{2}>0$. A second region of allowed solutions is bounded by the lines $\omega=C_{0} k_{3}$ and $\omega=k_{3} C_{A} C_{0} / \sqrt{C_{A}^{2}+C_{0}^{2}}$. Along this last line, the wave number $\kappa$ becomes infinite. This is interpreted physically as a vanishing wavelength.

The diagram is scaled so that $\beta=\mathrm{C}_{0} / \mathrm{C}_{\mathrm{A}}=0.5$. The upper left-hand region corresponds to the propagation of the $\mathrm{T}$ mode in which two-dimensional divergence is propagated at speeds comparable to the Alfvén wave velocity. The narrow region corresponds to the propagation of the $\mathrm{P}$ mode. The lines bounding the regions of allowed solutions are straight, since the dispersion relation is homogeneous in the frequency and wave number; the $\mathrm{T}$ and $\mathrm{P}$ modes are propagated in a unifor $\mathrm{m}$ medium without dispersion. It should be noted that the line $\omega=\mathrm{C}_{\mathrm{A}} \mathrm{k}_{3}$ corresponds to the $\mathrm{V}$ mode, which has been separated by the choice of variables.

The dispersion relation of Equation 20 may be written in the form

$$
\mathrm{W}\left(\mathrm{k}_{\mathrm{i}}, \omega\right)=\beta^{2} \mathrm{k}_{3}^{4}+\left[\beta^{2} \kappa^{2}-\left(\beta^{2}+1\right) l^{2}\right] \mathrm{k}_{3}{ }^{2}+l^{2}\left[l^{2}-\left(1+\beta^{2}\right) \kappa^{2}\right]=0,
$$

where $\omega=\mathrm{C}_{0} / \mathrm{C}_{\mathrm{A}}$, and $l=\omega / \mathrm{C}_{\mathrm{A}}$ so that the unit of distance is $\mathrm{C}_{\mathrm{A}} / \omega$.

The slowness surface corresponding to the diagnostic diagram of Figure 1 is shown for a fixed frequency $\omega$ in Figure 2. The complete surfaces are obtained by rotating the $\mathrm{k}_{3}-\kappa$ plane about the $\mathrm{k}_{3}$ axis and reflecting across the plane $\mathrm{k}_{3}=0$. The two sheets of the slowness surface determined by the dispersion relation of Equation 22 are a sphere flattened along the $k$ direction and a near-plane with a bump towards the origin. In addition, the plane representing the one-dimensional propagation of the vorticity ( $\mathrm{V}$ mode) has been added. The near-sphere corresponds to the spherically spreading $T$ mode. This is noted by allowing $\beta$ to approach zero in Equation 22. As $\beta$ approaches zero, the propagation surface becomes a sphere corresponding to the spherical propagation of Alfvén waves.

Substituting $\mathrm{k}_{3}=l$ into the pressure equation (Equation 19) we obtain

$$
\frac{\mathrm{p}}{\mathrm{P}_{\mathrm{m}}}=\frac{\beta^{2}}{1-\beta^{2}}
$$

where

$$
p_{m}=\rho_{0} C_{A}{ }^{2} b_{3}=\frac{B^{0} B_{3}{ }^{1}}{4 \pi \mu}
$$

is the variation in the magnetic pressure. 


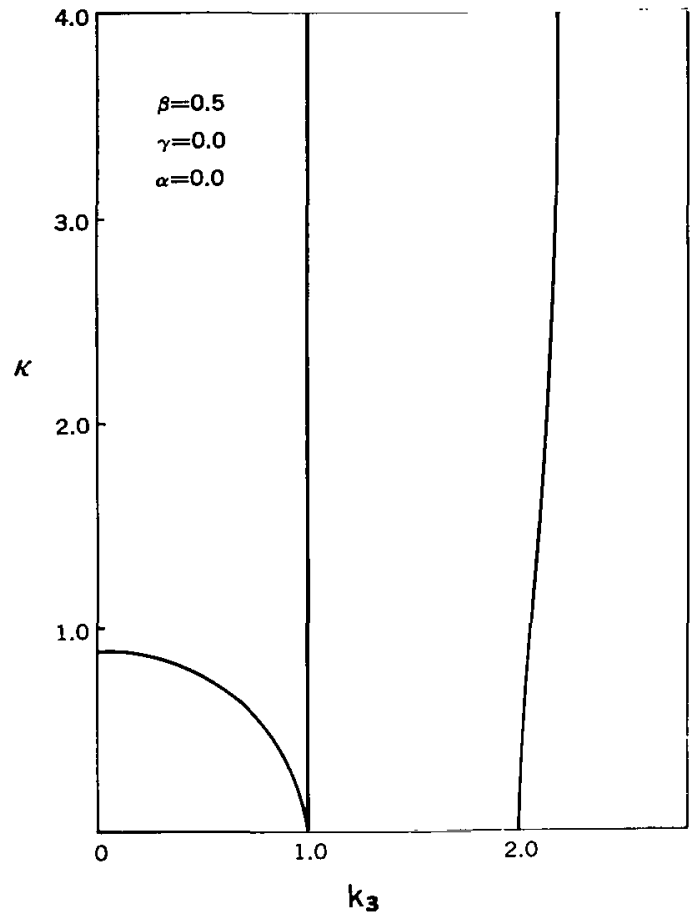

Figure 2 - Slowness surfaces for the propagation of hydromagnetic waves in a uniform medium. The complete surfaces are obtained by rotation about the $k_{3}$ axis and reflection across the $k$ plane. The unit of wave number is $\omega / C_{A}$. The lines of force are parallel to the $k_{3}$ direction. The near-sphere corresponds to a $T$ mode propagation; the plane at $k_{3}= \pm I$ is the $V$ mode propagation, and the plane with a bump describes the $P$ mode propagation.
Note that in the $\mathbf{T}$ mode a small fluctuation in particle pressure results in a larger fluctuation in the magnetic perturbation pressure, provided $\beta<1$. The $T$ mode propagates a fluctuation in the longitudinal magnetic field plus a slight condensation of the changed particles.

Over most of the planar sheet we have

$$
k_{3}=\omega \cdot\left(C_{0}^{-2}+C_{A}^{-2}\right)^{\frac{1}{2}},
$$

which corresponds to the one-dimensional propagation at a modified sound velocity

$$
\mathrm{C}_{\mathrm{phase}} \approx \frac{\omega}{\mathrm{k}_{3}}=\frac{\mathrm{C}_{\mathrm{A}} \mathrm{C}_{0}}{\left(\mathrm{C}_{0}{ }^{2}+\mathrm{C}_{\mathrm{A}}{ }^{2}\right)^{\frac{1}{2}}}
$$

In order to obtain the variation of pressure in the $\mathrm{P}$ mode we substitute Equation 23 into the pressure equation, with the result $\mathrm{p}+\mathrm{p}_{\mathrm{m}}=0$. Thus, the excess pressure $p$ and the magnetic pressure $p_{m}$ are in approximate balance in the $P$ mode. As $p$ drops and suction develops, a magnetic field is generated. As the particle pressure increases, energy is transferred from the magnetic to the acoustic field.

The $\mathrm{T}$ mode, with a nearly spherical slowness surface, will undergo geometrical attenuation as $1 / r$. The $V$ mode, with a planar slowness surface, undergoes no geometrical attenuation. The propagation of the $\mathbf{P}$ mode is more complicated.

\section{Spreading of the $\mathbf{P}$ Mode}

The surfaces of constant phase can be constructed from the slowness surfaces. Figure 3 shows the waveform at unit time for a source located at the origin at time $t=0$ and for $; 06,0.8$. The magnetic lines of force are taken along the $x_{3}$ axis, and there is cylindrical symmetry about the $x_{3}$ axis and across the $x_{2}-x_{3}$ plane. The unit of distance is ( ${ }_{A} t$. The slowness surfaces for the $V$ mode degenerate to a point on the $x_{3}$ axis. The $T$ mode, represented by a sphere flattened along the $\kappa$ direction in wave number

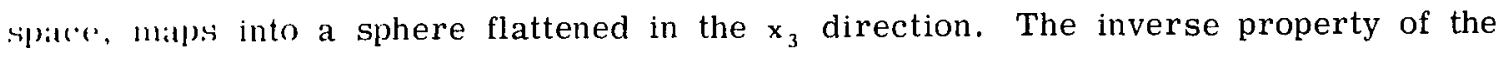




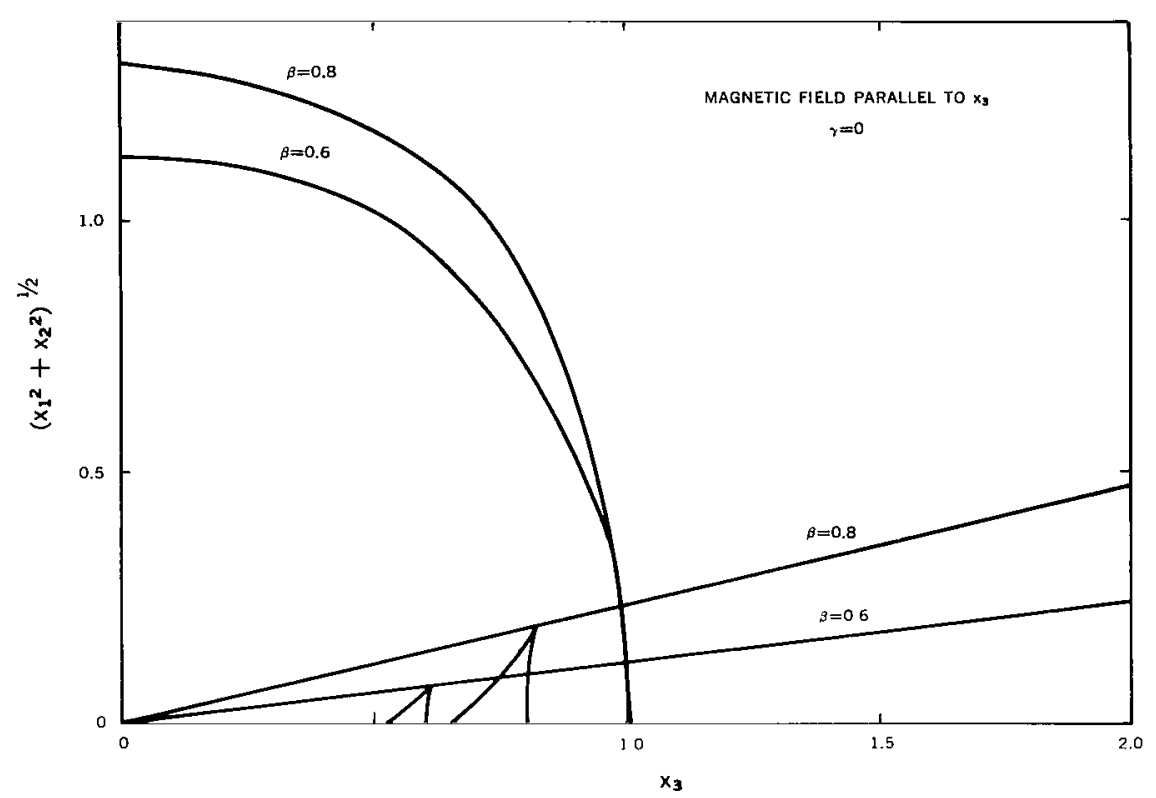

Figure 3 - Surfaces of constant phase for a uniform medium. The complete surfaces are obtained by rotation about the $x_{3}$ axis and reflection across the $x_{1}-x_{2}$ plane. The cusped triangles correspond to a $P$ wave, the flattened sphere to a $T$ wave.

slowness surface is illustrated in the wave surface for the $\mathrm{T}$ mode. The phase velocity in the direction perpendicular to the lines of force is greater than the velocity along the lines of force.

The one-dimensional $P$ mode has a constant phase surface in the form of a cusped surface with a conical point on the $\mathrm{x}_{3}$ axis. This cusped surface is subtended by a cone with a vertex at the origin. The angular diameter of the cone increases with increasing $\beta$ up to $\beta=1$.

For $\beta<1$ the propagation of the $\mathbf{P}$ mode is in some ways analogous to the phenomenon of conical refraction in optics made of anisotropic crystals (Reference 19). The slowness surface has a dimple which is concave in the direction of the magnetic field; the presence of this dimple leads to a circle of parabolic points, and it is these parabolic points that give rise to the cuspidal edge on the phase surface.

For large values of $\kappa$, the slowness surface becomes tangent to a plane perpendicular to the $x_{3}$ axis; this plane corresponds to the conical point on a surface of constant phase. The points along the cuspidal edge geometrically attenuate at a slower rate than 
do all other points on the surfaces excepting the conical point, which decays at the slowest rate. The $\mathrm{P}$ mode is then dominated by the cuspidal circle and conical point. A projection of the wave amplitude onto a plane perpendicular to the direction of propaga tion would show a circle around a central dot.

The transition from $\beta<1$ to $\beta>1$ is illustrated in Figure 4. As the sound velocity is increased at constant Alfvén wave velocity, the phase surface for the $\mathrm{P}$ mode becomes a flattened sphere and the phase surface for the $T$ mode is a cusped surface bounded by a cone with a vertex at the origin. The angular diameter of the cone bounding the $T$ mode becomes less with increasing $\beta$.

Over much of the exosphere, $\beta$ is very small. In the limit of small $\beta$ the cusped triangle generating the $\mathbf{P}$ phase surface reduces to a point maintaining the cuspidal form. Then the propagation of the $\mathrm{P}$ mode is very nearly one-dimensional. The $\mathrm{T}$ mode propagation becomes more nearly spherical as $\beta$ decreases. At high frequencies, energy may

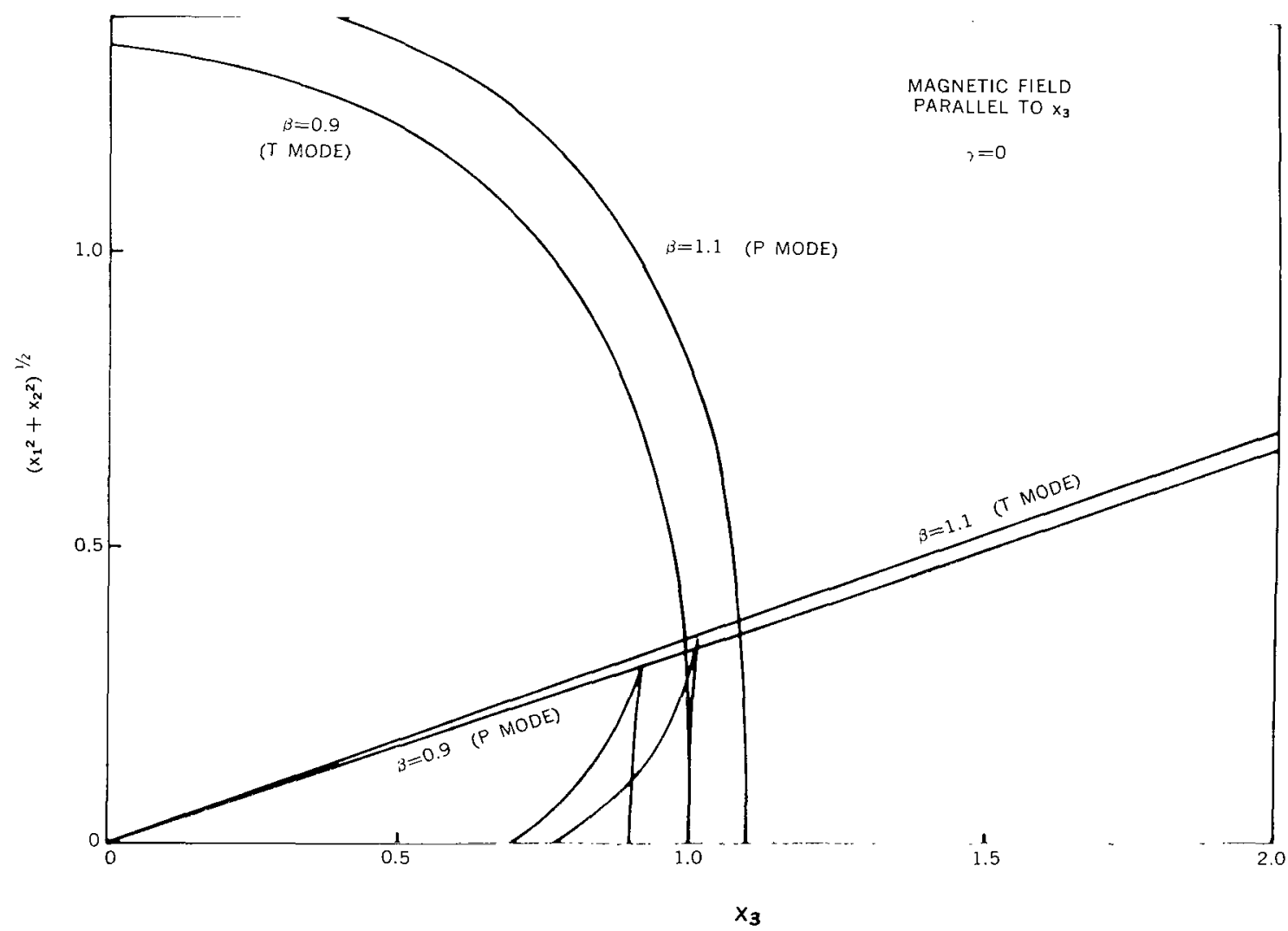

Figure 4 - Constant phase surfaces illustrating transistions from $T$ mode propagation to $P$ mode propagation 
be propagated in the exosphere by one-dimensional $\mathrm{V}$ waves, nearly one-dimensional $\mathrm{P}$ wave, and spherically spreading $\mathrm{T}$ wave.

\section{Gravitational Coupling}

The field equations (Equations 14 through 17) combine to yield the dispersion relation

$$
k^{2}=c \begin{gathered}
{\left[\left(\omega^{2}-\mathrm{N}_{1}{ }^{2}\right)-\mathrm{C}_{0}{ }^{2} \mathrm{k}_{3}{ }^{2}\right]\left[\omega^{2}-\mathrm{C}_{\mathrm{A}}{ }^{2} \mathrm{k}_{3}{ }^{2}\right]} \\
\left(\mathrm{C}_{0}{ }^{2}+\mathrm{C}_{\mathrm{A}}{ }^{2}\right) \omega^{2}-\mathrm{C}_{\mathrm{A}}{ }^{2} \mathrm{~N}_{1}{ }^{2}-\mathrm{C}_{0}{ }^{2} \mathrm{~N}^{2}-\mathrm{C}_{\mathrm{A}}{ }^{2} \mathrm{C}_{0}{ }^{2} \mathrm{k}_{3}{ }^{2}
\end{gathered},
$$

where $N_{1}$ is a modified Väisälä frequency and

$$
\mathrm{N}_{1}^{2}=\mathrm{N}^{2}+\frac{\mathrm{q}^{2}}{\mathrm{C}_{0}^{2}}
$$

It should be noted that the dispersion relation refers only to the coupled $\mathrm{P}$ and $\mathrm{T}$ modes. The $\mathrm{V}$ mode is unaffected by gravity, provided $\mathrm{g} \mathrm{d} z / \partial \mathrm{x}_{\mathrm{i}}$ is along the line of force.

The diagnostic diagram corresponding to Equation 24 is shown in Figure 5. The regions of allowed propagation are limited by the curves of vanishing wave number $k$,

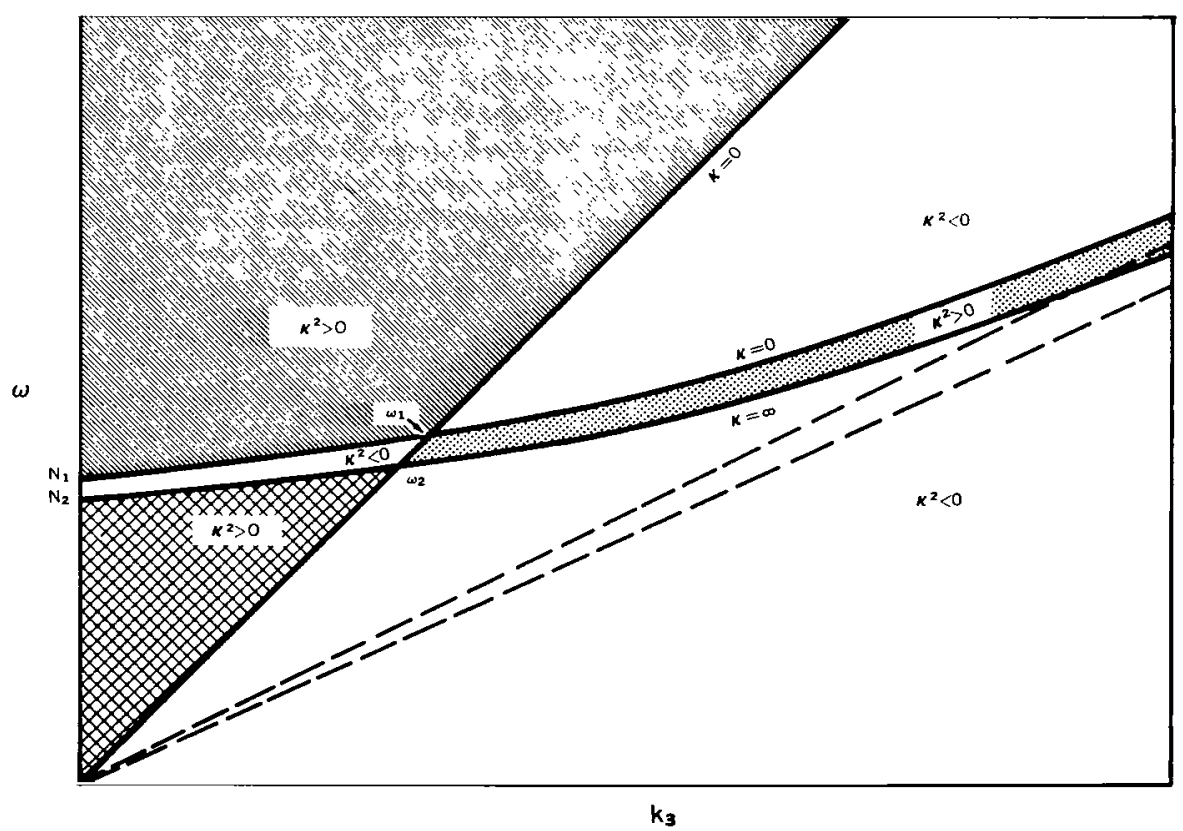

Figure 5 - Diagnostic diagram for hydromagnetic wave propagation with the lines of force parallel to the direction of gravity. The diagram is scaled with $\beta=0.5$. The upper left-hand region corresponds to perturbed $T$ mode propagation, the narrow stippled region to $P$ mode propagation, and the lower crosshatched region to gravitationally controlled propagation. 


$$
\begin{aligned}
\omega & =\mathrm{C}_{\mathrm{A}} \mathrm{k}_{3} \\
\omega^{2} & =\mathrm{N}_{1}{ }^{2}+\mathrm{C}_{0}{ }^{2} \mathrm{k}_{3}{ }^{2} ;
\end{aligned}
$$

and by the curve

$$
\left(\mathrm{C}_{\mathrm{A}}^{2}+\mathrm{C}_{0}^{2}\right) \omega^{2}-\mathrm{C}_{0}^{2} \mathrm{C}_{\mathrm{A}}^{2} \mathrm{k}_{3}^{2}-\mathrm{C}_{0}^{2} \mathrm{~N}^{2}-\mathrm{C}_{\mathrm{A}}^{2} \mathrm{~N}_{1}^{2}=0
$$

along which $\kappa$ becomes infinite, corresponding to zero wavelength. The curves bounding regions of allowed propagation are no longer straight lines, and the dispersion relation is not homogeneous in the wave number and frequency. For frequencies near $\mathrm{N}_{1}$, the propagation is dispersive.

At large frequencies and wave numbers $\kappa_{3}$, the diagnostic diagram is similar to that shown in Figure 1. There are two modes of propagation; a $\mathrm{P}$ mode and a $\mathrm{T}$ mode. Gravity dominates the propagation at low frequencies and large wave numbers. The characteristic frequencies noted in Figure 5 are $\mathrm{N}_{1}, \omega_{1}, \omega_{2}{ }^{2}$, and $\mathrm{N}_{2}{ }^{2}$, where

$$
\begin{gathered}
\omega_{1}=\frac{\mathrm{N}_{1}}{\left(1-\beta^{2}\right)^{\frac{1}{2}}}, \\
\omega_{2}^{2}=\mathrm{N}_{1}{ }^{2}+\beta^{2} \mathrm{~N}^{2}, \\
\mathrm{~N}_{2}{ }^{2}=\frac{\mathrm{C}_{0}{ }^{2} \mathrm{~N}^{2}+\mathrm{C}_{\mathrm{A}}{ }^{2} \mathrm{~N}_{1}{ }^{2}}{\mathrm{C}_{0}{ }^{2}+\mathrm{C}_{\mathrm{A}}{ }^{2}}=\frac{\mathrm{N}_{1}{ }^{2}+\beta^{2} \mathrm{~N}^{2}}{1+\beta^{2}} .
\end{gathered}
$$

For $\beta<1$, the relative magnitudes of the four frequencies are $N_{2}<N_{1}<\omega_{2}<\omega_{1}$.

The nature of the waves in the various regions of allowed propagation in the diagnostic diagram is best obtained by constructing the slowness surface and the corresponding surfaces of constant phase. The dispersion relation (Equation 24) is written in terms of the dimensional quantities $\gamma=\mathrm{N}_{1} / \omega$ and $a=\mathrm{N} / \omega$ as

$$
\beta^{2} \mathrm{k}_{3}^{4}-\left[l^{2}\left(1-\gamma^{2}\right)+\beta^{2}\left(l^{2}-\kappa^{2}\right)\right] \mathrm{k}_{3}^{2}+l^{2}\left(1-\gamma^{2}\right)\left(l^{2}-\kappa^{2}\right)-\beta^{2} l^{2}\left(1-\alpha^{2}\right) \kappa^{2}=0 .
$$

The wave numbers are thus measured in units of $l=\omega / \mathrm{C}_{\mathrm{A}}$.

The slowness surfaces for the values $\gamma=0.5$ and 0.9 are shown in Figure 6 . The value of $\beta$ is set at 0.5 . There is symmetry about the $k_{3}$ axis and across the $k_{1}-k_{2}$ 


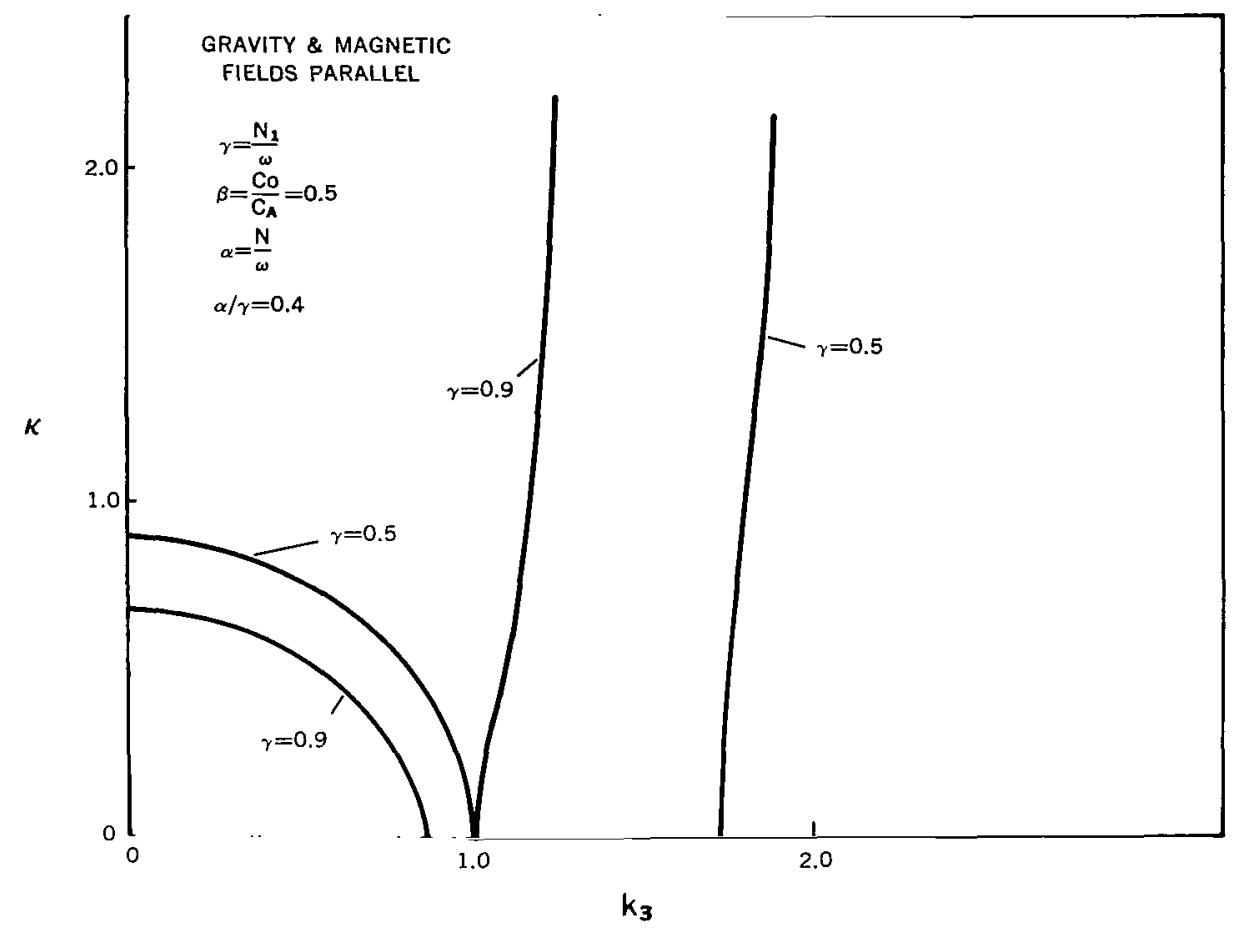

Figure 6 - Slowness surfaces for parallel gravity and magnetic fields. The complete surface is obtained by rotating about the $k_{3}$ direction and reflecting across the $k_{1}-k_{2}$ plane. The planar sheets correspond to perfurbed $P$ mode propagation, the distorted spheres to $T$ mode propagation. The propagation is dispersive.

plane. The propagation of both the modified $\mathrm{T}$ and $\mathrm{P}$ mode is dispersive, with the high frequency $\mathrm{T}$ and $\mathrm{P}$ components traveling at a slower rate than the low frequency components. In addition to the two modes shown, the $\mathrm{V}$ mode is propagated without dispersion. Comparing Figure 6 with Figure 2, we note that for $\gamma=0.5$ the $T$ mode is affected only slightly by gravity, while the $\mathrm{P}$ mode is markedly changed.

The dispersion resulting from gravity is clearly illustrated in Figure 7 where the constant phase surfaces corresponding to the slowness surfaces of Figure 6 are shown. The lower frequency $\gamma=0.9$ wave travels faster than the high frequency wave. In addition, the angular diameter of the cone enclosing the low frequency wave is larger. This corresponds to the fact that at low frequencies in the diagnostic diagram the lines bounding the region of propagation of the $\mathrm{P}$ wave become more nearly parallel to the $\mathrm{k}_{3}$ axis. The values of the critical frequencies are $\gamma_{1}=0.87, \gamma_{2}=0.954$, for $\beta=0.5$. At frequencies greater than $\gamma=0.87$, the $T$ mode is almost unperturbed by the action of gravity. The transition region between frequencies $\omega_{1}$ and $\omega_{2}$ is illustrated in Figure 8, where the slowness surfaces for $\gamma=0.9,0.95$, and 1.0 are shown. As the frequency decreases with increasing $\gamma$, the slowness surface for the $\mathbf{T}$ mode propagation shrinks to a point. At frequencies less than $\mathrm{N}_{1}$ there is no propagation in the $\mathrm{T}$ mode. At frequencies 


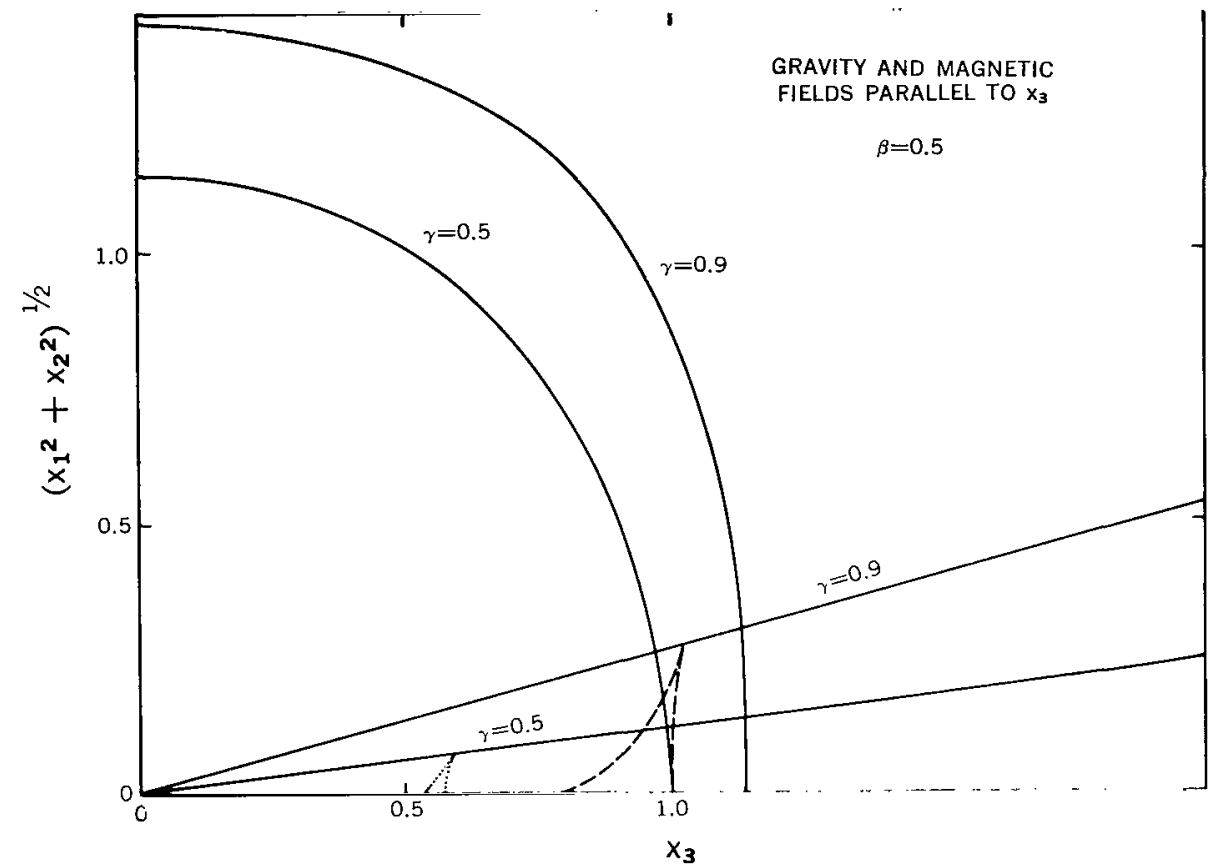

Figure 7-Constant phase surfaces corresponding to the slowness surfaces of Figure 6 . Both the $\mathrm{T}$ mode (nearly spherical phase surface) and the $\mathrm{V}$ mode (cusped surface) are highly dispersive.

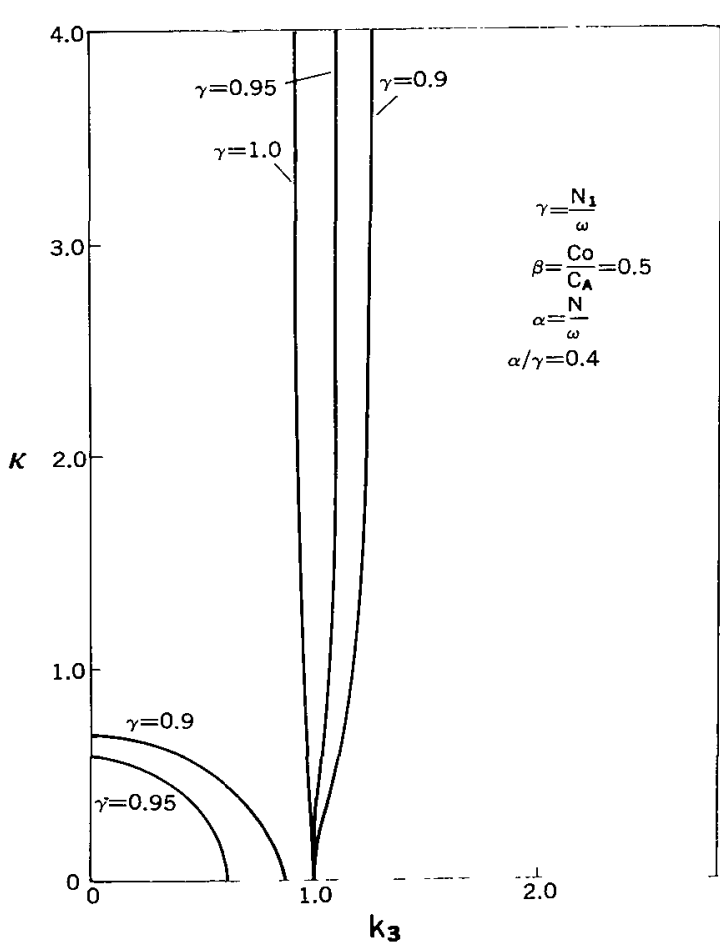

Figure 8 - Slowness surfaces at frequencies near to the critical frequency $N_{1}$. The complete surfaces are obtained by rotation about the $k_{3}$ axis and reflection across the $k_{1}-k_{2}$ plane.

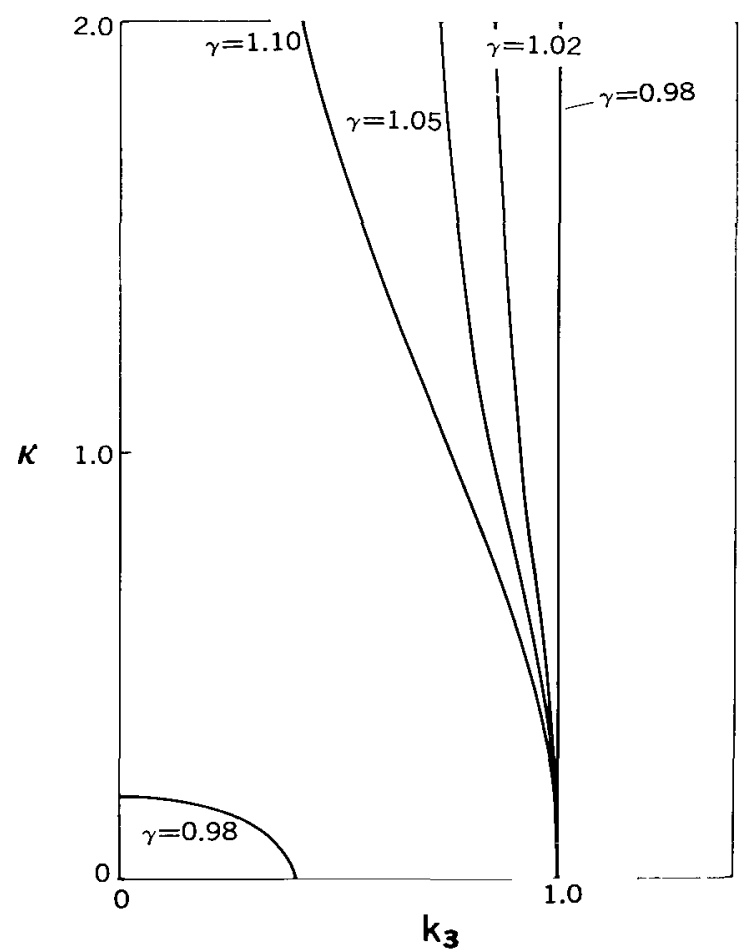

Figure 9 - Slowness surfaces at frequencies lower than $\mathrm{N}_{1}$. 
intermediate to $\omega_{2}$ and $N_{1}$, the propagation surface for the $T$ mode becomes greatly flattened in the $k$ direction (see also Figure 9).

There is no propagation of the $\mathrm{T}$ mode for frequencies less than $\omega_{2}$ where a new region appears in the diagnostic diagram. The slowness surfaces for the transition region $\omega_{2}-\mathrm{N}_{2}$ are shown in Figure 9 , and the corresponding surfaces of constant phase in Figure 10. As the frequency is lowered, the angular diameter of the cone bounding the surface of constant phase increases. It is noted that the conical point leads, rather than follows, the propagation, as is the case for an unperturbed $P$ mode. As the frequency is lowered still further, the propagation again becomes spherical (see also Figure 11). The slowness surfaces for $y=1.1,1.2,1.3$, and 1.5 are shown in Figure 11, and the corresponding surfaces of constant phase in Figure 12. The $\mathbf{P}$ propagation for $\gamma=1.1$ is a wave spreading about the $\mathrm{x}_{3}$ direction. For $\gamma=1.2$ the surface becomes a near-sphere, but flattened in the direction perpendicular to the line of force.

Further insight into the nature of the waves in the lower left-hand corner of the diagnostic diagram is obtained by examining the relative variations of particle and

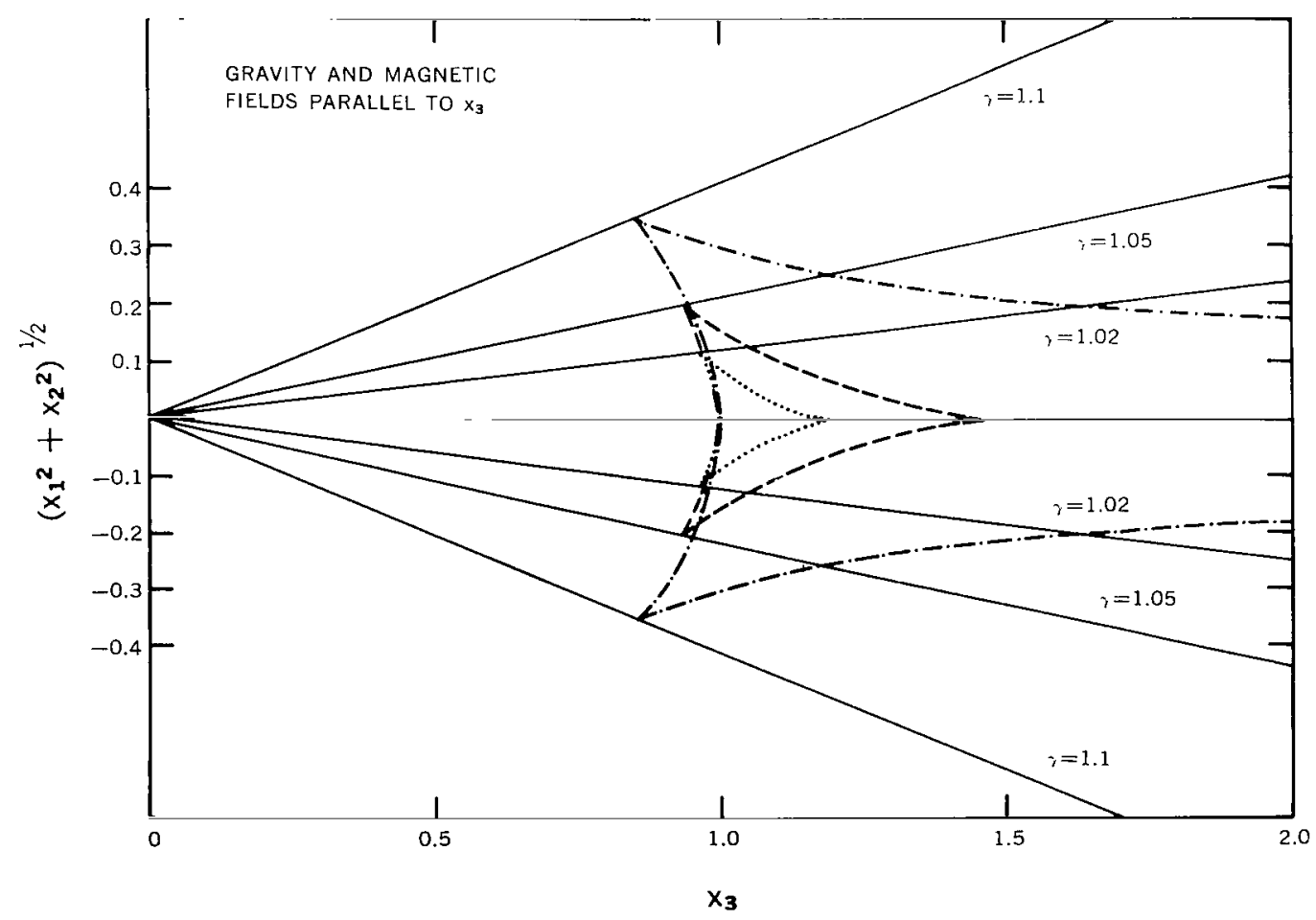

Figure 10 - Constant phase surfaces corresponding to the slowness surfaces of Figure 9. The complete surface is obtained by rotating about the line $\left(x_{1}^{2}+x_{2}^{2}\right)^{\frac{1}{2}}=0$ and across the plane $x_{3}=0$. The conical point leads the propagation. 
magnetic pressure. Combining the equations in entropy, pressure, and $v_{3}$, we have

$$
\left[l^{2}\left(1-\gamma^{2}\right)-\beta^{2} \mathrm{k}_{3}^{2}\right] \mathrm{p}=\left(1-\alpha^{2}\right) l^{2} \beta^{2} \mathrm{p}_{\mathrm{m}}
$$

As an example, consider propagation for the frequency corresponding to $\gamma=1.0$ (see Figure 8). Over most of the slowness surface $k_{3}=l$ and $p+\left(1-\alpha^{2}\right) p_{m}=0$.

An increase in particle pressure results in a

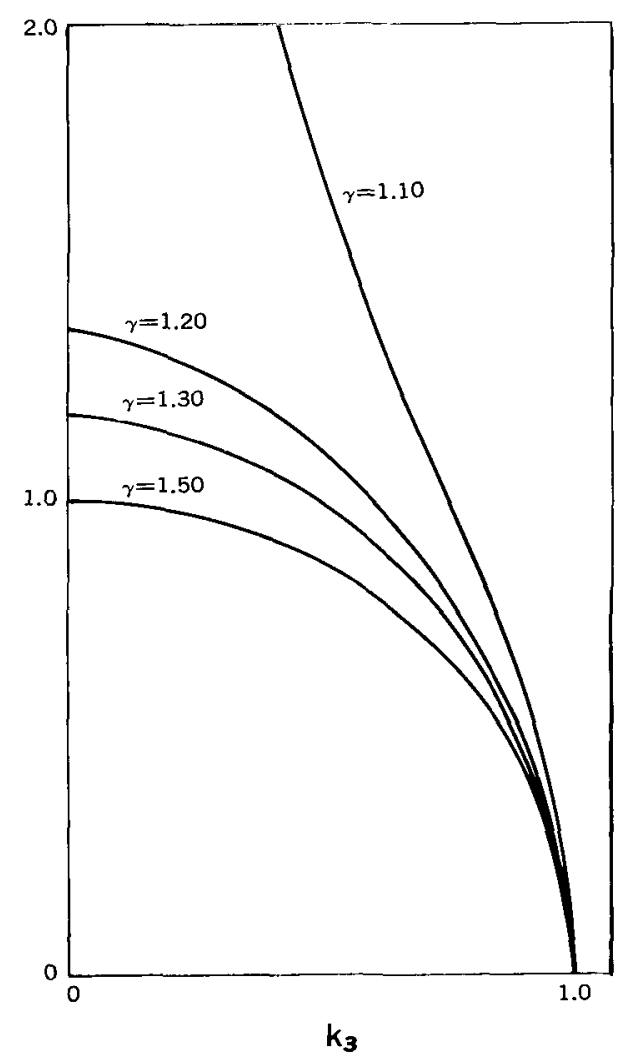

Figure 11 - Slowness surfaces in the lower crosshatched region of Figure 5 . decrease in local magnetic pressure provided that $a<1$. The balance is similar to the balance that exists in a $P$ wave but is modified by the presence of the coefficient multiplying the magnetic pressure. At lower frequencies the propagation approaches that of a $\mathrm{T}$ mode. Thus, for $y=1.5$ the propagation along the direction $k_{3}=0$ gives

$$
\left(1-\gamma^{2} p\right)=\left(1-a^{2}\right) \beta^{2} p_{m}
$$

while the propagation along the direction $k=0$ gives

$$
\left[\left(1-\gamma^{2}\right)-\beta^{2}\right] \mathrm{p}=\left(1-\alpha^{2}\right) \beta^{2} \mathrm{p}_{\mathrm{m}}
$$

The propagation is intermediate between a $\mathrm{T}$ mode and a $\mathrm{P}$ mode, with changes in particle pressure being balanced in part by alterations of the magnetic pressure.

The foregoing arguments show the importance of the frequency $\mathrm{N}_{1}$ in separating those waves that propagate as $\mathrm{T}$ or $\mathrm{P}$ modes from those waves that propagate as modes highly modified by gravity.

The frequency $\mathrm{N}$ is of more fundamental character from a particle point of view, since it is the oscillation frequency of a parcel of charged particles. A further interpretation of $\mathrm{N}$ results from examining the phase relation between the variations in pressure and en tropy. The entropy and pressure equations combine as

$$
Q=\frac{1}{\rho_{0}}\left(\frac{i k_{3}+\frac{g}{C_{0}^{2}}}{N^{2}-\omega^{2}}\right) p,
$$




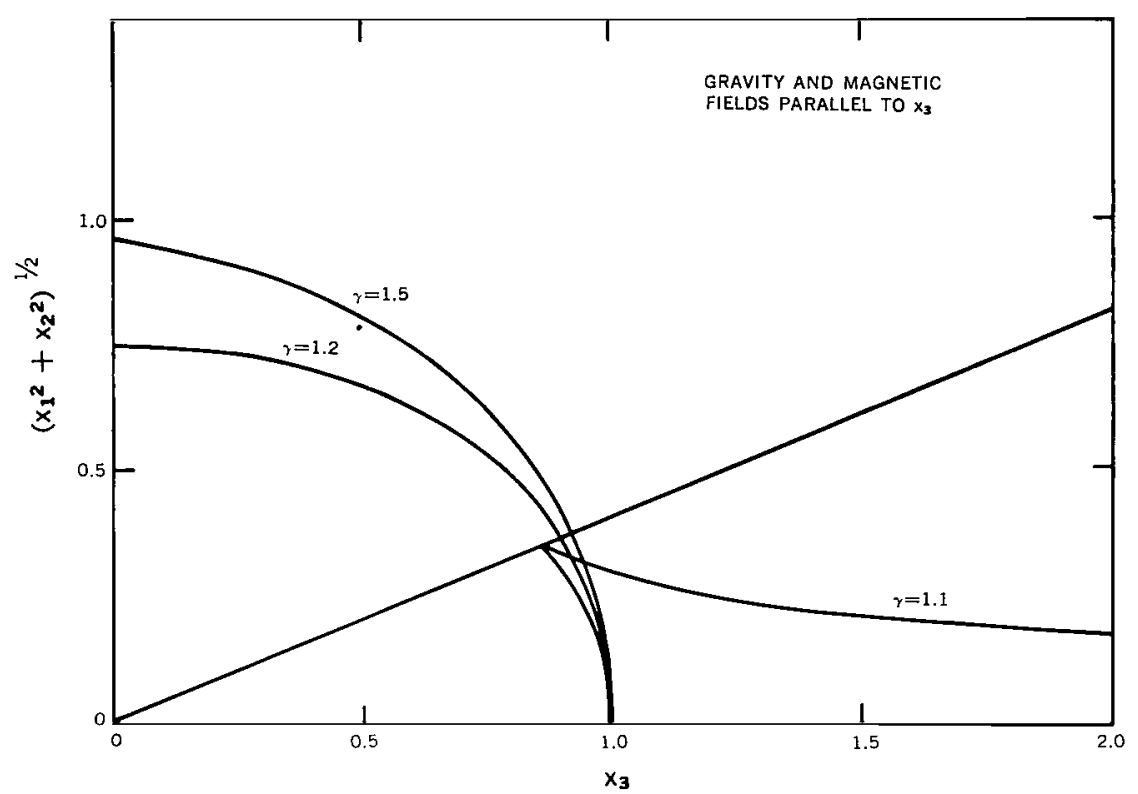

Figure 12 - Constant phase surfaces corresponding to the slowness surfaces of Figure 11.

where $\mathrm{p}$ and $\mathrm{Q}$ are complex numbers with a relative phase given by $\tan ^{-1}\left(\mathrm{k}_{3} \mathrm{C}_{0}^{2} / \mathrm{g}\right)$. The variation in entropy leads the variation in pressure for $\omega<\mathrm{N}$, and the reverse is true for $\omega>N$.

The J.W.K.B. approximation gives a qualitative picture of wave propagation where the density of the medium varies because of the presence of the gravitational field. The parameters $\mathrm{C}_{\mathrm{A}}, \mathrm{C}_{0}, \mathrm{~N}$, and $\mathrm{N}_{1}$ that appear in the dispersion relation will be functions of position, so that the dispersion relation is also a function of the vertical coordinate. The waveform calculated is an approximation for a time that is short compared with the scale length divided by the group velocity. These considerations indicate the importance of the parameters $\mathrm{N}$ and $\mathrm{N}_{1}$ to the propagation of low frequency hydromagnetic waves. The propagation is dispersive at these low frequencies. At frequencies less than $\omega_{2}$ a new mode appears, incorporating some of the features of the $\mathbf{T}$ mode and the $\mathbf{P}$ mode, and bearing some resemblance to the classical gravity wave (Reference 21). 


\section{PART III}

\section{PERPENDICULAR MAGNETIC AND GRAVITATIONAL FIELDS}

The effect of a gravity field is to couple the $\mathrm{P}$ and $\mathrm{T}$ modes at low frequencies. If the magnetic and gravitational fields are parallel, then one mode is still separable, provided new variables - vorticity, and two-dimensional divergence - are chosen; no new choice of variables leads to decoupling in the general case. Once the gravitational and magnetic fields are at an angle, the $\mathrm{V}$ mode is no longer separable. In this case, the vorticity about the line of force involves a component of velocity along the direction of the gravity, and this in turn implies pressure and entropy variations.. The transverse mode also involves a component of velocity along the gravitational field. A further complication is that the axial symmetry is lost, and the slowness surfaces are triaxial.

The effect of a gravity field at an arbitrary angle to the line of force is studied by taking the lines of force parallel to $\mathrm{x}_{1}$ and $\mathrm{g}\left(\partial \mathrm{z} / \partial \mathrm{x}_{1}\right)$ parallel to $\mathrm{x}_{3}$. The distribution of density is assumed to be the result of gravitational forces only. The relevant equations are then

$$
\left.\begin{array}{c}
\frac{\partial \mathrm{b}_{1}}{\partial \mathrm{t}}=-\frac{\partial \mathrm{v}_{2}}{\partial \mathrm{x}_{2}}-\frac{\partial \mathrm{v}_{3}}{\partial \mathrm{x}_{3}}, \\
\frac{\partial \mathrm{b}_{2}}{\partial \mathrm{t}}=\frac{\partial \mathrm{v}_{2}}{\partial \mathrm{x}_{1}}, \\
\frac{\partial \mathrm{b}_{3}}{\partial \mathrm{t}}=\frac{\partial \mathrm{v}_{3}}{\partial \mathrm{x}_{1}} ;
\end{array}\right\}
$$




$$
\begin{aligned}
\frac{\partial \mathrm{p}}{\partial \mathrm{t}}-\mathrm{g} \rho_{0} \mathrm{v}_{3}+\rho_{0} \mathrm{C}_{0}{ }^{2}\left(\frac{\partial \mathrm{v}_{1}}{\partial \mathrm{x}_{2}}+\frac{\partial \mathrm{v}_{2}}{\partial \mathrm{x}_{2}}+\frac{\partial \mathrm{v}_{3}}{\partial \mathrm{x}_{3}}\right) & =0 ; \\
\frac{\partial \mathrm{Q}}{\partial \mathrm{t}}+\mathrm{v}_{3} & =0 .
\end{aligned}
$$

Before considering the dispersion relation for the complete set of equations, Equations 25 through 28 are examined under the approximations $\beta \ll 1$ and $\alpha \approx \gamma$. The dispersion relations give

$$
\begin{aligned}
& \mathrm{k}_{3}^{2}=\frac{\left(\omega^{2}-\mathrm{N}^{2}-\mathrm{C}_{\mathrm{A}}^{2} \mathrm{k}_{1}^{2}\right)\left[\omega^{2}-\mathrm{C}_{\mathrm{A}}{ }^{2}\left(\mathrm{k}_{1}{ }^{2}+\mathrm{k}_{2}{ }^{2}\right)\right]}{\mathrm{C}_{\mathrm{A}}{ }^{2}\left(\omega^{2}-\mathrm{C}_{\mathrm{A}}{ }^{2} \mathrm{k}_{1}^{2}\right)}, \\
& \mathrm{k}_{2}^{2}=\frac{\left(\omega^{2}-\mathrm{C}_{\mathrm{A}}{ }^{2} \mathrm{k}_{1}{ }^{2}\right)\left[\omega^{2}-\mathrm{N}^{2}-\mathrm{C}_{\mathrm{A}}{ }^{2}\left(\mathrm{k}_{1}{ }^{2}+\mathrm{k}_{2}{ }^{2}\right)\right]}{\mathrm{C}_{\mathrm{A}}{ }^{2}\left(\omega^{2}-\mathrm{N}^{2}-\mathrm{C}_{\mathrm{A}} \mathrm{k}_{1}{ }^{2}\right)},
\end{aligned}
$$

where the loss of symmetry is evident. We note that for $\omega \gg \mathrm{N}$ the propagation is a spherically spreading Alfvén wave.

Since the propagation is asymmetric, it is useful to consider the diagnostic diagram in two projections. Figure 13 illustrates the $\omega-k_{1}$ projection (Equation 29) of the diagnostic diagram. The shaded regions correspond to those regions in which $k_{3}{ }^{2}>0$ for a fixed value of $k_{2}$. The lower region is bounded by the line $\omega=\mathrm{C}_{\mathrm{A}} \mathrm{k}_{1}$. The upper boundary is given by

$$
\omega^{2}=\mathrm{C}_{\mathrm{A}}^{2}\left(\mathrm{k}_{2}^{2}+\mathrm{k}_{1}^{2}\right)
$$

provided that

$$
\mathrm{k}_{2}<\frac{\mathrm{N}}{\mathrm{C}_{\mathrm{A}}}
$$

At large values of the wave number $k_{1}$, the upper boundary of the lower region of allowed propagation asymptotically approaches the line $\omega=\mathrm{C}_{\mathrm{A}} \mathrm{k}_{1}$. This line corresponds to a onedimensional Alfvén wave propagation. In the lower shaded region, $k_{3}$ varies from zero to infinity, and in this region the perturbed $V$ mode spreads out about the line of force because of the action of

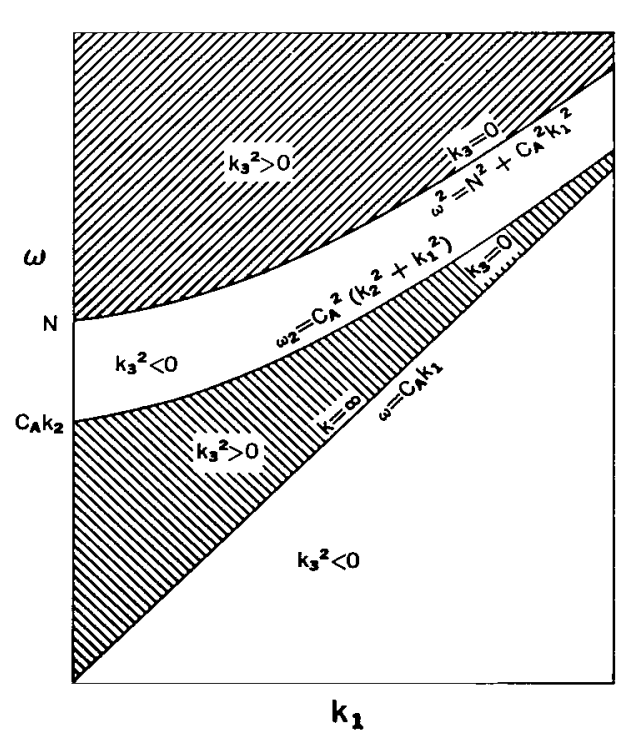

Figure 13-Diagnostic diagram for $\beta=0$; the gravity and magnetic fields are at right angles. The $\omega-k_{1}$ projection is at a fixed value $\mathrm{k}_{2}$ with $\mathrm{C}_{A} \mathrm{k}_{2}<\mathrm{N}$. 
gravity. Furthermore, the curvature of the bounding lines indicates that the propagation is dispersive.

The upper region is bounded by the curve

$$
\omega^{2}=\mathrm{N}^{2}+\mathrm{C}_{\mathrm{A}}^{2} \mathrm{k}_{1}^{2}
$$

This curve asymptotically approaches $\omega=\mathrm{C}_{\mathrm{A}} \mathrm{k}_{1}$ at large values of $\mathrm{k}_{1}$. The propagation in this region corresponds to a perturbed $T$ mode. If $\mathrm{k}_{2}>\mathrm{N} / \mathrm{C}_{\mathrm{A}}$, the diagnostic diagram takes the for $m$ given in Figure 14. The lower region of allowed propagation is divided into two at $\omega=\mathrm{N}$. Its upper boundary is given by Equation 32 and its lower boundary by Equation 31. As in the case of $k_{2}<N / C_{A}$, the bounding curves asymptotically approach $\omega=\mathrm{C}_{\mathrm{A}} \mathrm{k}_{1}$.

The diagnostic diagram corresponding to Equation 30 is shown in Figure 15. The parameter $k_{3}$ is taken as fixed parameter and the allowed regions in the $\omega-k_{1}-k_{2}$ space are projected. The $k_{2}$ parameter becomes very large along the line given by Equation 32 , and vanishes along the line $\omega=\mathrm{C}_{\mathrm{A}} \mathrm{k}_{1}$ and along the curve fixed by the equation

$$
\omega^{2}=\mathrm{N}^{2}+\mathrm{C}_{\mathrm{A}}^{2}\left(\mathrm{k}_{1}^{2}+\mathrm{k}_{3}^{2}\right)
$$

The propagation in the upper region will be nearly spherical, while the propagation in the lower region corresponds to a one-dimensional wave spreading about the line of force. As $\kappa_{s}$ becomes small, the lower region of allowed propagation shrinks to a double valued boundary line.

The representation of the slowness surfaces corresponding to the diagnostic diagrams Figures 13 through 15 is difficult because of the essential three-dimensional character of the propagation. Furthermore, the representation of the constant phase surfaces with several cuspidal edges is not easily managed. The equation of the slowness surface is

$$
\mathrm{k}_{1}^{4}+\left[\mathrm{k}_{2}^{2}+\mathrm{k}_{3}^{2}-l^{2}\left(2-a^{2}\right)\right] \mathrm{k}_{1}^{2}+l^{2}\left[l^{2}\left(1-a^{2}\right)-\left(\mathrm{k}_{2}^{2}+\mathrm{k}_{3}^{2}\right)+a^{2} \mathrm{k}_{2}^{2}\right]=0
$$

where $a$ is given by $a=\mathrm{N} / \omega$; and $l$ is given by $l=\omega / \mathrm{C}_{\mathrm{A}}$. Figure 16 gives a schematic representation of the slowness surface for $\mathrm{N} / \omega=0.5$.

The two sheets of the propagation surface are shown in Figures 17 and 18. Figure 17 is a projection of propagation surface (Equation 33) on to the $k_{1}-k_{3}$ plane. The complete surfaces are obtained by reflection across the coordinate planes. The lower region of the diagnostic diagram (Figure 13) collapses into a line for $k_{2}=0$, and the propagation 

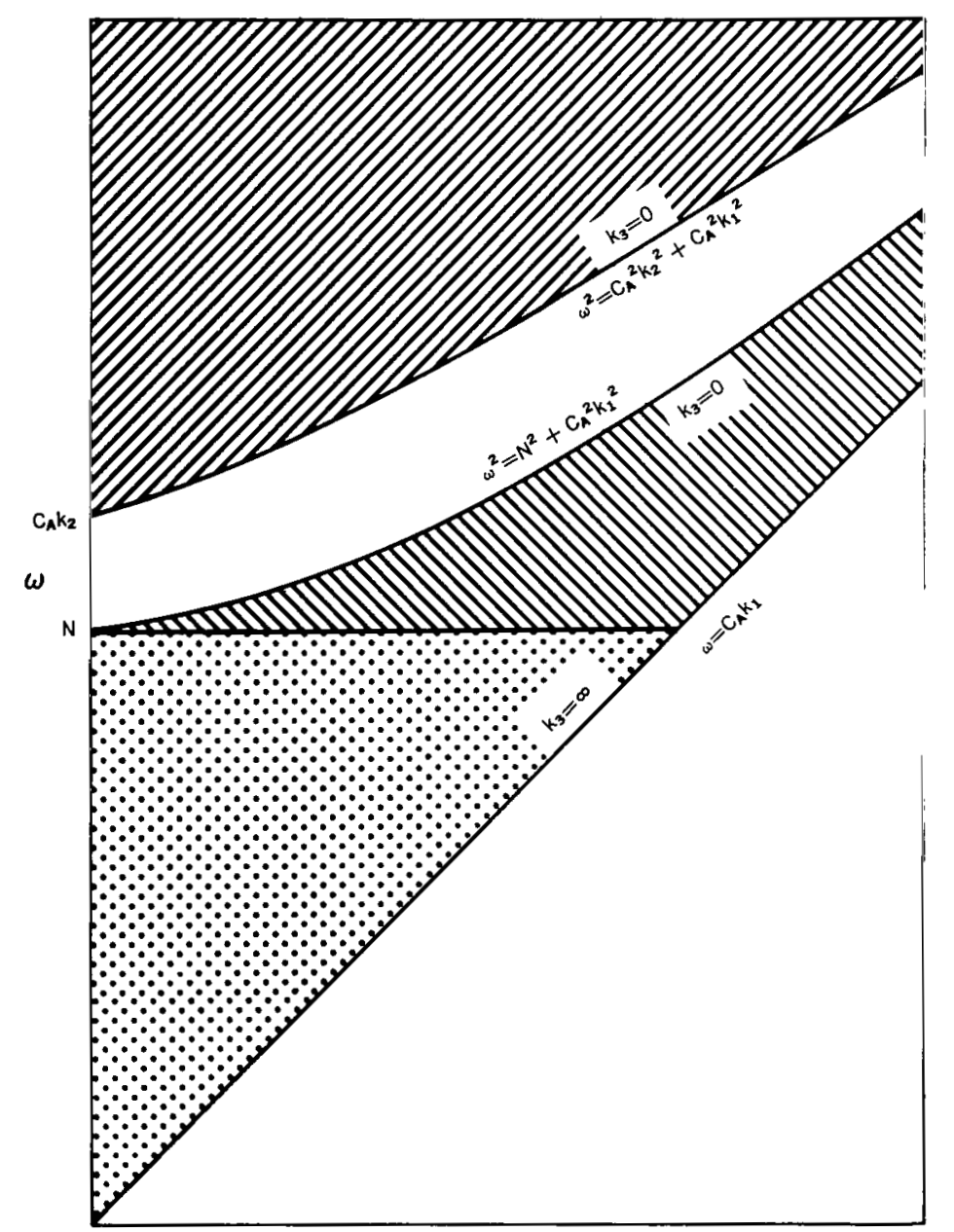

$k_{1}$

Figure 14 - Diagnostic diagram for a fixed value of $k_{2}$ with $\mathrm{C}_{\mathrm{A}} \mathrm{K}_{2}>\mathrm{N}$.

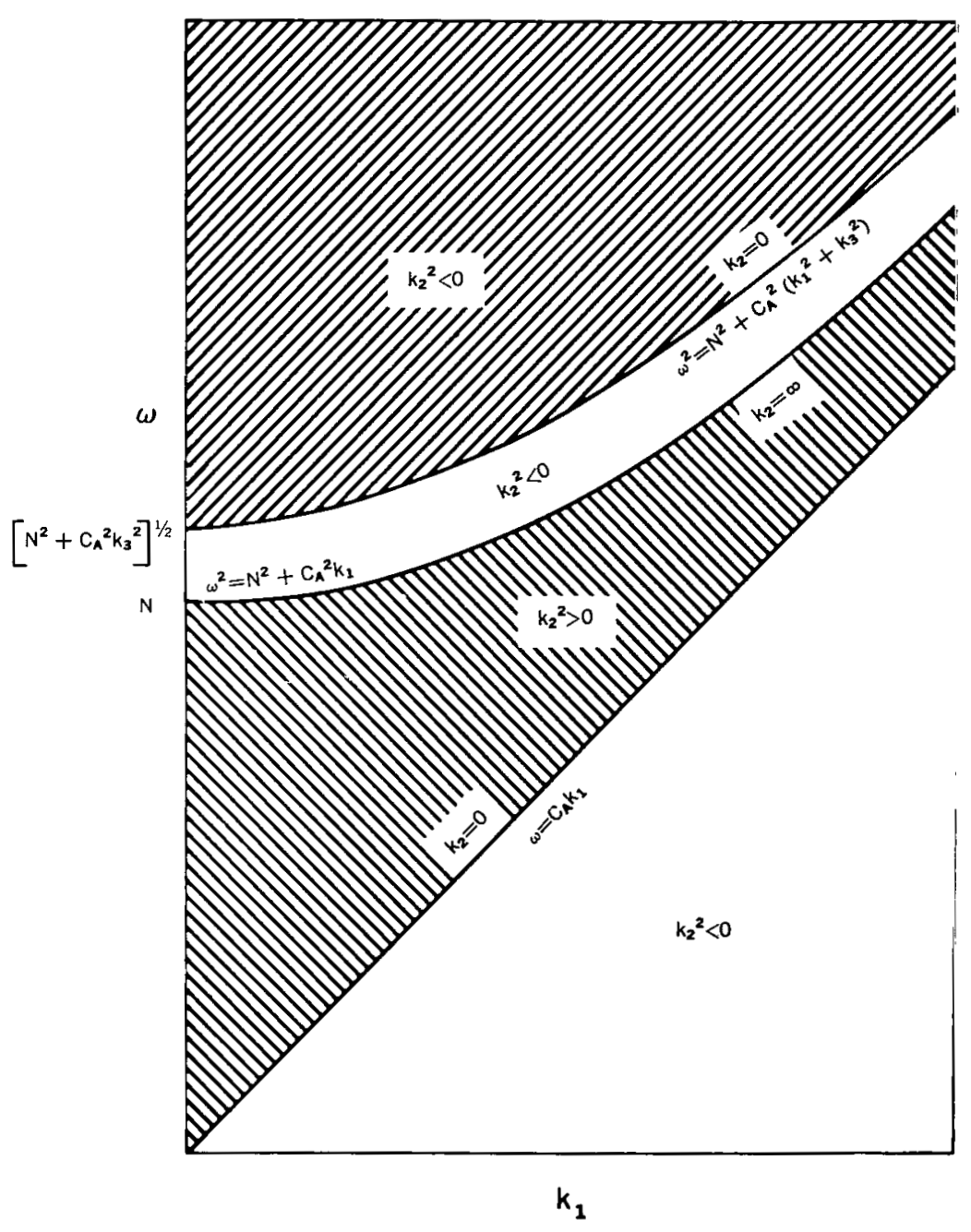

Figure 15 - Diagnostic diagram for a perpendicular magnetic and gravity field at a fixed value of $k_{3}$.

$\underset{\omega}{\infty}$ 


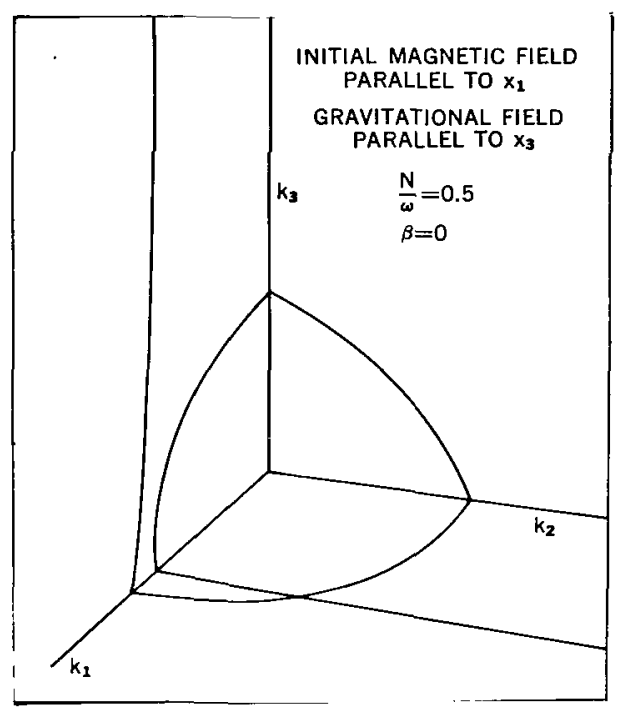

Figure 16 - Slowness surfaces for perpendicular gravity and magnetic field at $\beta=0$. A complete surface is obtained by reflection across the coordinate planes. of the $\mathrm{V}$ mode is one-dimensional at all frequencies. The upper region corresponds to a spherically spreading mode, a perturbed $T$ mode. The $\mathrm{k}_{2}=0.5$ cut shows that the $\mathrm{V}$ mode has now spread about the $k_{1}$ axis; there is a parabolic point in this plane and a corresponding cuspidal edge in the phase surface. The $\mathrm{T}$ mode is a triaxial ellipsoid, as is evidenced from the $k_{2}=0.5$ and 0.9 cuts through the phase surface. As $k_{2}$ becomes larger, the $\mathrm{V}$ mode slowness surface shows a greater curvature.

Figure 18 is the projection of the slowness surface onto the $k_{1}-k_{2}$ plane. For large values of $k_{3}$ the propagation becomes nearly one-dimensional along the $k_{1}$ axis and there is no $T$ mode. Note that at $k_{3}=0$, the two sheets of the slowness surface intersect (see Figures 15 and 16).

At lower frequencies $(\omega<N)$ there is only a single propagating mode. The projection of the slowness surface on the $k_{1}-k_{3}$ plane is shown in Figure 19. The propagation is one-dimensional at Alfvén wave velocity along the line of force for $k_{2}=0$. At higher values of $k_{2}$ the propagation spreads about the $\kappa_{1}$ axis, so that for large values of $k_{2}$ the propagation is along the $x_{3}$ axis. In the presence of a gravitational field, the propagation at very low frequencies takes the form of waves spreading about the direction perpendicular to the lines of force. The $k_{2}-k_{3}$ plane cuts the slowness surface along a hyperbola, while the $k_{1}-k_{2}$ projection is a modified circle.

The dispersion relation for finite $\beta$ is

$$
\begin{aligned}
0=\beta^{2} \mathrm{k}_{1}{ }^{6} & +\mathrm{k}_{1}{ }^{4}\left\{\beta^{2}\left[\mathrm{k}_{2}{ }^{2}+\mathrm{k}_{3}{ }^{2}-\left(2-a^{2}\right) l^{2}\right]-l^{2}\right\} \\
& +\mathrm{k}_{1}{ }^{2}+\left(\beta^{2}\left\{l^{4}-l^{2}\left[a^{2}\left(l^{2}-\mathrm{k}_{2}{ }^{2}\right)+2\left(\mathrm{k}_{3}{ }^{2}+\mathrm{k}_{2}\right)\right]\right\}\right. \\
& \left.+l^{4}\left(2-\gamma^{2}\right)-l^{2}\left(\mathrm{k}_{3}{ }^{2}+\mathrm{k}_{2}{ }^{2}\right)\right) \\
& +\beta^{2} l^{4}\left(\mathrm{k}_{3}{ }^{2}+\mathrm{k}_{2}{ }^{2}-\alpha^{2} \mathrm{k}_{2}{ }^{2}\right) \\
& +l^{4}\left[\mathrm{k}_{3}{ }^{2}+\mathrm{k}_{2}{ }^{2}\left(1-\gamma^{2}\right)+l^{6}\left(\gamma^{2}-1\right)\right] .
\end{aligned}
$$




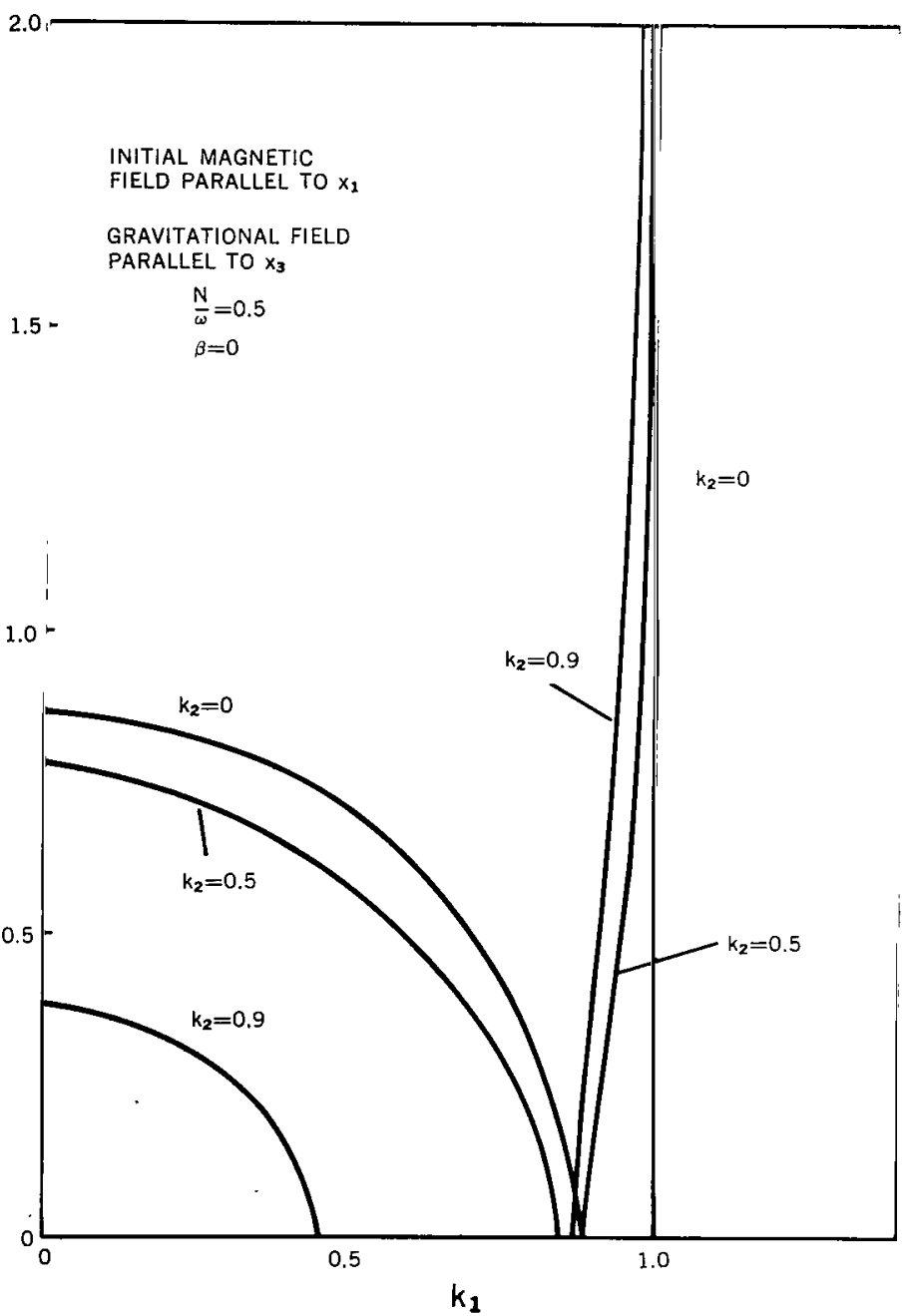

Figure 17 - The slowness surface for $1 / \omega=0.5$. The surfaces are projected onto $k_{1}-k_{3}$ plane. The near-plane corresponds to perturbed $V$ mode propagation, the sphere to $T$ mode propagation.

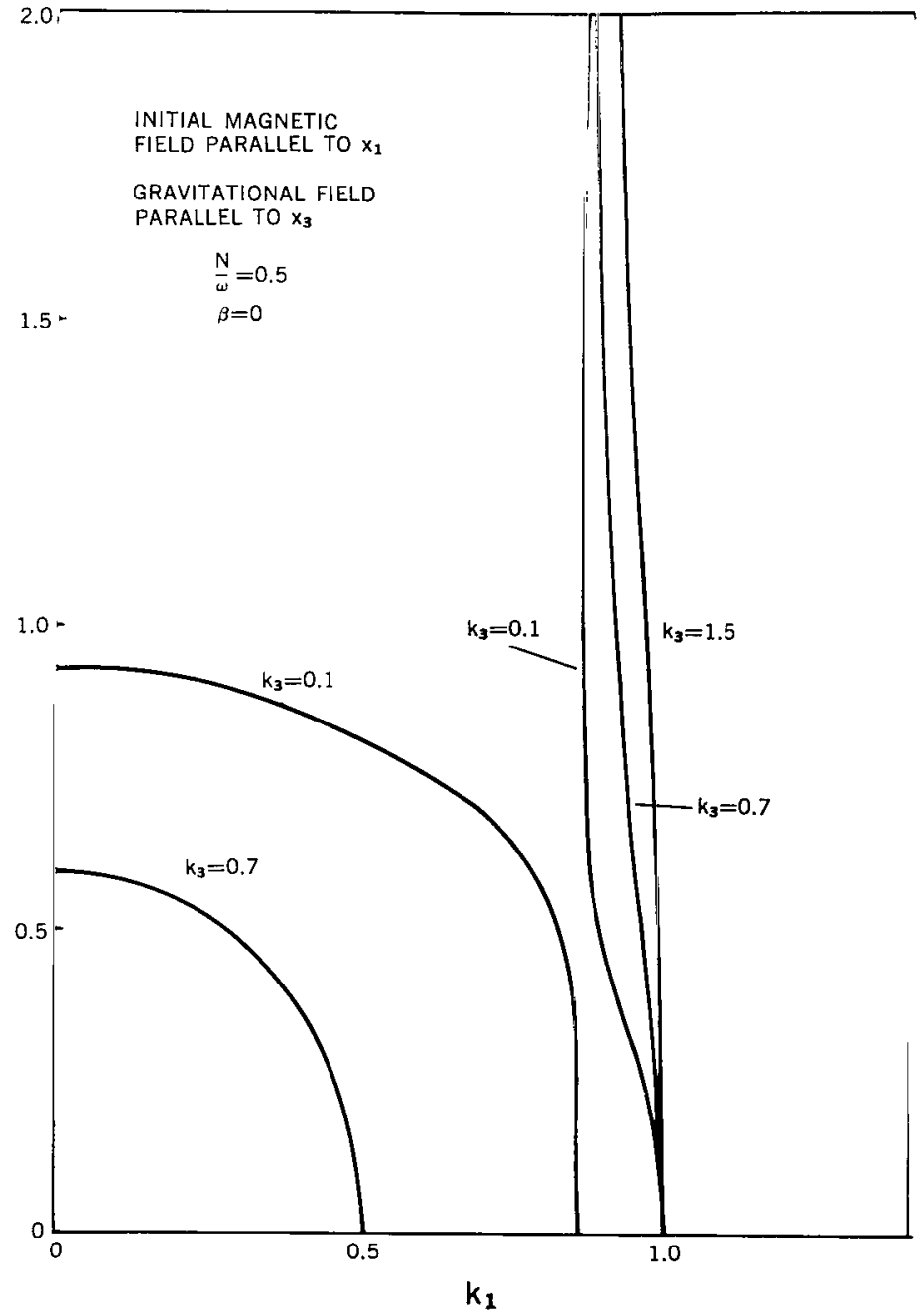

Figure 18 - The slowness surfaces of figure 17 shown on a $k_{1}-k_{2}$ projection 


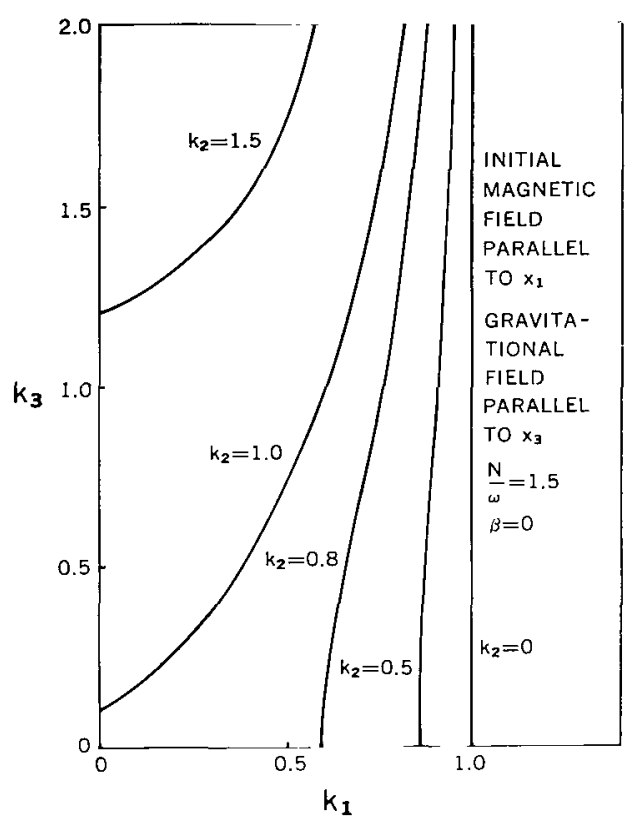

Figure 19 - Slowness surface for propagation at $N / \omega=1.5$. The threedimensional slowness surface is an asymmetric hyperbola.

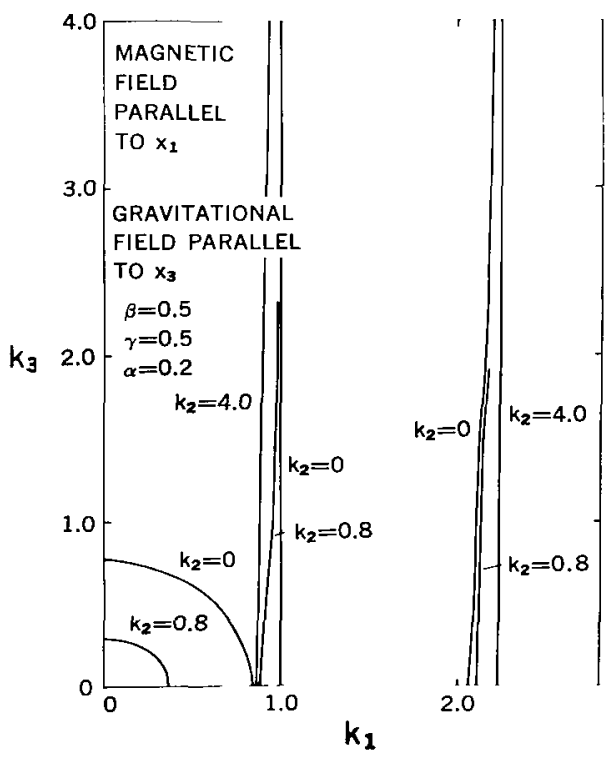

Figure 20 - Slowness surface for a perpendicular gravitational and magnetic field at $\beta=0.5$. The slowness surfaces consist of three sheets corresponding to the perturbed $P, V$, and T modes.
The corresponding slowness surfaces show features similar to both those of Figures 6 and 8 and of Figures 17, 18, and 19. The slowness surfaces are composed of three sheets: two corresponding to nearly one-dimensional propagation, and a third, a triaxial ellipsoid, corresponding to the propagation of the gravitationally perturbed $\mathrm{T}$ mode. The projections on the $k_{1}-k_{3}$ plane of the sheets for the case of $\beta=0.5$ and $\gamma=0.5$ is shown in Figure 20. The projection at large values of $k_{2}$ shows the two nearly one-dimensional $\mathrm{P}$ and $\mathrm{V}$ mode propagations. The $\mathrm{V}$ mode propagation for $\mathrm{k}_{2}=0$ is strictly one-dimensional along the direction of the magnetic field. The slowness surfaces show curves of parabolic points both in the $\mathrm{V}$ and $\mathrm{P}$ modes, and these transform into cuspidal edges in the constant phase surfaces.

The transition between the perturbed $\mathrm{P}-\mathrm{V}-\mathrm{T}$ modes and the gravitational modes for finite $\beta$ is shown in Figure 21. For $\gamma=0.9$ the triaxial ellipsoid representing the perturbed $\mathrm{T}$ mode has shrunk to a surface about the origin; the corresponding phase velocity is higher than in the absence of gravity. The phase velocity of the perturbed $\mathrm{V}$ mode in the $\mathrm{k}_{1}$ direction increases with increasing $\gamma$ while the phase velocity of the perturbed $\mathbf{P}$ mode decreases.

At the transition frequency $(y=1.0)$ there is no propagation in the $\mathrm{T}$ mode and only the one-dimensional highly perturbed $\mathrm{V}$ and $\mathrm{P}$ modes remain. The slowness surface for the $\mathrm{P}$ mode remains very nearly a plane at all values of $k_{2}$, but the $\mathrm{V}$ mode propagation undergoes spreading at intermediate values of $k_{2}$. As the frequency decreases, the $\mathrm{V}$ mode is propagated along the direction perpendicular to the lines of force, while the much perturbed acoustic mode ( $P$ mode) remains one-dimensional along the direction of the magnetic field. 
Gravity renders the propagation dispersive. In the presence of perpendicular magnetic and gravitational fields the constant phase surfaces corresponding to the gravitationally perturbed $\mathrm{P}$ and $\mathrm{V}$ modes show cuspidal edges corresponding to the curves of parabolic points on the slowness surfaces. The disturbance tends to be concentrated at the cuspidal edges and the conical point, so that a planar projection of the disturbance would show a disturbance in the form of an ellipse, with a central point. The dispersion is such that for frequencies less than $\mathrm{N}_{1}$, the lower frequency components travel at a greater phase velocity than the higher frequency components. The effect of gravity in the case of both parallel and perpendicular gravity and magnetic fields is to couple the modes in a complicated way at frequencies less than the critical gravitational frequency $\mathrm{N}_{\mathbf{1}}$. 


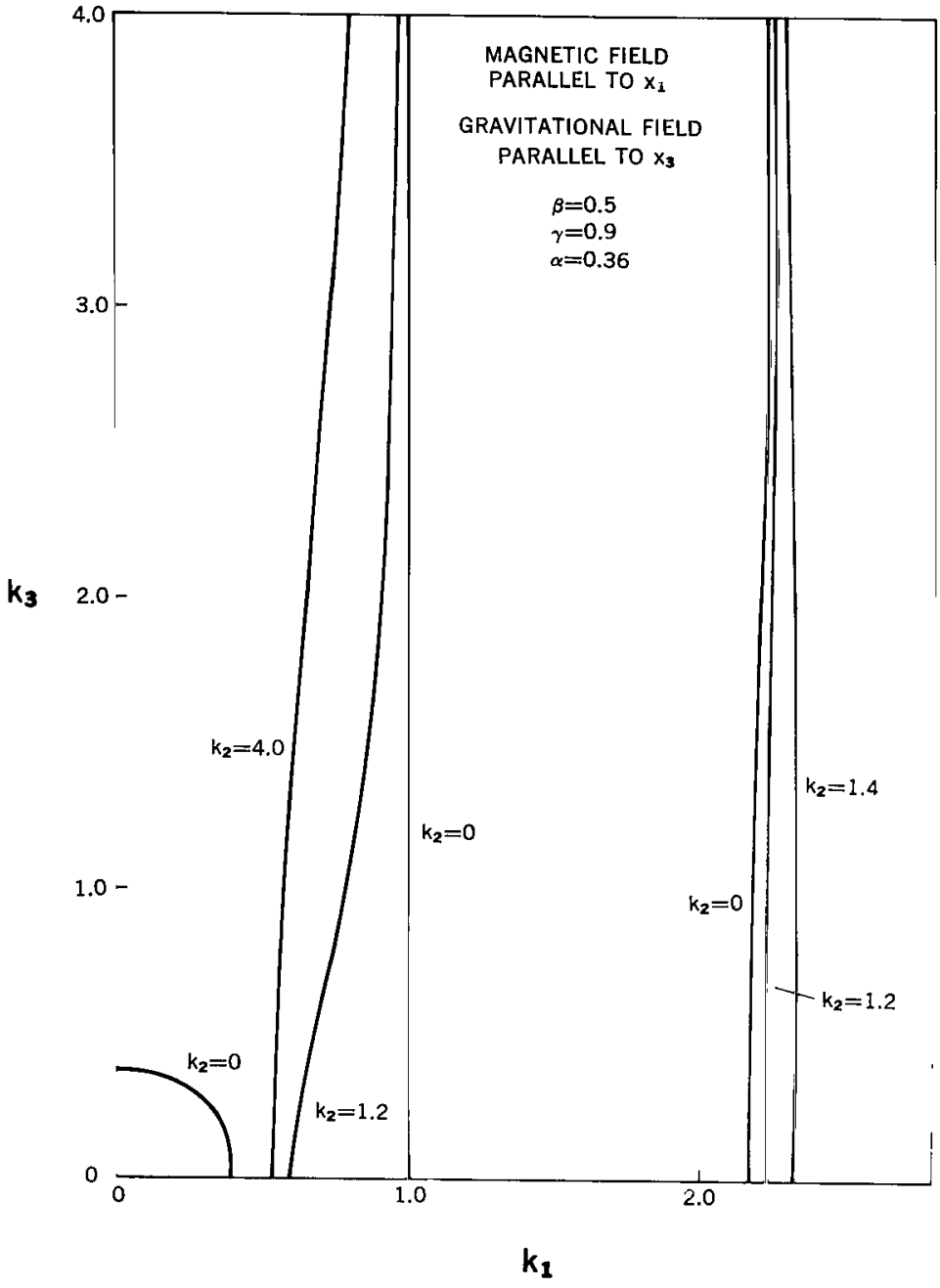

(A)

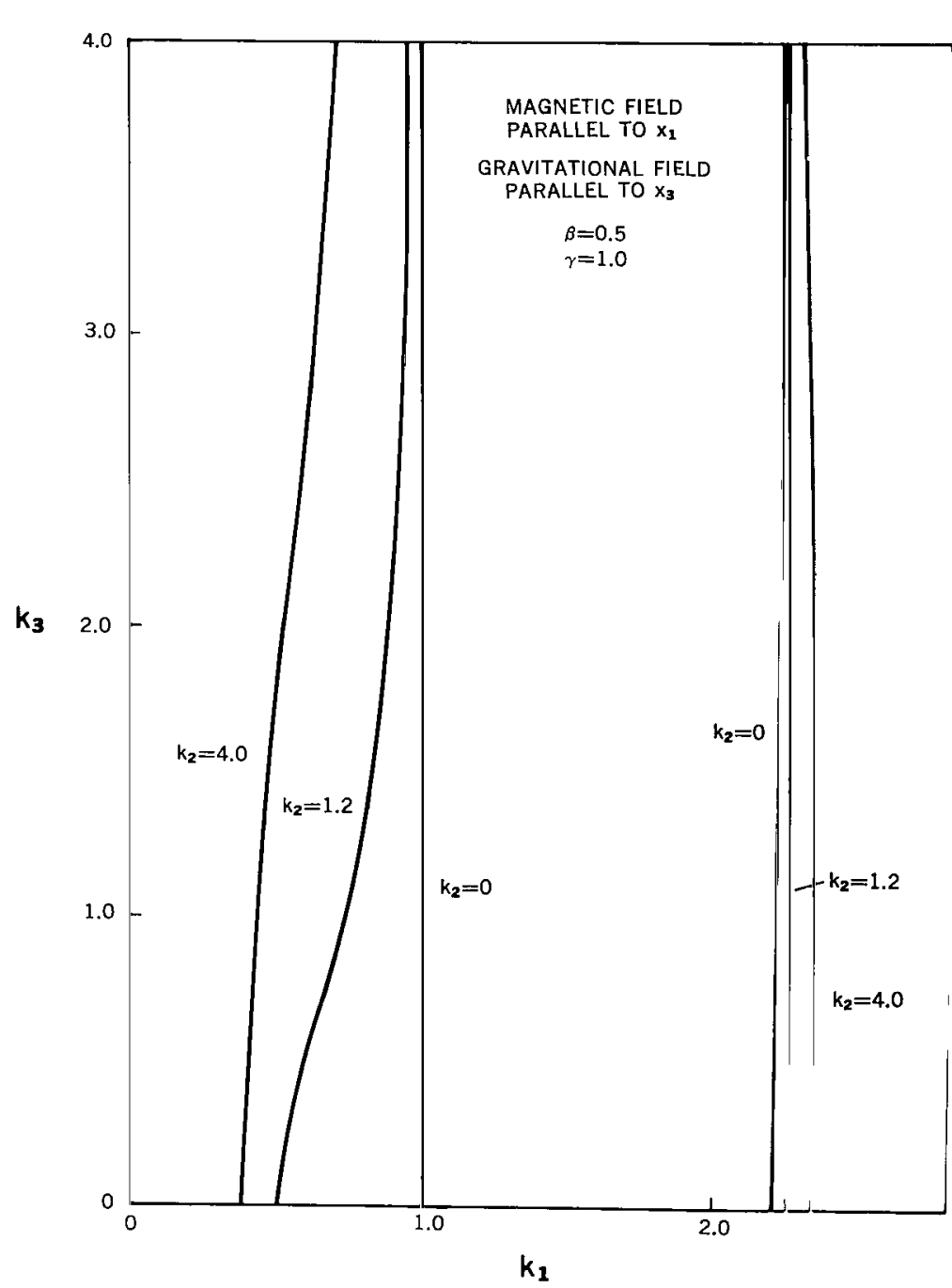

(B)

Figure 21 - Slowness surfaces for frequencies near the critical frequency $N_{1}$ for perpendicular gravity and magnetic fields 


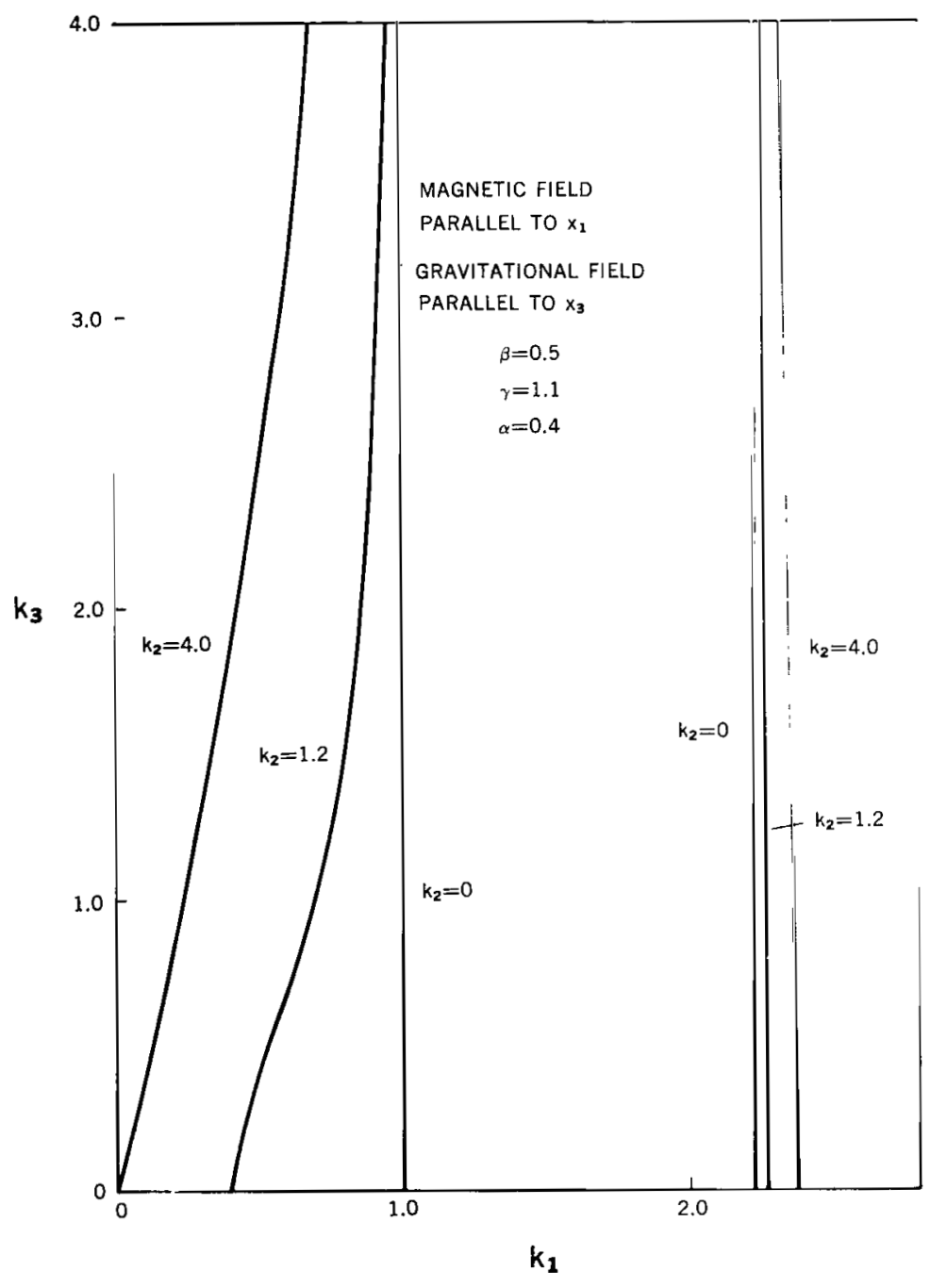

(C)

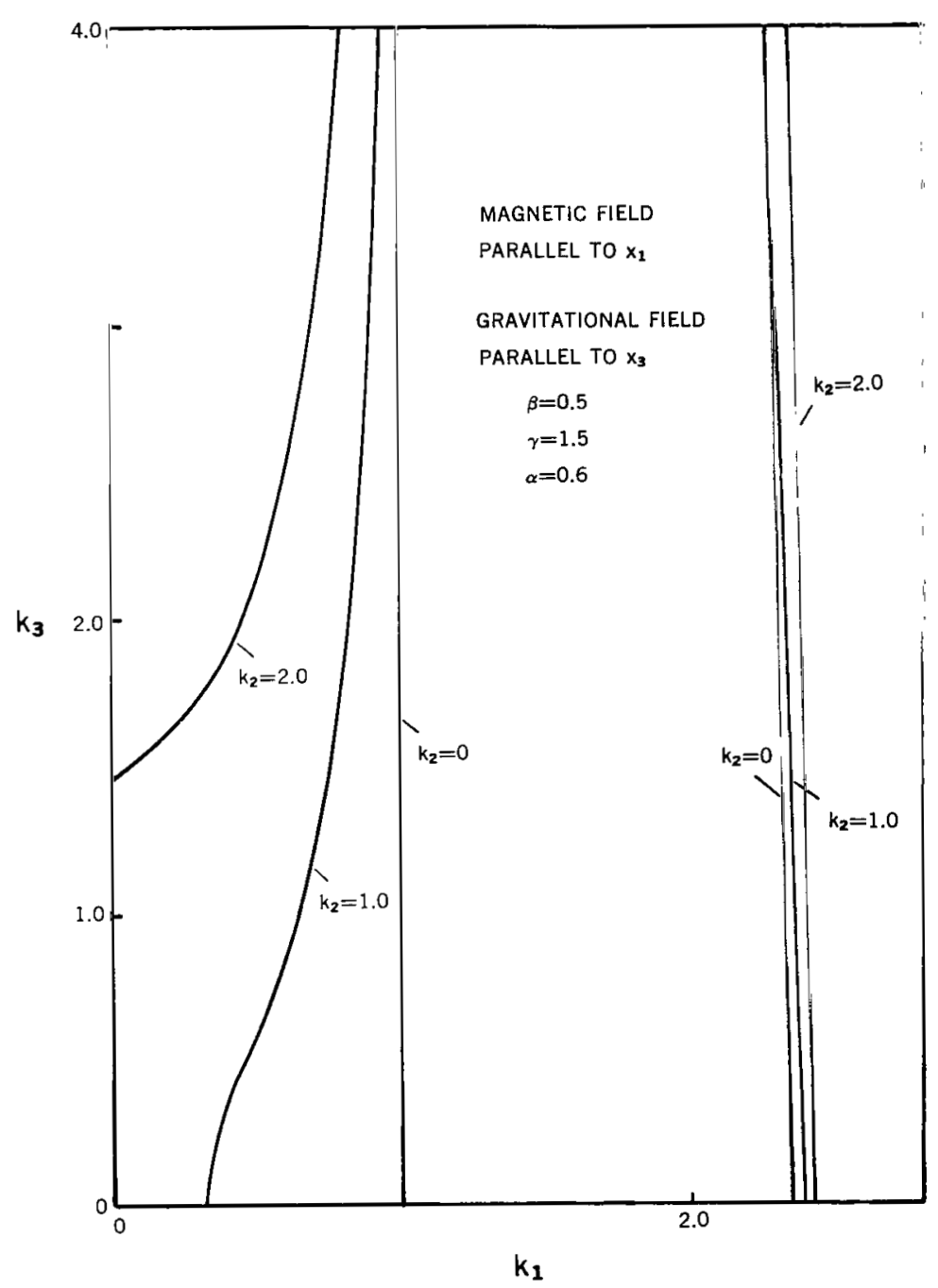

(D)

Figure 21 (Cont.) - Slowness surfaces for frequencies near the critical frequency $N_{1}$ for perpendicular gravity and magnetic fields 


\section{PART IV}

\section{CONTINUUM APPROXIMATIONS}

The exosphere is a low density, partially ionized gas. A description of waves in the exosphere by the equations of magnetohydrodynamics implies that the gas can be treated as a continuum. In a rarified neutral gas the continuum equations would apply, provided that the length scales of the phenomena were large compared with the mean free paths. However, the wide range of physical phenomena involved in an ionized gas does not permit a simple working rule defining the limit of applicability of the continuum equations. The problem is particularly vexing in the exosphere, since conventional criteria indicate that a wide range of processes do not satisfy the conditions of either a continuum or a kinetic theory. An exploration of the problem would require a development of the predictions of the continuum theory and their comparison with experiment. As an example, the continuum theory makes certain suggestions regarding the effect of a satellite on the exosphere. Experimental tests of these predictions would allow an estimate of the applicability of the continuum theory to other phenomena having comparable length scales.

The sound wave velocity $\mathrm{C}_{0}=\sqrt{\gamma_{\mathrm{p}} / \rho}$ is about 1 to $3 \mathrm{~km} / \mathrm{sec}$ in the exosphere. The Alfvén wave velocity $C_{A}$ is much larger, ranging from several hundred to several thousand $\mathrm{km} / \mathrm{sec}$. The ratio $\beta$ of these two velocities is a small number, on the order of $10^{-2}$ to $10^{-3}$. The ratio of the charged particle pressure to the magnetic pressure, $\mathrm{H}^{2} / 8 \pi$, is of the order of $\beta^{2}$. Thus, over the entire exosphere, the magnetic pressure dominates the particle pressure. It should be noted that the usual interpretation of the Alfven velocity as a propagation speed of transverse waves along magnetic force lines in an incompressible fluid requires that the Alfvén wave velocity be much less than the sound wave velocity. When the opposite holds true, then the Alfvén waves must be reinterpreted as one-dimensional propagations of vorticity and the spherical propagations of the transverse components of velocity.

The $\mathbf{P}$ mode involves the propagation of fluctuations in charged particle pressure. It would seem that the characteristic length limiting the continuum approximation is the length over which an appreciable portion of particle momentum is exchanged. The $\mathrm{V}$ and $T$ modes involve the propagation of the transverse components of the velocity. Because of the magnetic field, however, the charged particles spiral about the lines of force. Thus the motion of the charged particles in directions at right angles to the lines of force is greatly restricted, while the motion along the lines of force is largely independent of the magnetic field. It would be expected, then, that the characteristic length 
signifying applicability of the continuum approximations would involve the radius of gyration. The fact that different length scales are involved in the different modes is intimately connected with the anisotropic nature of the wave propagation. Thus, there may be conditions for which $\mathrm{V}$ and $\mathrm{T}$ mode propagation may take place, yet there would be no $\mathbf{P}$ mode propagation.

In a partially ionized gas there are several mean-free paths corresponding to neutral-neutral collisions, neutral-charged particle collisions, etc. Inelastic processes, such as ionization and recombination, introduce further complications. The neutralneutral mean free path is the distance a neutral particle must travel before it exchanges its momentum by a direct collision with another neutral particle. The difference between the neutral-neutral mean free path and the neutral-charged particle mean free path is small. Over the entire exosphere the neutral gas mean free path exceeds several kilometers and is large compared to other lengths that characterize the motion of charged particles. It is because of this large difference in the representative length scales of neutral and charged particle gases that the two gases are usually assumed to act independently. However, for large disturbances in the lower exosphere this no longer holds true, and the influence of the neutral molecules on the wave propagation must be considered.

As a result of the Coulomb interactions a charged particle exchanges its momentum, not by single collisions, but in small bits through distant interaction. Because of the small but frequent exchanges of momentum, a particle may transfer an appreciable por tion of its momentum in distances that are short compared to the distance a particle must travel to exchange all of its momentum. In the calculation of the charged particle mean free path (References 30 and 31 ) the angular deflection in the path of the particle traveling at a velocity $\mathrm{V}$ relative to a given particle is computed as a function of the distance between the two. A particle traveling a distance $r$ will, on the average, come to within a distance $s=1 / n_{i} \pi r^{1 / 2}$ where $n_{i}$ is the charged particle density. The loss of directed momentum is obtained by summing up over encounters at different distances of separation. The lower limit of the integration is taken as zero, corresponding to direct but highly improbable collisions, while the upper limit is taken as the Debye length $\sqrt{\mathrm{kT} / 4 \mathrm{~m}_{\mathrm{i}} \mathrm{e}^{2}}$.

The interpretation of the Debye length is the distance over which the removal of all charged particles of one sign would result in an electric field comparable to $\mathrm{kT}$. The Debye length ranges from a few tenths to a few tens of centimeters in the exosphere; this implies that a charged particle can interact with another charged particle at distances of $10^{6}$ to $10^{8}$ particle diameters away. The distance an electron must travel in order to exchange all of its momentum is then given by

$$
l_{\mathrm{m}}=\frac{1}{4 \pi \mathrm{n}_{\mathrm{i}}} \frac{(3 \mathrm{kT})^{2}}{\mathrm{e}^{2}}\left[\ln \left(1+\mathrm{a}^{2}\right)\right]^{-1},
$$


where

$$
\mathrm{a}=\frac{3}{2} \mathrm{ze}^{3} \frac{\left(\mathrm{k}^{3} \mathrm{~T}^{3}\right)^{\frac{1}{2}}}{\pi \mathrm{n}}
$$

Alternatively, the distance $l_{x}$ a particle must travel to exchange a fraction $x$ of its initial momentum is

$$
l_{x}=\frac{1.29 \times 10^{5} \mathrm{~T}^{2}}{\mathrm{n}_{\mathrm{i}} \ln (\mathrm{a}+\mathrm{i})} \mathrm{x}^{2}
$$

where

$$
\mathrm{x}=\frac{\mathrm{v}^{2}}{\overline{\mathrm{v}}_{\mathrm{e}}^{2}}
$$

$v$ being the velocity component perpendicular to the initial motion of the particle and $\bar{v}$ being the thermal velocity. The quantity $\ln (a+1)$ is about 10 to 20 in the exosphere (Reference 31). Thus, the elementary theory of the particle interaction states that the distance a particle must travel to exchange a fraction $\mathrm{x}$ of its momentum depends on the square of the proportional change in momentum.

The interpretation of the $\mathrm{V}$ mode as the propagation of vorticity along the line of force implies that the condition for the continuum approximation to hold is that the disturbance have a large length scale as compared to the root-mean-square of the spiral of the ions about the line of force. The Larmor radius is $\left(k \mathrm{Tm}_{i}\right)^{1 / 2} / \mathrm{eB}$, where $\mathrm{m}_{\mathrm{i}}$ is the mass of the charged particle. We can also interpret the Larmor radius as the length over which the particle pressure $n k T$ is comparable to the magnetic Lorentz force $B n e(k T / m)^{1 / 2}$. In a strong field, a dilute gas has a Larmor radius much smaller than the mean free path. Collisionless hydromagnetic waves are then propagated through the intervention of the external field.

We now consider the applicability of the continuum equations for two models of the exosphere, in both of which the magnetic field is taken to be that of the dipole with an equatorial surface field of 0.315 gauss. Two distributions of charged particle density are considered. The high density model is that proposed by Dessler, Francis, and Parker (Reference 12) and based on the ion density values of Johnson (Reference 32). Johnson has calculated the ion distribution in a gravitational field above the F2 layer assuming diffusive equilibrium. The model gives a maximum ion number density of $1.1 \times 10^{6}$ particles $/ \mathrm{cm}^{3}$ at an altitude of $2.5 \times 10^{4}$ kilometers and 400 particles $/ \mathrm{cm}^{3}$ at an altitude of $2.5 \times 10^{4}$ kilometers. The molecular weight of the lower exosphere is 16 , decreasing rapidly to 1 in the region from 1000 to 2500 kilometers. The assumed 
magnetic field and density distribution combine to yield the variation of Alfvén wave velocity shown as the high density model in Figure 22.

An alternative model of the exosphere has been constructed to indicate the range of possibilities. Hanson and McKibbin (Reference 33) have carried out an ion trap measurement of the ion concentration profile in the region from 240 to about 800 kilometers in an early-evening rocket launching. Above 350 kilometers the concentration profile approximates a hydrostatic distribution of ions of mass 16 at a temperature of $1240^{\circ} \mathrm{K}$. Smith and Helliwell (Reference 34) have obtained an electron density of 100 electrons $/ \mathrm{cm}^{3}$ at 5 earth radii using nose whistlers. This value is in good agreement with the determination of Blackwell and Ingham (Reference 6) of outer coronal densities. The whistler values suggest somewhat lower densities than those used by Dessler, Francis, and Parker. The low density model is based, at large distances, on the whistler data; and in the lower exosphere, on the rapid determinations of Hanson and McKibbin. The hydrostatic calculation at $1300^{\circ} \mathrm{K}$ has been carried out with assumptions regarding the variation of ion composition similar to those used by Johnson, with $1300^{\circ} \mathrm{K}$ as the temperature. The magnetic field and density combine to give the low density model variations of the Alfven wave velocity shown in Figure 22.

The rms Larmor radius depends only on the mean molecular weight, temperature, and magnetic field. For an isothermal exosphere at $1300^{\circ} \mathrm{K}$ the two models lead to similar rms Larmor radii, as is shown in Figure 23. At an altitude of 30,000 kilometers the $\mathrm{rms}$ Larmor radius is about 100 meters.

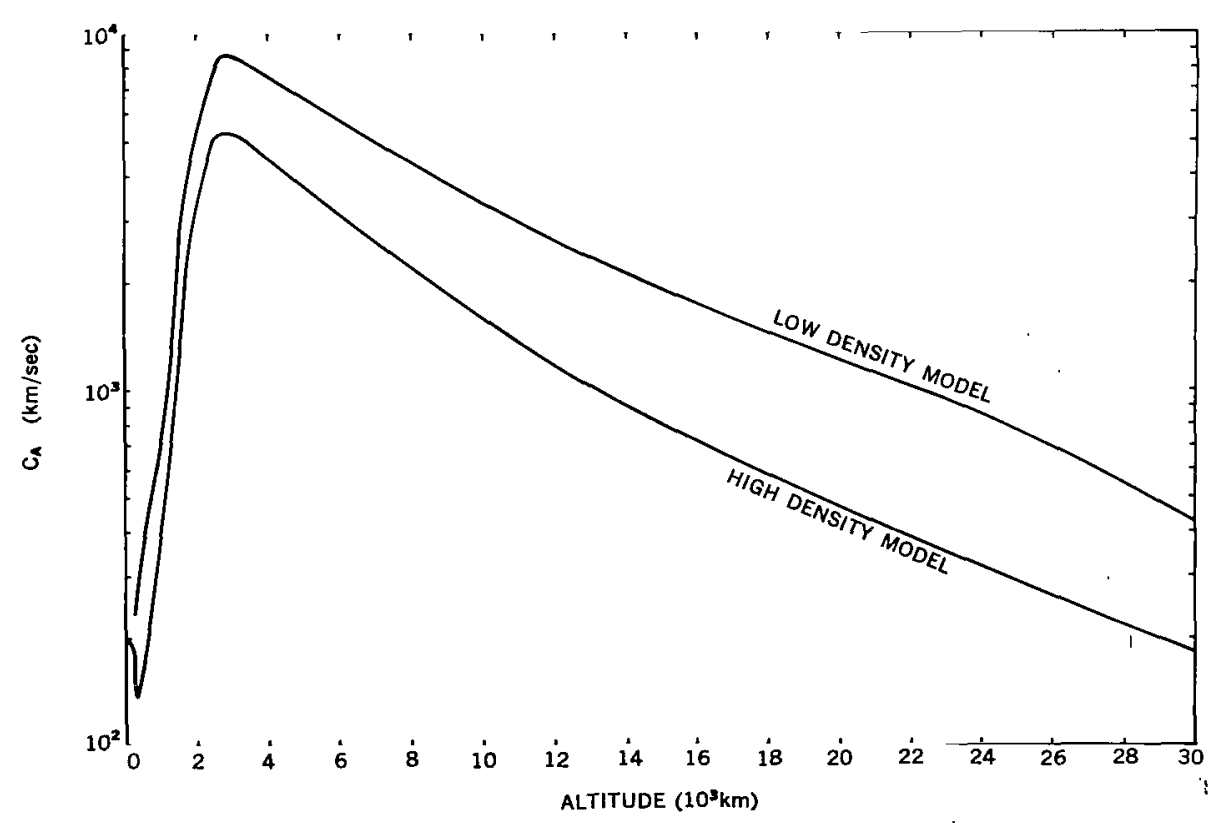

Figure 22 - The variation of equatorial Alfvén wave velocity for two models of the exosphere 


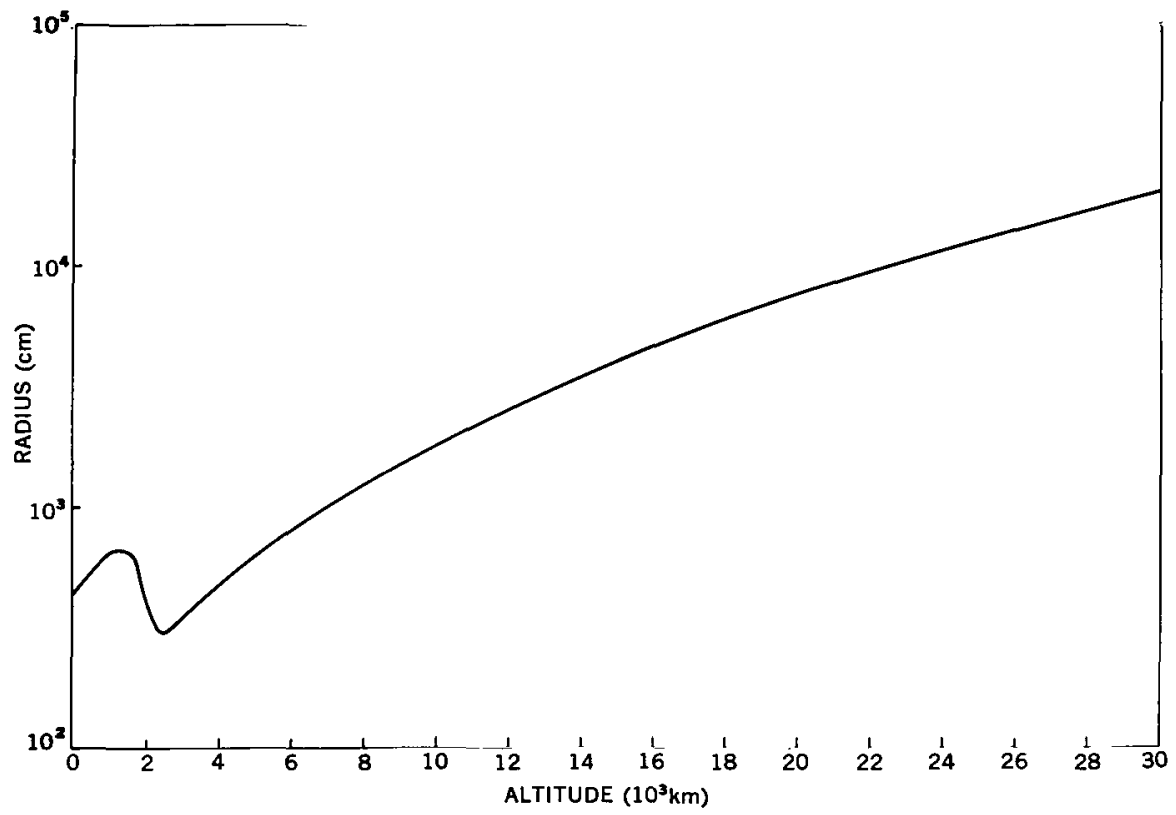

Figure 23 - Variation of the rms Larmor radius for positive ions in the exosphere

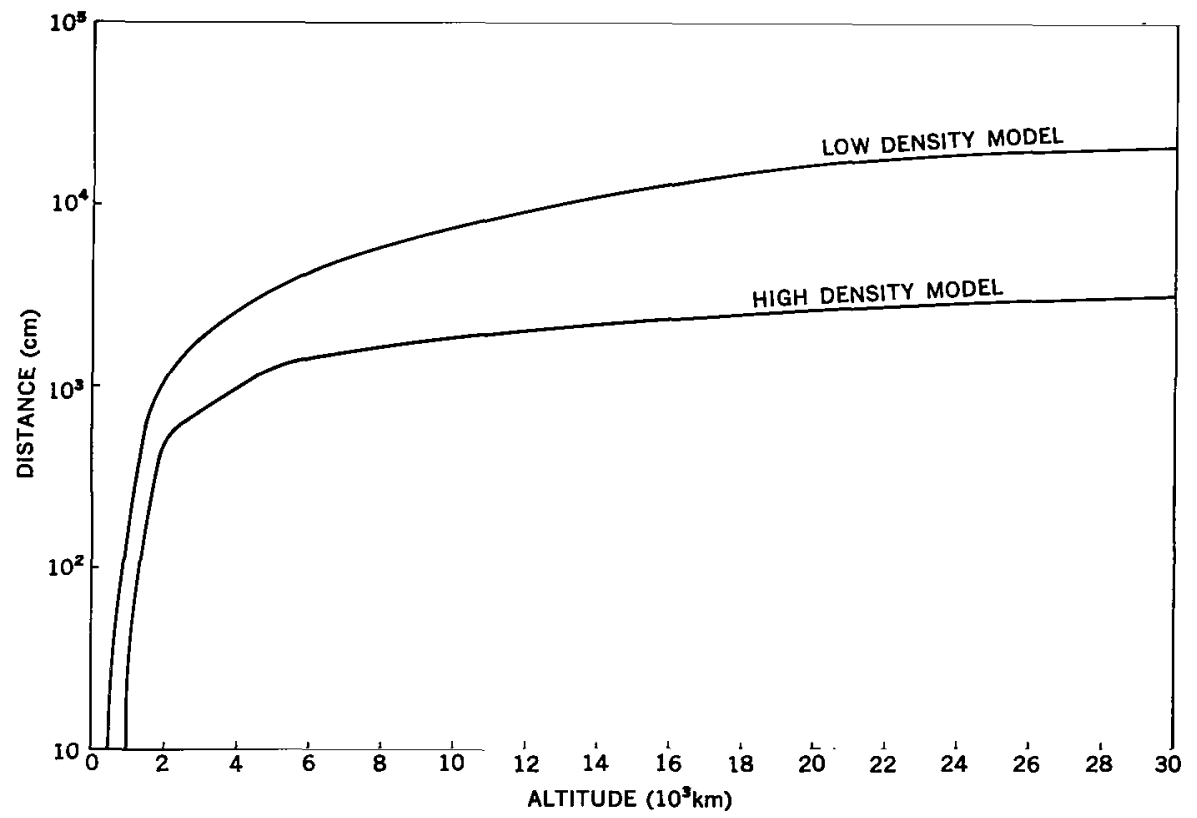

Figure 24 - Average distance a particle travels in exchanging 1 percent of its initial momentum shown for two models of the exosphere 
Figure 24 shows the approximate distance an electron travels in exchanging one percent of its initial momentum. The mean free path is then $10^{4}$ times the listed values. We note that in the outer exosphere of the high density model, a charged particle travels about one kilometer before exchanging 10 percent of its momentum; in the lower exosphere, it exchanges 10 percent in a distance on the order of meters.

The interpretation of Figures 23 and 24 is that for disturbances having a large length scale as compared to the distances plotted, the continuum equations yield at least a qualitative description. At distances that are small compared to those given, the problem should be treated either from the point of view of a self-consistent field theory or from a kinetic theory. It should be noted that the $\mathrm{T}$ and $\mathrm{V}$ modes, but not the $\mathrm{P}$ mode, might be excited by disturbances having an intermediate length scale.

The applicability of the macroscopic equations has been dealt with in terms of length rather than frequency collision time. The characteristic length can be converted into a characteristic frequency collision time provided that the disturbance has a characteristic macroscopic velocity.

In summary, the propagation of the $\mathrm{T}$ and $\mathrm{V}$ modes can be treated by the continuum equations at least qualitatively, provided that the disturbance has a long length scale as compared to some hundreds of meters in the outer exosphere and some tens of centimeters in the lower exosphere. The $\mathrm{P}$ mode propagation will be qualitatively described by the continuum approximation, provided that the length scale is large as compared to tens of meters in the lower exosphere and tens of kilometers in the upper exosphere. 


\section{PART V \\ DESCRIPTION OF SOURCES}

The gravitationally induced inhomogeneity of the charged particle density field does not enter into the description of a source, provided that the length scale associated with the source is small as compared to the length scale of the inhomogeneities. Additional problems arise in a layered medium in that the inhomogeneities may lead to mode conversion. In the present section we will consider the description of sources in a homogeneous medium.

The sources of the $\mathbf{P}$ and $\mathrm{T}$ modes differ in an important way from a source generating $\mathrm{V}$ waves: The source for the $\mathrm{V}$ mode must generate vorticity, while a pressure fluctuation is propagated in the $\mathrm{P}$ and $\mathrm{T}$ modes. A pressure fluctuation may arise from either the local injection of charged particles or heat. An example, in the exosphere, of such a pressure source would be the influx of ionizing radiation into a neutral gas, or the motion of a satellite or missile through the charged particle gas.

The region of allowed propagation of the $\mathrm{V}$ mode in the diagnostic diagram is confined to a line. This implies that the waves generated by a source characterized by a limited range of wave numbers will have a narrow band spectrum. The allowed region of the $T$ mode permits a broad spectrum. The $P$ wave will be intermediate, though at small values of $\beta$ the allowed region is nearly a line. The character of the spectrum of a magnetic disturbance can thus be used for a qualitative identification of the $\mathrm{V}$ and $\mathrm{T}$ modes.

A pressure source can be described in terms of some characteristic length $\mathrm{L}$. A source with a characteristic velocity $v$ has a characteristic frequency $V / L$. A qualitative picture of the proportion of $\mathrm{T}$ and $\mathrm{P}$ modes generated by a pressure source having a velocity $v$ and a length $L$ can be obtained from a consideration of the diagnostic diagram in Figure 1.

At a given frequency, a source with a small length scale (high characteristic wave number $\mathrm{k}$ ) will tend to have the energy concentrated near to the region of $\mathrm{P}$ mode propagation and, as a result, the source generates primarily $P$ waves. At the same frequency a source with a larger length scale will generate primarily $\mathrm{T}$ waves. Qualitatively, the characteristic length of the source should be compared with the characteristic wavelength of the mode at a given frequency. This wavelength is of the order $(\mathrm{L} / \mathrm{V}) \mathrm{C}_{0}$ and $(\mathrm{L} / \mathrm{V}) \mathrm{C}_{\mathrm{A}}$ for the $\mathrm{P}$ and $\mathrm{T}$ modes, respectively. Thus, for a satellite having a length scale of a meter and traveling at $8 \mathrm{~km} / \mathrm{sec}$, the characteristic wavelength of the $P$ mode is 
about 10 centimeters, while that of the $T$ mode is 300 meters. The satellite under these conditions would generate primarily $\mathrm{P}$ modes, provided that the continuum conditions were met.

The proportion of $\mathrm{V}$ waves that are generated by a given source depends on the strength of the vorticity source. Vorticity is generated by the breakdown of surfaces of discontinuity in the fluid flow. Surfaces of discontinuity can arise between fluids traveling at different velocities or when the fluid passes over a solid. The characteristic aspect of vorticity generation is that one part of the interior of the fluid is subjected to pressure for a time while the adjacent parts are not. Surfaces of discontinuity may arise at the boundary of the pressure pulse generated by incoming ionizing radiation or by the breakdown of the finite amplitude front associated with the plasma reaching the earth's field. In the case of satellites and missiles, vorticity is generated by the exhaust in a region of partially ionized gas. The dimensions of a typical rocket suggest that a $\mathrm{V}$ wave is generated at a peak frequency of about $10 \mathrm{cps}$, if the burnout occurs in or above the ionosphere. The resulting signal would not undergo geometric attenuation, but at high frequencies conductivity losses are large. An experimental verification is possible by observation at the conjugate point to the launch site.

Vorticity may also be generated in the wake of a satellite. The character of this wake is determined by the Reynolds number

$$
\mathrm{R}=\frac{\mathrm{VL}}{\nu} \approx \frac{\mathrm{VL}}{\mathrm{C}_{0} \mathrm{~L}_{\mathrm{M}}}
$$

where $\nu$ is the kinematic viscosity and $L_{M}$ is the mean free path. If the Reynolds number is much greater than one, the wake is turbulent (Reference 35). In the lower exosphere the Reynolds number is much less than one and the wake is laminar, with vorticity diffusing to form a broad disturbed region.

The validity of the foregoing comments regarding the effectiveness of a satellite as a source of magnetohydrodynamic waves depends on whether or not the medium can be treated as a continuum for disturbances having a length scale of the dimensions of a satellite. At 300 kilometers, the rms Larmor radius for the positive ions is of the order of a meter and a particle must travel about 3 meters in exchanging 10 percent of its momentum. Under these conditions we expect that a satellite having dimensions on the order of meters generates microscopic $\mathrm{V}$ and $\mathrm{T}$ waves, but that a continuum treatment of the $\mathbf{P}$ mode is only approximate.

A quantitative theory of the relative proportions of $\mathrm{P}, \mathrm{V}$, and $\mathrm{T}$ waves generated by a given source depends on the detailed treatment of finite amplitude phenomena. For a weak source, an estimate of the relative proportions of $P$ and $T$ modes is possible 
(Reference 27). We consider a pressure source $P_{s}$,

$$
P_{S}\left(x_{i}, t\right)=\frac{\partial p}{\partial t}+\rho_{0} C_{0}^{2}\left(\frac{\partial v_{1}}{\partial x_{1}}+\frac{\partial v_{2}}{\partial x_{2}}+\frac{\partial v_{3}}{\partial x_{3}}\right)
$$

which has the units of dynes $/ \mathrm{cm}^{2}$ per second. This pressure source is related to a mass source and to the net accession of heat by

$$
P_{S}=\left[\frac{\rho_{0}\left(\gamma_{0}-1\right)}{a_{0} T_{0}}\right] \mathrm{q}+\mathrm{C}_{0}{ }^{2} \mathrm{~S},
$$

where $q$ has units of ergs/gm per second and $S$ has the units of gram $/ \mathrm{cm}^{3}$ per second. The pressure source $\mathrm{P}_{\mathrm{S}}$ will not generate vorticity.

Eliminating $v_{i}$ and $p$ from the field equations (Equations 14 through 17) we obtain

$$
\begin{aligned}
\frac{\partial^{2} \mathrm{R}}{\partial \mathrm{t}^{2}}-\mathrm{C}_{0}^{2}\left[\frac{\partial^{2} \mathrm{R}}{\partial \mathrm{x}_{1}^{2}}+\frac{\partial^{2} \mathrm{R}}{\partial \mathrm{x}_{2}{ }^{2}}+\frac{\partial}{\partial \mathrm{x}_{3}}\left(\frac{\partial^{2}}{\partial \mathrm{x}_{1}^{2}}+\frac{\partial^{2}}{\partial \mathrm{x}_{2}^{2}}\right)\right] \mathrm{V}_{3}-\mathrm{C}_{\mathrm{A}}^{2} \mathrm{v}^{2} \mathrm{R} & =\frac{1}{\rho_{0}}\left(\frac{\partial^{2}}{\partial \mathrm{x}_{1}^{2}}+\frac{\partial^{2}}{\partial \mathrm{x}_{2}^{2}}\right) \mathbf{P}_{\mathrm{S}} \\
& \frac{\partial^{2} \mathrm{v}_{3}}{\partial \mathrm{t}^{2}}-\mathrm{C}_{0}^{2}\left[\frac{\partial \mathrm{R}}{\partial \mathrm{x}_{3}}+\frac{\partial^{2} \mathrm{v}_{3}}{\partial \mathrm{x}_{3}{ }^{2}}\right]=-\frac{1}{\rho_{0}} \frac{\partial \mathrm{P}_{\mathrm{S}}}{\partial \mathrm{x}_{3}},
\end{aligned}
$$

where $R$ is the two-dimensional divergence

$$
R=\frac{\partial v_{1}}{\partial x_{1}}+\frac{\partial v_{2}}{\partial x_{2}}
$$

Equations 35 and 36 combine to yield

$$
\begin{gathered}
\frac{\partial^{2}}{\partial \mathrm{t}^{2}}\left[\frac{\partial^{2}}{\partial \mathrm{t}^{2}}-\mathrm{C}_{0}^{2}+\mathrm{C}_{\mathrm{A}}^{2} \mathrm{~V}^{2}\right] \mathrm{R}+\mathrm{C}_{0}^{2} \mathrm{C}_{\mathrm{A}}^{2}\left(\frac{\partial^{2}}{\partial \mathrm{x}_{3}^{2}}\right) \mathrm{V}^{2} \mathrm{R}=-\rho_{0}\left(\frac{\partial^{2}}{\partial \mathrm{t}^{2}}\right)\left(\frac{\partial^{2}}{\partial \mathrm{x}_{1}{ }^{2}}+\frac{\partial^{2}}{\partial \mathrm{x}_{2}^{2}}\right) \mathrm{P}_{\mathrm{S}}, \\
\frac{\partial^{2}}{\partial \mathrm{t}^{2}}\left[\frac{\partial^{2}}{\partial \mathrm{t}^{2}}-\mathrm{C}_{0}^{2}+\mathrm{C}_{\mathrm{A}}^{2} \mathrm{~V}^{2}\right] \frac{\partial \mathrm{v}_{3}}{\partial \mathrm{x}_{3}}+\mathrm{C}_{0}{ }^{2} \mathrm{C}_{\mathrm{A}}{ }^{2} \mathrm{~V}^{2}\left(\frac{\partial^{3}}{\partial \mathrm{x}_{3}{ }^{3}}\right) \mathrm{v}_{3}=-\frac{1}{\rho_{0}}\left(\frac{\partial^{2}}{\partial \mathrm{t}^{2}}-\mathrm{C}_{\mathrm{A}}^{2} \mathrm{~V}^{2}\right)\left(\frac{\partial^{2}}{\partial \mathrm{x}_{3}^{2}}\right) \mathrm{P}_{\mathrm{S}} .
\end{gathered}
$$

The space-time Fourier transform of the source term for the two-dimensional divergence is then

$$
-\frac{1}{\rho_{0}} \omega^{2}\left(k^{2}-k_{3}^{2}\right) \text { F.T. }\left(P_{S}\right)
$$


and the corresponding Fourier transform of the source for $\frac{\partial v_{3}}{\partial x_{3}}$ is

$$
-\frac{1}{\rho_{0}}\left(\omega^{2}-C_{A}^{2} k^{2}\right) k_{3}^{2} \text { F.T. }\left(P_{S}\right)
$$

The source terms can also be written in terms of the net accession of mass and heat by using Equation 34.

A point in space and time has a constant Fourier transform of $P_{s}$, all wave numbers and all frequencies being equally represented in the source. A source that is a point source in time but is isotropic in space with a length scale $\mathrm{L}$ may be represented by

$$
\text { F.T. }\left(P_{S}\right)=\frac{1}{8 \pi^{3}} \exp \left(-\frac{k^{2} L^{2}}{4}\right)
$$

corresponding to a Gaussian pressure field distribution about the origin. A source with the characteristic frequency $\omega$ would include the $\delta$ function at that frequency.

The source terms (Equations 37 and 38) make their greatest contribution to a wave at those values of wave number and frequency that lie on the slowness surface. The slowness surface for the $T$ mode is approximately $\omega=C_{A} k$, and the source term for the $P$ mode (Equation 38 ) vanishes. Along the slowness surface for the $\mathbf{P}$ mode

$$
\mathrm{k}_{3}^{2} \approx \omega^{2} \mathrm{C}_{0}^{-2}\left(1+\beta^{2}\right)
$$

so that the greatest contributions $\mathrm{f}$ : om the source term of the $\mathbf{P}$ mode result when

$$
\frac{\mathrm{C}_{\mathrm{A}}^{2} \mathrm{k}^{2}}{\omega^{2}}>>1
$$

Since $k^{2} e^{-k^{2} L^{2} / 4}$ has a maximum at $k=(2 L)^{-1}$ the greatest contribution of the source term to the $\mathbf{P}$ mode is for

$$
\frac{\mathrm{C}_{\mathrm{A}}^{2}}{\omega^{2} \mathrm{~L}^{2}}>1
$$

Small sources at low frequencies thus contribute most heavily to the $P$ mode. The contribution of the source (Equation 37) to the $T$ mode at a fixed value of $k_{3}$, is greatest for $k=(2 L)^{-1}$ and at high frequencies. 
The foregoing representation of the source is, in a sense, conventional and applicable only if the discontinuity in pressure is not too great. For a large discontinuity, the source region will be turbulent. Turbulent interaction will lead to a complex source function. In addition to the direct generation of $\mathbf{P}$ and $\mathbf{T}$ modes by the action of pressure or entropy sources, vorticity can generate the $\mathrm{P}$ mode, as can temperature irregularities.

As an example of some of the difficulties introduced in treating strong sources, we consider the Argus experiments (References 36 and 37). In these experiments approximately $10^{19}$ ergs of energy were released at a height of about 450 kilometers. The time behavior of the source is well represented by a $\delta$ function, but there is no unique length scale. We might, for instance, consider the length as determined by the distance over which the initial pressure pulse must spread in order to attain the ambient pressure. The initial pressure of the charged particles is on the order of $10^{-7}$ dynes $/ \mathrm{cm}^{2}$, so that the indicated length scale would be of the order of 1000 kilometers. This length is large compared to the variation in charged particle density, so that the source cannot be considered isotropic.

A source with a length scale of 1000 kilometers would generate $T$ waves with a peak frequency of a few tenths of a cycle per second. The observed first pulse appears to have been peaked at around one cps (Reference 37). In addition, the source should have generated low frequency $P$ waves; but no attempt was made to detect such a mode. The turbulence associated with the outgoing shock should have generated vorticity leading to $\mathrm{V}$ wave propagation, and there is some indication that these waves were excited: a sinusoidal waveform at about one cycle per second was observed at the conjugate point to the explosion.

Berthold, Harris and Hope (Reference 38) find that the amplitude of the low frequency magnetic signal generated by Argus was greatest along the geomagnetic meridian. The signal strength fell off rapidly as the direction of propagation deviated from the geomagnetic meridian. Such an amplitude pattern is consistent with $\mathrm{V}$ mode propagation without geometrical attenuation along the line of force into the ionosphere. The propagation in the atmosphere was then a radially spreading electromagnetic wave, badly disturbed by the partially conducting lower and upper boundaries of the atmosphere. 


\section{PART VI \\ SPECTRUM OF MAGNETIC DISTURBANCES}

The spectrum of a hydromagnetic disturbance will be determined primarily by the source characteristics. The high frequency cutoff is fixed by the Larmor radius at the source (Reference 8). In the lower exosphere the Larmor radius is of the order of meters, and the corresponding cutoff frequency for the $\mathrm{V}$ and $\mathrm{T}$ modes is on the order of 100 cycles per second. Higher frequency waves will be propagated as modified electromagnetic waves. In the outer exosphere the Larmor radius is on the order of $10^{4}$ centimeters, and the corresponding cutoff frequency is on the order of a few cps. Thus, the high frequency cutoff of a magnetic disturbance can serve as an indication of source location. The ground level distinction between waves that have propagated as electromagnetic waves from source to receiver and those that have traveled as hydromagnetic waves to the base of the ionosphere and then as electromagnetic waves can be made on the basis of travel times. The source dimensions and time scale will determine the character of the initial onset: A source with a large length scale will principally generate the high frequency $\mathrm{T}$ mode, and some low frequency $\mathrm{P}$ mode; and the proportion of $\mathrm{V}$ mode will depend on the sharpness of the discontinuity. The $V$ mode may be identified by its characteristic narrow band spectrum.

At low frequencies it is possible that hydromagnetic modes modified by gravity become important. The characteristic gravitational frequency $N_{1}$ as a function of altitude is shown in Figure 25. In the lower exosphere this characteristic frequency has a period of the order of 10 minutes, while in the upper exosphere it is on the order of 15 hours. For frequencies that are large compared to this characteristic frequency, the propagation is in the unperturbed $\mathrm{P}, \mathrm{T}$, and $\mathrm{V}$ modes. There will be a gap in the spectrum at those frequencies separating the gravitational modes from the transverse modes. (See Figures 5, 13, 14, and 15).

The presence of the boundaries in the system opens the possibility of trapping of the energy in a given mode. At the lower boundary of the exosphere, there is a rapid variation in the charged particle density. However, there is no evidence for the existence of an upper boundary; the charged particle distribution grades into the interplanetary or coronal density. It thus appears unlikely that the $T$ mode can be trapped in a resonant cavity formed by the lower boundary of the exosphere and the disordered outer regions where the earth's magnetic field ends and the interplanetary field begins. 
52

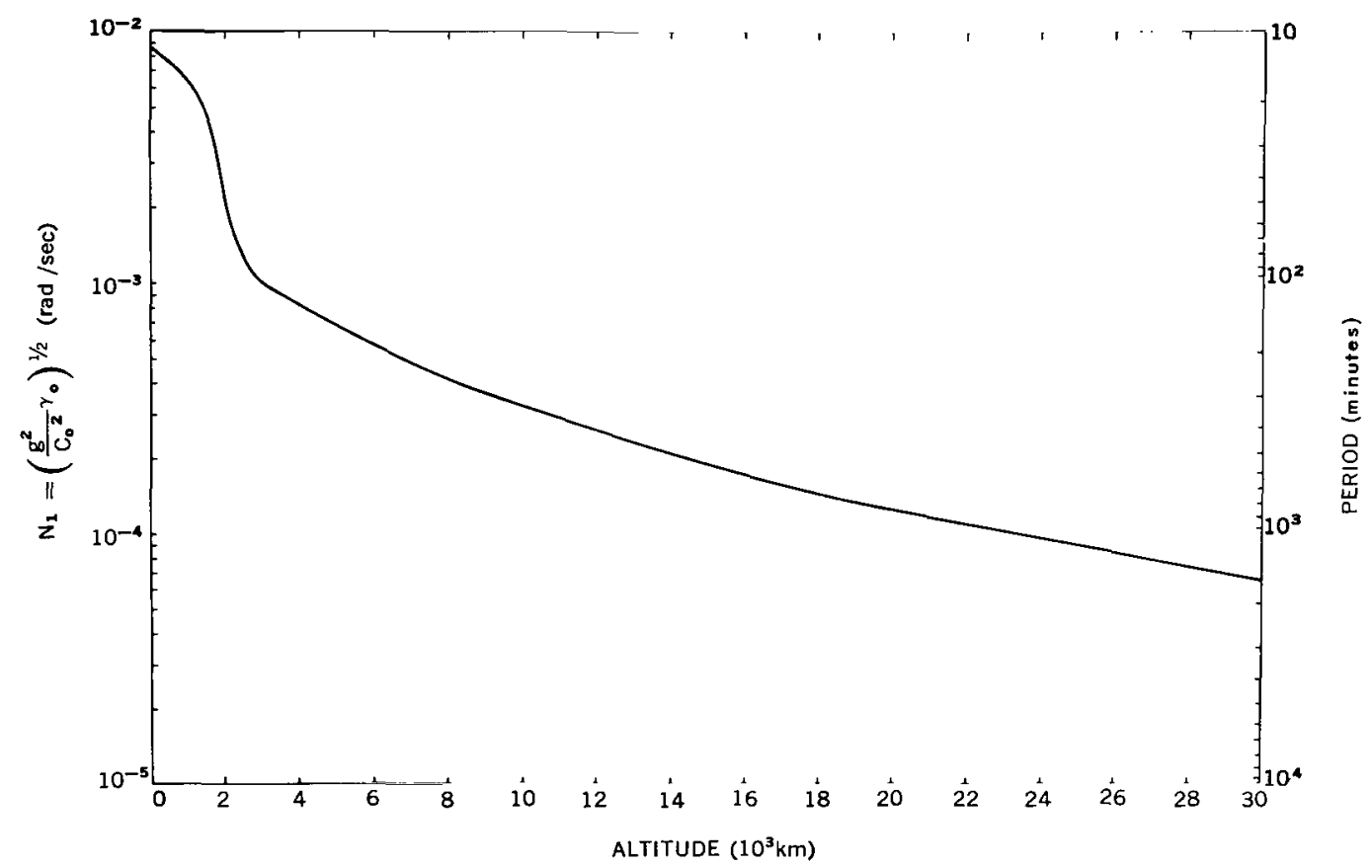

Figure 25 - Variation of the critical frequency $N_{1}$ as a function of altitude

The $\mathbf{P}$ mode will be propagated one-dimensionally along the lines of force, since $\beta$ is on the order of $10^{-2}$ to $10^{-3}$ over the entire exosphere. The propagation is similar to the propagation in a pipe with a bulge in the middle. Because of the density contrast in the $F 2$ regions, the $\mathrm{P}$ modes can be trapped along the line of force and it is possible that a free oscillation or standing wave could be set up. The period of such a standing wave would be on the order of several hours to a day. These long period vibrations require a consideration of the rotational effects, particularly the Coriolis force, and these have not been included in the foregoing formulation. In addition, viscosity is of considerable importance since it varies as the mean free path, and the latter differs by a factor of $10^{3}$ between the lower and upper exosphere. Thus the possibility exists that a free oscillation in the $\mathbf{P}$ mode may be set up, though rotational and viscous effects will be of great importance.

The $\mathrm{V}$ mode propagates vorticity along the line of force. The rapid variation of charged particle density at both ends of the line of force suggests that energy could be partially trapped, leading to a free oscillation in the $\mathrm{V}$ mode. Since the charged particle density varies continuously, the mode will be a leaky mode with energy passing through and radiating out as an electromagnetic wave at the end of the line of force. A rough estimate is obtained by taking the wave impedance as proportional to $\rho^{1 / 2}$. The density contrast in the ionosphere then suggests a $Q$ for this mode of oscillation on the order of 10, all internal losses being neglected. 
A direct calculation of the free period of this mode of oscillation is straightforward. The equation for the propagation of vorticity

$$
\frac{\partial^{2} \xi}{\partial t^{2}}-C_{A}^{2}(x)\left(\frac{\partial^{2} \xi}{\partial x^{2}}\right)=0,
$$

where $\mathrm{x}$ is the distance along the line of force, is a one-dimensional wave equation with a variable phase velocity. The boundary conditions depend on the details of the physical process at the two ends of the lines of force. If the proportion of energy leaking out of the mode is small, then the boundary condition is that the vorticity vanishes. The altitude at which vorticity vanishes is fixed by the condition that the charged-neutral particle collision frequency be high compared to the Larmor frequency. It should be noted that the vanishing of the vorticity implies the vanishing of a current along the line of force. Under these conditions, the eigen frequencies of a uniform system are $f_{n}=n_{A} / 2 L$, where $\mathrm{L}$ is the length of the line of force and $\mathrm{n}$ is the number of the overtone.

Calculations have been carried out to determine the free periods for $\mathrm{V}$ mode oscillations for a variable $\mathrm{C}_{\mathrm{A}}$ amploying the two models of the exosphere. The results are shown in Tables 1 and 2 where the periods are listed as a function of geomagnetic latitude. These calculations do not take into account the possible leaking of energy out the end of the line of force.

The sharp maximum in the Alfvén wave velocity at altitudes of about $3000 \mathrm{~km}$ (see Figure 22) may allow the trapping of energy between this maximum and the F2 layer. The characteristic periods (Tables 3 and 4) are shorter than the periods of oscillation along the whole length of the line of force.

Table 1

Free Periods of Oscillation in the V Mode for the High Density Model

\begin{tabular}{|c|c|c|c|c|}
$\begin{array}{c}\text { Geomagnetic } \\
\text { Latitude }\end{array}$ & \multicolumn{4}{|c|}{ Period (seconds) } \\
(degrees) & $\mathrm{n}=1$ & $\mathrm{n}=2$ & $\mathrm{n}=3$ & $\mathrm{n}=4$ \\
\hline 30 & 14.2 & 12.8 & 6.17 & 5.65 \\
35 & 12.0 & 11.1 & 5.47 & 4.85 \\
40 & 11.0 & 9.98 & 7.01 & 4.35 \\
45 & 14.5 & 9.16 & 8.69 & 5.39 \\
50 & 29.1 & 11.0 & 8.46 & 8.12 \\
55 & 67.3 & 24.3 & 15.3 & 11.2 \\
60 & 184.6 & 64.8 & 40.2 & 29.4 \\
& & & &
\end{tabular}


Table 2

Free Periods of Oscillation in the V Mode for the Low Density Model

\begin{tabular}{|c|c|c|c|c|}
\hline $\begin{array}{c}\text { Geomagnetic } \\
\text { Latitude } \\
\text { (degrees) }\end{array}$ & \multicolumn{4}{|c|}{ Period (seconds) } \\
\cline { 2 - 5 } & $\mathrm{n}=1$ & $\mathrm{n}=2$ & $\mathrm{n}=3$ & $\mathrm{n}=4$ \\
\hline 30 & 12.1 & 10.3 & 4.85 & 4.39 \\
35 & 9.98 & 9.01 & 3.99 & 3.69 \\
40 & 8.77 & 8.04 & 4.11 & 3.24 \\
45 & 8.63 & 7.34 & 6.00 & 3.09 \\
50 & 13.9 & 6.95 & 6.59 & 4.98 \\
55 & 29.2 & 10.9 & 7.10 & 6.28 \\
60 & 74.4 & 26.4 & 16.6 & 12.1 \\
\hline
\end{tabular}

Table 3

Periods of Oscillation for Energy Trapped Between F2 and Maximum in the $\mathrm{C}_{\mathrm{A}}$ Curve for the High Density Model

\begin{tabular}{|c|c|c|c|c|}
\hline \multirow{2}{*}{$\begin{array}{c}\text { Geomagnetic } \\
\text { Latitude } \\
\text { (degrees) }\end{array}$} & \multicolumn{4}{|c|}{ Period (seconds) } \\
\cline { 2 - 5 } & $\mathrm{n}=1$ & $\mathrm{n}=2$ & $\mathrm{n}=3$ & $\mathrm{n}=4$ \\
\hline 35 & 10.8 & 4.75 & 3.09 & 2.30 \\
40 & 9.38 & 4.11 & 2.67 & 1.98 \\
45 & 8.42 & 3.67 & 2.38 & 1.77 \\
50 & 7.68 & 3.35 & 2.17 & 1.61 \\
55 & 7.14 & 3.11 & 2.01 & 1.50 \\
60 & 6.73 & 2.93 & 1.89 & 1.41 \\
\hline
\end{tabular}

Table 4

Periods of Oscillation for Energy Trapped Between F2 and Maximum in the $\mathrm{C}_{\mathrm{A}}$ Curve for the Low Density Model

\begin{tabular}{|c|c|c|c|c|}
\hline $\begin{array}{c}\text { Geomagnetic } \\
\text { Latitude } \\
\text { (degrees) }\end{array}$ & \multicolumn{4}{|c|}{ Period (seconds) } \\
\cline { 2 - 5 } & $\mathrm{n}=1$ & $\mathrm{n}=2$ & $\mathrm{n}=3$ & $\mathrm{n}=4$ \\
\hline 40 & 7.53 & 3.10 & 1.98 & 1.46 \\
45 & 6.66 & 2.75 & 1.75 & 1.29 \\
50 & 6.05 & 2.49 & 1.59 & 1.17 \\
55 & 5.65 & 2.31 & 1.47 & 1.08 \\
60 & 5.26 & 2.16 & 1.38 & 1.02 \\
\hline
\end{tabular}


A weak source located below the 3000 kilometer maximum would primarily excite the shorter period oscillations. Disturbances in the upper ionosphere associated with day-night effects may provide such a source. A sufficiently strong source in the upper exosphere would be expected to excite both the short and long period oscillations.

A detailed comparison between the computed and observed periods is difficult because the data presented, for example, by Benioff (Reference 14) and Troitskaya (Reference 16) are in the form of analog records. The obvious complexity of the spectra precludes a zero-crossing analysis. The study of Tepley (Reference 39) shows micropulsations with a frequency of 5 to 0.5 cycles per second, together with higher frequencies. These frequencies are higher than would be expected for a free mode oscillation at the geomagnetic latitude of Palo Alto. 


\section{ACKNOWLEDGMENTS}

The author wishes to thank Clarence Wade for aid in numerically evaluating the numerous polynomials, and William Cahill and Dwight Sumida for carrying out the calculations of the free periods of the $V$ mode.

\section{REFERENCES}

1. Chapman, S., and Ferraro, V. C. A., "A New Theory of Magnetic Storms, Part IThe Initial Phase," Terrestrial Magnetism and Atmospheric Electricity 36(2):77-97, June 1931; (Part I continued) 36(3):171-186, September 1931

2. Chapman, S., and Ferraro, V. C. A., "A Theory of Magnetic Storms, Part II - The Main Phase," Terrestrial Magnetism and Atmospheric Electricity 38(2):79-96, June 1933

3. Chapman, S., and Ferraro, V. C. A., "The Theory of the First Phase of a Geomagnetic Storm," Terrestrial Magnetism and Atmospheric Electricity 45(3):245-268, September 1940

4. Storey, L. R. O., "An Investigation of Whistling Atmospherics," Phil. Trans. Roy. Soc. London 246A(908):113-141, 1953

5. Behr, A., and Siedentopf, H., "Untersuchungen über Zodiakallicht und Gegenschein nach lichtelektrischen Messungen auf dem Jungfraujoch," Zeit. für Astrophys. $32(1): 19-50,1953$

6. Blackwell, D. E., and Ingham, M. F., "Observations of the Zodiacal Light from a Very High Altitude Station. II. Electron Densities in Interplanetary Space," Mon. Not. Roy. Astronom. Soc. 122(2):129-141, 1961

7. Dessler, A. J., "The Propagation Velocity of World-Wide Sudden Commencements of Magnetic Storms," J. Geophys. Res. 63(2):405-408, June 1958

8. Dessler, A. J., and Parker, E. N., "Hydromagnetic Theory of Geomagnetic Storms," J. Geophys. Res. 64(12):2239-2252, December 1959

9. Piddington, J. H., "The Transmission of Geomagnetic Disturbances Through the Atmosphere and Interplanetary Space," Geophys. J. 2(3):173-189, September 1959

10. Piddington, J. H., "Geomagnetic Storm Theory," J. Geophys. Res. 65(1):93-106, January 1960

11. Piddington, J. H., "A Theory of Polar Geomagnetic Storms," Geophys. J. 3(3):314332, September 1960 
12. Dessler, A. J., Francis, W. E., and Parker, E. N., "Geomagnetic Storm SuddenCommencement Rise Times," J. Geophys. Res. 65(9):2715-2719, September 1960

13. Williams, V. L., "The Simultaneity of Sudden Commencements of Magnetic Storms," J. Geophys. Res. 65(1):85-92, January 1960

14. Benioff, H., "Observations of Geomagnetic Fluctuations in the Period Range 0.3 to 120 Seconds," J. Geophys. Res. 65(5):1413-1422, May 1960

15. Jacobs, J. A., and Sinno, K., "World-Wide Characteristics of Geomagnetic Micropulsations," Geophys. J. 3(3):333-353, September 1960

16. Troitskaya, V. A., "Pulsation of the Earth's Electromagnetic Field with Periods of 1-15 Seconds and Their Connection with Phenomena in the High Atmosphere," J. Geophys. Res. 66(1):5-18, January 1961

17. Herlofson, N., "Magneto-Hydrodynamic Waves in a Compressible Fluid Conductor," Nature 165(4208):1020-1021, June 24, 1950

18. Friedrichs, K. O., and Kranzer, H., "Notes on Magneto-Hydrodynamics. VIII. Nonlinear Wave Motion," New York University, Institute of Mathematical Sciences, AEC Computing and Applied Mathematics Center, NYO-6486, July 31, 1958

19. Bazer, J., and Fleischman, O., "Propagation of Weak Hydromagnetic Discontinuities," Physics of Fluids 2(4):366-378, July-August 1959

20. Grad, H., "Propagation of Magnetohydrodynamic Waves Without Radial Attenuation," in: The Magnetodynamics of Conducting Fluids ed. by D. Bershader, Stanford: Stanford University Press, 1959, pp. 37-60.

21. Eckart, C., "Hydrodynamics of Oceans and Atmospheres," Oxford: Pergamon Press, 1960

22. Eckart, C., "The Approximate Solution of One-Dimensional Wave Equations," Rev. Modern Phys. 20(2):399-417, April 1948

23. Whitman, G. B., "A Note on Group Velocity," J. Fluid Mechanics Vol. 9, Part III, pp. 347-352, November 1960

24. Synge, J. L., "Elastic Waves in Anisotropic Media," J. Math. and Phys. 35(4):323-334, January 1957

25. Weatherburn, C. E., "Differential Geometry of Three Dimensions," Cambridge: Cambridge University Press, 1927

26. Eisenhart, L. P., "An Introduction to Differential Geometry," Princeton: Princeton University Press, 1940

27. Lighthill, M. J., "Studies on Magneto-Hydrodynamic Waves and Other Anisotropic Wave Motions," Phil. Trans. Roy. Soc. London 252A:397-430, March 31, 1960 
28. Tanwal, R. P., and Truesdell, C., "Electric Current and Fluid Spin Created by the Passage of a Magnetosonic Wave," Archives for Rational Mechanics and Analysis $5(5) ; 432-439,1960$

29. Carstoiu, J., "Hydromagnetic Waves in a Compressible Fluid Conductor," Proceedings of the National Academy of Sciences of the U. S. A. 46(1):131-136, January 1960

30. Ferraro, V. C. A., "The Mean Free Path in Rare Ionized Gases," Mon. Not. Roy. Astronom. Soc. 93(6):416-422, April 1933

31. Spitzer, L., "Physics of Fully Ionized Gases," New York: Interscience Publishers, 1956

32. Johnson, F. S., "The Ion Distribution Above the $\mathrm{F}_{2}$ Maximum," J. Geophys. Res. 65(2):577-584, February 1960

33. Hanson, W. B., and McKibbin, D. D., "An Ion-Trap Measurement of the Ion Concentration Profile above the $\mathrm{F}_{2}$ Peak," J. Geophys. Res. 66(6):1667-1671, June 1961

34. Smith, R. L., and Helliwell, R. A., "Electron Densities to 5 Earth Radii Deduced from Nose Whistlers," J. Geophys. Res. 65(9):2583, September 1960

35. Townsend, A. A., "The Structure of Turbulent Shear Flow," Cambridge: University Press, 1956

36. Christofilos, N. C., "The Argus Experiment," J. Geophys. Res. 64(8):869-875, August 1959

37. Newman, P., "Optical, Electromagnetic, and Satellite Observations of High-Altitude Nuclear Detonations. I.," J. Geophys. Res. 64(8):923-932, August 1959

38. Berthold, W. K., Harris, A. K., and Hope, H. J., "World-Wide Effects of Hydromagnetic Waves Due to Argus," J. Geophys. Res. 65(8):2233-2239, August 1960

39. Tepley, L. R., "Observations of Hydromagnetic Emissions," J. Geophys. Res. 66(6):1651-1658, June 1961 